\title{
TASI 2011 lectures notes: two-component fermion notation and supersymmetry
}

\author{
Stephen P. Martin \\ Physics Department \\ Northern Illinois University \\ DeKalb IL 60115, USA
}

These notes, based on work with Herbi Dreiner and Howie Haber, discuss how to do practical calculations of cross sections and decay rates using two-component fermion notation, as appropriate for supersymmetry and other beyond-the-Standard-Model theories. Included are a list of two-component fermion Feynman rules for the Minimal Supersymmetric Standard Model, and some example calculations.

\section{Introduction}

2 Notations and conventions 2

2.1 Two-component spinors . . . . . . . . . . . . . . 2

2.2 Fermion interaction vertices . . . . . . . . . . . . 8

2.3 External wavefunctions for 2-component spinors . . . . . . . . . . 10

2.4 Propagators ........................... . . . . . . . . . . . . .

2.5 General structure and rules for Feynman graphs . . . . . . . . . . . . 12

2.6 Conventions for names and fields of fermions and antifermions . . . . . . 13

3 Feynman rules for fermions in the Standard Model 15

4 Fermion Feynman rules in the Minimal Supersymmetric Standard Model

5 Examples 25

5.1 Top quark decay: $t \rightarrow b W^{+} \ldots \ldots \ldots \ldots \ldots \ldots \ldots \ldots$

$5.2 Z^{0}$ vector boson decay: $Z^{0} \rightarrow f \bar{f} \ldots \ldots \ldots \ldots \ldots \ldots$

5.3 Bhabha scattering: $e^{-} e^{+} \rightarrow e^{-} e^{+} \ldots \ldots \ldots \ldots \ldots \ldots \ldots$

5.4 Neutral MSSM Higgs boson decays $\phi^{0} \rightarrow f \bar{f}$, for $\phi^{0}=h^{0}, H^{0}, A^{0} \ldots \ldots .30$

5.5 Neutralino decays $\widetilde{N}_{i} \rightarrow \phi^{0} \widetilde{N}_{j}$, for $\phi^{0}=h^{0}, H^{0}, A^{0} \ldots \ldots \ldots \ldots$

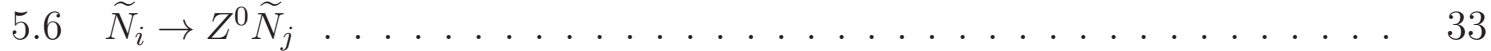

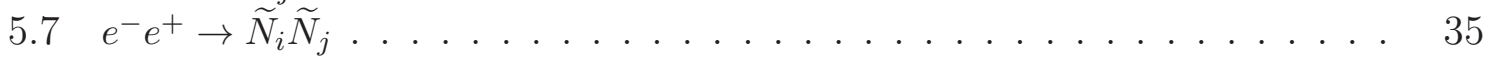

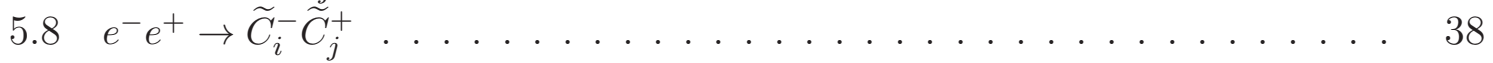

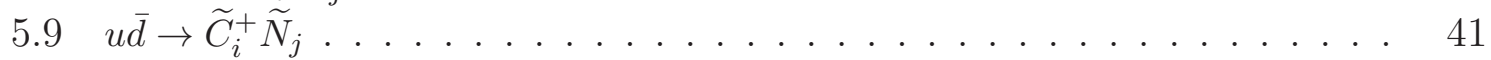

5.10 Neutralino decay to photon and Goldstino: $\widetilde{N}_{i} \rightarrow \gamma \widetilde{G} \ldots \ldots . \ldots . .43$

5.11 Gluino pair production from gluon fusion: $g g \rightarrow \widetilde{g} \widetilde{g} \ldots \ldots \ldots \ldots$

6 Conclusion 48

References 


\section{Introduction}

For nearly the past four decades, the imaginations of particle theorists have been running unchecked, producing many clever ideas for what might be beyond the Standard Model of particle physics. Now the LHC is confronting these ideas. The era of "clever" in model-building may soon come to an end, in favor of a new era where the emphasis is more on "true".

At TASI 2011 in Boulder, I talked about supersymmetry, which is probably the most popular class of new physics models. These are nominally the notes for those lectures. However, there are already many superb reviews on supersymmetry from diverse points of view [1]-[10]. Besides, everything I covered in Boulder is already presented in much more depth in my own attempt at an introduction to supersymmetry, A supersymmetry primer [11], which was updated just after TASI, in September 2011. That latest version includes a new section on superspace and superfields. I'm not clever enough to think of better ways to say the same things, so perhaps you could just download that and read it instead. Then, when you are done, I'll continue with a complementary topic that I could have talked about at TASI 2011, but didn't for lack of time. So please go ahead and read the Primer now, as a prerequisite for the following. Take your time, I'll wait.

Good, you're back!

One of the most fundamental observations about physics at the weak scale is that it is chiral; the left-handed and right-handed components of fermions are logically distinct objects that have different gauge transformation properties. Despite this, textbooks on quantum field theory usually present calculations, such as cross-sections, decay rates, anomalies, and self-energy corrections, in the 4-component fermion language. One often hears that even though 2-component fermion language is better for devising many theories, including supersymmetry, it is somehow not practical for real calculations of physical observables. Herbi Dreiner, Howie Haber, and I decided to confront this erroneous notion by working out a complete formalism for doing such practical calculations, treating Dirac, Majorana and Weyl fermions in a unified way. The result went into a rather voluminous report [12]. In the rest of these notes, I will try to introduce our formalism in a more concise form, leaving out derivations and giving just enough examples to illustrate the main ideas.

\section{Notations and conventions}

\subsection{Two-component spinors}

First, the terrible issue of the sign of the metric. I use the correct (mostly plus sign) metric. Herbi and Howie both use the wrong metric (the one with mostly minus signs), but they won a 2-1 vote on which to use in the journal and arXiv versions of ref. [12]. So far, the United States Supreme Court has declined to intervene on my behalf. However, we did devise a LaTeX macro to convert the sign of the metric, so there is an otherwise 
identical version of ref. [12, with my metric, which you can get from the web page linked to in its arXiv abstract page. Conversely, if you don't like my metric sign, you can download a version of these notes from a web page linked to in the comments section of the arXiv abstract page. You can tell which version you are presently reading from this:

$$
g_{\mu \nu}=g^{\mu \nu}=\operatorname{diag}(-1,+1,+1,+1) .
$$

Here $\mu, \nu=0,1,2,3$ are spacetime vector indices.

Contravariant four-vectors (e.g. positions and momenta) are defined with raised indices, and covariant four-vectors (e.g. derivatives) with lowered indices:

$$
\begin{aligned}
& x^{\mu}=(t ; \overrightarrow{\boldsymbol{x}}), \quad p^{\mu}=(E ; \overrightarrow{\boldsymbol{p}}), \\
& \partial_{\mu} \equiv \frac{\partial}{\partial x^{\mu}}=(\partial / \partial t ; \vec{\nabla}),
\end{aligned}
$$

in units with $c=1$. The totally antisymmetric pseudo-tensor $\epsilon^{\mu \nu \rho \sigma}$ is defined such that

$$
\epsilon^{0123}=-\epsilon_{0123}=+1 \text {. }
$$

Two-component fermions transform in either the $\left(\frac{1}{2}, 0\right)$ (left-handed) or $\left(0, \frac{1}{2}\right)$ (righthanded) spinor representations of the Lorentz group. By convention, the $\left(\frac{1}{2}, 0\right)$ rep carries an undotted spinor index $\alpha, \beta, \ldots$, and the $\left(0, \frac{1}{2}\right)$ rep carries a dotted index $\dot{\alpha}, \dot{\beta}, \ldots$, each running from 1 to 2. If $\psi_{\alpha}$ is a left-handed Weyl spinor, then the Hermitian conjugate

$$
\psi_{\dot{\alpha}}^{\dagger} \equiv\left(\psi_{\alpha}\right)^{\dagger}
$$

is a right-handed Weyl spinor. Therefore, any particular fermionic degrees of freedom can be described equally well using a left-handed Weyl spinor or a right-handed one. By convention, the names of spinors are chosen so that right-handed spinors always carry daggers, and left-handed spinors do not. The spinor indices are raised and lowered using the 2-component antisymmetric object

$$
\epsilon^{12}=-\epsilon^{21}=\epsilon_{21}=-\epsilon_{12}=1
$$

as follows:

$$
\psi_{\alpha}=\epsilon_{\alpha \beta} \psi^{\beta}, \quad \psi^{\alpha}=\epsilon^{\alpha \beta} \psi_{\beta}, \quad \psi_{\dot{\alpha}}^{\dagger}=\epsilon_{\dot{\alpha} \dot{\beta}} \psi^{\dagger \dot{\beta}}, \quad \psi^{\dagger \dot{\alpha}}=\epsilon^{\dot{\alpha} \dot{\beta}} \psi_{\dot{\beta}}^{\dagger},
$$

with repeated indices summed over, and

$$
\psi^{\dagger \dot{\alpha}} \equiv\left(\psi^{\alpha}\right)^{\dagger}
$$

When constructing Lorentz tensors from fermion fields, the heights of spinor indices must be consistent in the sense that lowered indices must only be contracted with raised indices.

To make contact with the (perhaps more familiar) 4-component language, a Dirac spinor consists of two independent 2-component Weyl spinors, united into a 4component object:

$$
\Psi_{D}=\left(\begin{array}{c}
\xi_{\alpha} \\
\chi^{\dagger \dot{\alpha}}
\end{array}\right)
$$


while a 4-component Majorana spinor has the same form, but with the two spinors identified through Hermitian conjugation:

$$
\Psi_{\mathrm{M}}=\left(\begin{array}{c}
\psi_{\alpha} \\
\psi^{\dagger \dot{\alpha}}
\end{array}\right)
$$

The free Dirac and Majorana Lagrangians are:

$$
\begin{aligned}
\mathcal{L}_{\text {Dirac }} & =i \bar{\Psi}_{D} \gamma^{\mu} \partial_{\mu} \Psi_{D}-m \bar{\Psi}_{D} \Psi_{D} ; \\
\mathcal{L}_{\text {Majorana }} & =\frac{i}{2} \bar{\Psi}_{M} \gamma^{\mu} \partial_{\mu} \Psi_{M}-\frac{1}{2} m \bar{\Psi}_{M} \Psi_{M},
\end{aligned}
$$

where

$$
\bar{\Psi}_{D}=\Psi_{D}^{\dagger}\left(\begin{array}{ll}
0 & 1 \\
1 & 0
\end{array}\right)=\left(\begin{array}{ll}
\chi^{\alpha} & \xi_{\dot{\alpha}}^{\dagger}
\end{array}\right),
$$

and similarly for the Majorana 4-component spinor:

$$
\bar{\Psi}_{M}=\Psi_{M}^{\dagger}\left(\begin{array}{ll}
0 & 1 \\
1 & 0
\end{array}\right)=\left(\begin{array}{ll}
\psi^{\alpha} & \psi_{\dot{\alpha}}^{\dagger}
\end{array}\right),
$$

and the $4 \times 4$ gamma matrices can be represented by

$$
\gamma^{\mu}=\left(\begin{array}{cc}
0 & \sigma^{\mu} \\
\bar{\sigma}^{\mu} & 0
\end{array}\right)
$$

where

$$
\begin{array}{ll}
\sigma^{0}=\bar{\sigma}^{0}=\left(\begin{array}{ll}
1 & 0 \\
0 & 1
\end{array}\right), & \sigma^{1}=-\bar{\sigma}^{1}=\left(\begin{array}{cc}
0 & 1 \\
1 & 0
\end{array}\right) \\
\sigma^{2}=-\bar{\sigma}^{2}=\left(\begin{array}{cc}
0 & -i \\
i & 0
\end{array}\right), & \sigma^{3}=-\bar{\sigma}^{3}=\left(\begin{array}{cc}
1 & 0 \\
0 & -1
\end{array}\right) .
\end{array}
$$

In order to include chiral interactions for these fermions in the 4-component language, one must define $P_{L}$ and $P_{R}$ projection operators:

$$
P_{L} \Psi_{D}=\left(\begin{array}{c}
\xi_{\alpha} \\
0
\end{array}\right), \quad P_{R} \Psi_{D}=\left(\begin{array}{c}
0 \\
\chi^{\dagger \dot{\alpha}}
\end{array}\right) .
$$

In the 2-component language, the Dirac Lagrangian is

$$
\mathcal{L}_{\text {Dirac }}=i \xi_{\dot{\alpha}}^{\dagger}\left(\bar{\sigma}^{\mu}\right)^{\dot{\alpha} \beta} \partial_{\mu} \xi_{\beta}+i \chi^{\alpha}\left(\sigma^{\mu}\right)_{\alpha \dot{\beta}} \partial_{\mu} \chi^{\dagger \dot{\beta}}-m\left(\xi_{\dot{\alpha}}^{\dagger} \chi^{\dagger \dot{\alpha}}+\chi^{\alpha} \xi_{\alpha}\right)
$$

This establishes that the sigma matrices $\left(\bar{\sigma}^{\mu}\right)^{\dot{\alpha} \beta}$ and $\left(\sigma^{\mu}\right)_{\alpha \dot{\beta}}$ have the spinor indices with heights as indicated. It is traditional and very convenient to suppress these indices wherever possible using the convention that descending contracted undotted indices and ascending contracted dotted indices,

$$
\alpha_{\alpha} \quad \text { and } \quad \dot{\alpha},
$$

can be omitted. Thus, the Dirac Lagrangian becomes simply

$$
\mathcal{L}_{\text {Dirac }}=i \xi^{\dagger} \bar{\sigma}^{\mu} \partial_{\mu} \xi+i \chi \sigma^{\mu} \partial_{\mu} \chi^{\dagger}-m\left(\xi^{\dagger} \chi^{\dagger}+\chi \xi\right) .
$$


More generally, in the index-free notation:

$$
\begin{aligned}
\xi \chi & \equiv \xi^{\alpha} \chi_{\alpha}, & \xi^{\dagger} \chi^{\dagger} \equiv \xi_{\dot{\alpha}}^{\dagger} \chi^{\dagger \dot{\alpha}}, \\
\xi^{\dagger} \bar{\sigma}^{\mu} \chi & \equiv \xi_{\dot{\alpha}}^{\dagger} \bar{\sigma}^{\mu \dot{\alpha} \beta} \chi_{\beta}, & \xi \sigma^{\mu} \chi^{\dagger} \equiv \xi^{\alpha} \sigma_{\alpha \dot{\beta}}^{\mu} \chi^{\dagger \dot{\beta}} .
\end{aligned}
$$

Note that it is useful to regard spinors like $\psi^{\alpha}$ and $\psi_{\dot{\alpha}}^{\dagger}$ as row vectors, and $\psi^{\dagger \dot{\alpha}}$ and $\psi_{\alpha}$ as column vectors. As an exercise, you can now show that, with $\xi$ and $\chi$ anticommuting spinors,

$$
\begin{array}{ll}
\xi \chi=\chi \xi, & \xi^{\dagger} \chi^{\dagger}=\chi^{\dagger} \xi^{\dagger} \\
\xi^{\dagger} \bar{\sigma}^{\mu} \chi=-\chi \sigma^{\mu} \xi^{\dagger} . &
\end{array}
$$

For example, the Dirac Lagrangian can be rewritten yet again as:

$$
\mathcal{L}_{\text {Dirac }}=i \xi^{\dagger} \bar{\sigma}^{\mu} \partial_{\mu} \xi+i \chi^{\dagger} \bar{\sigma}^{\mu} \partial_{\mu} \chi-m\left(\xi \chi+\xi^{\dagger} \chi^{\dagger}\right),
$$

after discarding a total derivative. The Majorana Lagrangian is similarly:

$$
\mathcal{L}_{\text {Majorana }}=i \psi^{\dagger} \bar{\sigma}^{\mu} \partial_{\mu} \psi-\frac{1}{2} m\left(\psi \psi+\psi^{\dagger} \psi^{\dagger}\right) \text {. }
$$

Now, any theory of spin-1/2 fermions can be written in the 2-component fermion notation, with kinetic terms:

$$
\mathcal{L}=i \psi^{\dagger i} \bar{\sigma}^{\mu} \partial_{\mu} \psi_{i}-\frac{1}{2}\left(M^{i j} \psi_{i} \psi_{j}+\text { c.c. }\right)
$$

where $i$ is a flavor and/or gauge label and $M^{i j}$ is a mass matrix, and "c.c." denotes complex conjugation for classical fields. In general, it can be shown that a unitary rotation on the indices $i$ will put the mass matrix into a form where the only non-zero entries are diagonal entries $\mu_{i}$ and $2 \times 2$ blocks $\left(\begin{array}{cc}0 & m_{j} \\ m_{j} & 0\end{array}\right)$, with $\mu_{i}$ and $m_{j}$ all real and non-negative:

$$
\begin{aligned}
\mathcal{L}= & i \psi^{\dagger i} \bar{\sigma}^{\mu} \partial_{\mu} \psi_{i}-\frac{1}{2} \mu_{i}\left(\psi_{i} \psi_{i}+\psi^{\dagger i} \psi^{\dagger i}\right) \\
& +i \xi^{\dagger j} \bar{\sigma}^{\mu} \partial_{\mu} \xi_{j}+i \chi_{j}^{\dagger} \bar{\sigma}^{\mu} \partial_{\mu} \chi^{j}-m_{j}\left(\xi_{j} \chi^{j}+\xi^{\dagger j} \chi_{j}^{\dagger}\right),
\end{aligned}
$$

The resulting theory consists of Majorana fermions $\psi_{i}$ (for which diagonal mass terms are allowed by the symmetries), and Dirac fermions consisting of the pairs $\left(\xi_{j}, \chi^{j}\right)$.

The behavior of the spinor products under hermitian conjugation (for quantum field operators) or complex conjugation (for classical fields) is:

$$
\begin{aligned}
& (\xi \chi)^{\dagger}=\chi^{\dagger} \xi^{\dagger}, \\
& \left(\xi \sigma^{\mu} \chi^{\dagger}\right)^{\dagger}=\chi \sigma^{\mu} \xi^{\dagger}, \\
& \left(\xi^{\dagger} \bar{\sigma}^{\mu} \chi\right)^{\dagger}=\chi^{\dagger} \bar{\sigma}^{\mu} \xi \\
& \left(\xi \sigma^{\mu} \bar{\sigma}^{\nu} \chi\right)^{\dagger}=\chi^{\dagger} \bar{\sigma}^{\nu} \sigma^{\mu} \xi^{\dagger} .
\end{aligned}
$$

More generally,

$$
(\xi \Sigma \chi)^{\dagger}=\chi^{\dagger} \Sigma_{r} \xi^{\dagger}, \quad\left(\xi \Sigma \chi^{\dagger}\right)^{\dagger}=\chi \Sigma_{r} \xi^{\dagger}
$$


where in each case $\Sigma$ stands for any sequence of alternating $\sigma$ and $\bar{\sigma}$ matrices, and $\Sigma_{r}$ is obtained from $\Sigma$ by reversing the order of all of the $\sigma$ and $\bar{\sigma}$ matrices, since the sigma matrices are hermitian. Eqs. (2.1.30)-(2.1.34) are applicable both to anticommuting and to commuting spinors.

The following identities can be used to systematically simplify expressions involving products of $\sigma$ and $\bar{\sigma}$ matrices:

$$
\begin{aligned}
& \sigma_{\alpha \dot{\alpha}}^{\mu} \bar{\sigma}_{\mu}^{\dot{\beta} \beta}=-2 \delta_{\alpha}{ }^{\beta} \delta_{\dot{\alpha}}^{\dot{\beta}}, \\
& \sigma_{\alpha \dot{\alpha}}^{\mu} \sigma_{\mu \beta \dot{\beta}}=-2 \epsilon_{\alpha \beta} \epsilon_{\dot{\alpha} \dot{\beta}}, \\
& \bar{\sigma}^{\mu \dot{\alpha} \alpha} \bar{\sigma}_{\mu}^{\dot{\beta} \beta}=-2 \epsilon^{\alpha \beta} \epsilon^{\dot{\alpha} \dot{\beta}}, \\
& \left(\sigma^{\mu} \bar{\sigma}^{\nu}+\sigma^{\nu} \bar{\sigma}^{\mu}\right)_{\alpha}{ }^{\beta}=-2 g^{\mu \nu} \delta_{\alpha}{ }^{\beta}, \\
& \left(\bar{\sigma}^{\mu} \sigma^{\nu}+\bar{\sigma}^{\nu} \sigma^{\mu}\right)_{\dot{\beta}}^{\dot{\alpha}}=-2 g^{\mu \nu} \delta_{\dot{\beta}}^{\dot{\alpha}}, \\
& \sigma^{\mu} \bar{\sigma}^{\nu} \sigma^{\rho}=-g^{\mu \nu} \sigma^{\rho}+g^{\mu \rho} \sigma^{\nu}-g^{\nu \rho} \sigma^{\mu}-i \epsilon^{\mu \nu \rho \kappa} \sigma_{\kappa}, \\
& \bar{\sigma}^{\mu} \sigma^{\nu} \bar{\sigma}^{\rho}=-g^{\mu \nu} \bar{\sigma}^{\rho}+g^{\mu \rho} \bar{\sigma}^{\nu}-g^{\nu \rho} \bar{\sigma}^{\mu}+i \epsilon^{\mu \nu \rho \kappa} \bar{\sigma}_{\kappa} .
\end{aligned}
$$

Computations of cross-sections and decay rates generally require traces of alternating products of $\sigma$ and $\bar{\sigma}$ matrices:

$$
\begin{aligned}
& \operatorname{Tr}[1]=2, \\
& \operatorname{Tr}\left[\sigma^{\mu} \bar{\sigma}^{\nu}\right]=\operatorname{Tr}\left[\bar{\sigma}^{\mu} \sigma^{\nu}\right]=-2 g^{\mu \nu}, \\
& \operatorname{Tr}\left[\sigma^{\mu} \bar{\sigma}^{\nu} \sigma^{\rho} \bar{\sigma}^{\kappa}\right]=2\left(g^{\mu \nu} g^{\rho \kappa}-g^{\mu \rho} g^{\nu \kappa}+g^{\mu \kappa} g^{\nu \rho}+i \epsilon^{\mu \nu \rho \kappa}\right), \\
& \operatorname{Tr}\left[\bar{\sigma}^{\mu} \sigma^{\nu} \bar{\sigma}^{\rho} \sigma^{\kappa}\right]=2\left(g^{\mu \nu} g^{\rho \kappa}-g^{\mu \rho} g^{\nu \kappa}+g^{\mu \kappa} g^{\nu \rho}-i \epsilon^{\mu \nu \rho \kappa}\right) .
\end{aligned}
$$

Traces involving a larger even number of $\sigma$ and $\bar{\sigma}$ matrices can be systematically obtained from eqs. (2.1.42)-(2.1.45) by repeated use of eqs. (2.1.38) and (2.1.39) [and, if you are lucky, eqs. (2.1.35)-(2.1.37)], and the cyclic property of the trace. Traces involving an odd number of $\sigma$ and $\bar{\sigma}$ matrices cannot arise, because there is no way to connect the spinor indices consistently.

In addition to manipulating expressions containing anticommuting fermion quantum fields, one often must deal with products of commuting spinors that arise as external state wave functions in the Feynman rules. In the following expressions, a generic commuting or anticommuting spinor is denoted by by $z_{i}$, with the notation:

$$
(-1)^{A} \equiv\left\{\begin{array}{l}
+1, \text { commuting spinors } \\
-1, \text { anticommuting spinors. }
\end{array}\right.
$$

The following identities hold for the $z_{i}$ :

$$
\begin{aligned}
& z_{1} z_{2}=-(-1)^{A} z_{2} z_{1}, \\
& z_{1}^{\dagger} z_{2}^{\dagger}=-(-1)^{A} z_{2}^{\dagger} z_{1}^{\dagger}, \\
& z_{1} \sigma^{\mu} z_{2}^{\dagger}=(-1)^{A} z_{2}^{\dagger} \bar{\sigma}^{\mu} z_{1}, \\
& z_{1} \sigma^{\mu} \bar{\sigma}^{\nu} z_{2}=-(-1)^{A} z_{2} \sigma^{\nu} \bar{\sigma}^{\mu} z_{1}, \\
& z_{1}^{\dagger} \bar{\sigma}^{\mu} \sigma^{\nu} z_{2}^{\dagger}=-(-1)^{A} z_{2}^{\dagger} \bar{\sigma}^{\nu} \sigma^{\mu} z_{1}^{\dagger}, \\
& z_{1}^{\dagger} \bar{\sigma}^{\mu} \sigma^{\rho} \bar{\sigma}^{\nu} z_{2}=(-1)^{A} z_{2} \sigma^{\nu} \bar{\sigma}^{\rho} \sigma^{\mu} z_{1}^{\dagger} .
\end{aligned}
$$


The hermiticity properties of the spinor products in eqs. 2.1.30)-2.1.34) hold for both commuting and anticommuting spinors.

Two-component spinor products can often be simplified by using Fierz identities. Using the antisymmetry of the suppressed two-index epsilon symbol, you can show:

$$
\begin{aligned}
& \left(z_{1} z_{2}\right)\left(z_{3} z_{4}\right)=-\left(z_{1} z_{3}\right)\left(z_{4} z_{2}\right)-\left(z_{1} z_{4}\right)\left(z_{2} z_{3}\right) \\
& \left(z_{1}^{\dagger} z_{2}^{\dagger}\right)\left(z_{3}^{\dagger} z_{4}^{\dagger}\right)=-\left(z_{1}^{\dagger} z_{3}^{\dagger}\right)\left(z_{4}^{\dagger} z_{2}^{\dagger}\right)-\left(z_{1}^{\dagger} z_{4}^{\dagger}\right)\left(z_{2}^{\dagger} z_{3}^{\dagger}\right)
\end{aligned}
$$

where eqs. 2.1.47) and (2.1.48) have been used to eliminate any residual factors of $(-1)^{A}$. Additional Fierz identities follow from eqs. (2.1.35)-(2.1.37):

$$
\begin{aligned}
& \left(z_{1} \sigma^{\mu} z_{2}^{\dagger}\right)\left(z_{3}^{\dagger} \bar{\sigma}_{\mu} z_{4}\right)=2\left(z_{1} z_{4}\right)\left(z_{2}^{\dagger} z_{3}^{\dagger}\right), \\
& \left(z_{1}^{\dagger} \bar{\sigma}^{\mu} z_{2}\right)\left(z_{3}^{\dagger} \bar{\sigma}_{\mu} z_{4}\right)=-2\left(z_{1}^{\dagger} z_{3}^{\dagger}\right)\left(z_{4} z_{2}\right), \\
& \left(z_{1} \sigma^{\mu} z_{2}^{\dagger}\right)\left(z_{3} \sigma_{\mu} z_{4}^{\dagger}\right)=-2\left(z_{1} z_{3}\right)\left(z_{4}^{\dagger} z_{2}^{\dagger}\right) .
\end{aligned}
$$

Eqs. (2.1.53)-(2.1.57) hold for both commuting and anticommuting spinors.

The preceding identities hold in the case that the number of spacetime dimensions is exactly 4 . This is appropriate for tree-level computations, but in calculations of radiative corrections one often makes use of regularization by dimensional continuation to $d$ dimensions, where $d$ is infinitesimally different from 4 . For non-supersymmetric theories, the most common method is the classic dimensional regularization method [13, while in supersymmetry one uses some version of dimensional reduction [14] in order to avoid spurious violations of supersymmetry due to a mismatch between the gaugino and gauge boson degrees of freedom.

When using dimensional continuation regulators, some identities that would hold in unregularized four-dimensional theories are simply inconsistent and must not be used; other identities remain valid if $d$ replaces 4 in the appropriate spots; and still other identities hold without modification. Some important identities that do hold in $d \neq 4$ dimensions are eqs. (2.1.38) and (2.1.39), and the trace identities eqs. (2.1.42) and (2.1.43).

In contrast, the Fierz identities eqs. (2.1.35), (2.1.36), and (2.1.37), and eqs. (2.1.55), (2.1.56), and (2.1.57), do not have a consistent, unambiguous meaning for $d \neq 4$. However, the following identities that are implied by these equations in $d=4$ do consistently generalize to $d \neq 4$ :

$$
\begin{aligned}
& {\left[\sigma^{\mu} \bar{\sigma}_{\mu}\right]_{\alpha}^{\beta}=-d \delta_{\alpha}^{\beta},} \\
& {\left[\bar{\sigma}^{\mu} \sigma_{\mu}\right]_{\dot{\beta}}^{\dot{\alpha}}=-d \delta_{\dot{\beta}}^{\dot{\alpha}} .}
\end{aligned}
$$

Taking these and repeatedly using eqs. (2.1.38) and (2.1.39) then yields:

$$
\begin{aligned}
& {\left[\sigma^{\mu} \bar{\sigma}^{\nu} \sigma_{\mu}\right]_{\alpha \dot{\beta}}=(d-2) \sigma_{\alpha \dot{\beta}}^{\nu},} \\
& {\left[\bar{\sigma}^{\mu} \sigma_{\nu} \bar{\sigma}_{\mu}\right]^{\dot{\alpha} \beta}=(d-2) \bar{\sigma}_{\nu}^{\dot{\alpha} \beta},} \\
& {\left[\sigma^{\mu} \bar{\sigma}^{\nu} \sigma^{\rho} \bar{\sigma}_{\mu}\right]_{\alpha}^{\beta}=4 g^{\nu \rho} \delta_{\alpha}^{\beta}+(4-d)\left[\sigma^{\nu} \bar{\sigma}^{\rho}\right]_{\alpha}{ }^{\beta},} \\
& {\left[\bar{\sigma}^{\mu} \sigma^{\nu} \bar{\sigma}^{\rho} \sigma_{\mu}\right]_{\dot{\beta}}^{\dot{\alpha}}=4 g^{\nu \rho} \delta_{\dot{\beta}}^{\dot{\alpha}}+(4-d)\left[\bar{\sigma}^{\nu} \sigma^{\rho}\right]_{\dot{\beta}}^{\dot{\alpha}},}
\end{aligned}
$$




$$
\begin{aligned}
& {\left[\sigma^{\mu} \bar{\sigma}^{\nu} \sigma^{\rho} \bar{\sigma}^{\kappa} \sigma_{\mu}\right]_{\alpha \dot{\beta}}=2\left[\sigma^{\kappa} \bar{\sigma}^{\rho} \sigma^{\nu}\right]_{\alpha \dot{\beta}}-(4-d)\left[\sigma^{\nu} \bar{\sigma}^{\rho} \sigma^{\kappa}\right]_{\alpha \dot{\beta}},} \\
& {\left[\bar{\sigma}^{\mu} \sigma^{\nu} \bar{\sigma}^{\rho} \sigma^{\kappa} \bar{\sigma}_{\mu}\right]^{\dot{\alpha} \beta}=2\left[\bar{\sigma}^{\kappa} \sigma^{\rho} \bar{\sigma}^{\nu}\right]^{\dot{\alpha} \beta}-(4-d)\left[\bar{\sigma}^{\nu} \sigma^{\rho} \bar{\sigma}^{\kappa}\right]^{\dot{\alpha} \beta} .}
\end{aligned}
$$

Identities that involve the (explicitly and inextricably four-dimensional) $\epsilon^{\mu \nu \rho \kappa}$ symbol, such as eqs. (2.1.40), (2.1.41), (2.1.44), and (2.1.45), are also only meaningful in exactly four dimensions. This can lead to ambiguities or inconsistencies in loop computations where it is necessary to perform the computation in $d \neq 4$ dimensions; see, for example, refs. [15]-[19]. Fortunately, in practice one typically finds that the above expressions appear multiplied by the metric and/or other external tensors, such as linearly dependent four-momenta appropriate to the problem at hand. In almost all such cases, two of the indices appearing in the traces are symmetrized, which eliminates the $\epsilon^{\mu \nu \rho \kappa}$ term, rendering the resulting expressions unambiguous. For example, one can write

$$
\operatorname{Tr}\left[\sigma^{\mu} \bar{\sigma}^{\nu} \sigma^{\rho} \bar{\sigma}^{\kappa}\right]+\operatorname{Tr}\left[\bar{\sigma}^{\mu} \sigma^{\nu} \bar{\sigma}^{\rho} \sigma^{\kappa}\right]=4\left(g^{\mu \nu} g^{\rho \kappa}-g^{\mu \rho} g^{\nu \kappa}+g^{\mu \kappa} g^{\nu \rho}\right) .
$$

unambiguously in $d \neq 4$ dimensions. In other cases, one can separate a calculation into a divergent part without ambiguities plus a convergent part which would be ambiguous in $d \neq 4$, but which can be safely evaluated in $d=4$. Sometimes this requires first combining the contributions of several Feynman diagrams. This is the case in the triangle anomaly calculation using 2-component fermions, discussed in depth in ref. [12].

\subsection{Fermion interaction vertices}

In renormalizable quantum field theories, fermions can interact either with scalars or with vector fields. In 2-component language, the scalar-fermion-fermion interactions can be written as:

$$
\mathcal{L}_{\text {int }}=-\frac{1}{2} Y^{I j k} \phi_{I} \psi_{j} \psi_{k}-\frac{1}{2} Y_{I j k} \phi^{I} \psi^{\dagger j} \psi^{\dagger k}
$$

where the fields are assumed to be mass eigenstates, and $Y^{I j k}$ are dimensionless Yukawa couplings with $Y_{I j k}=\left(Y^{I j k}\right)^{*}$. The 2-component fields $\psi_{i}$ make be either Majorana or parts of Dirac fermions, and the spinor indices have been suppressed. The scalar fields $\phi_{I}$ may be either real or complex, with $\phi^{I} \equiv\left(\phi_{I}\right)^{*}$. The corresponding Feynman rules are obtained as usual by multiplying the couplings in the Lagrangian by $i$, and are shown in Figure 2.2.1.

In contrast to 4-component Feynman rules, the directions of arrows in 2-component Feynman rules do not correspond to the flow of charge or fermion number. Instead, the arrows indicate the spinor index structure, with fields of undotted indices flowing into any vertex and fields of dotted indices flowing out of any vertex. This corresponds to the fact that the 2-component fields are distinguished by their Lorentz group transformation properties, rather than their status as particle or antiparticle as in 4-component notation. For the interactions of scalars in Figure 2.2.1, the spinor indices are always just proportional to the identity matrix, and so can be trivially suppressed. For this reason, we will always just omit the spinor indices on scalar-fermion-fermion interaction Feynman rules in later sections. 
(a)

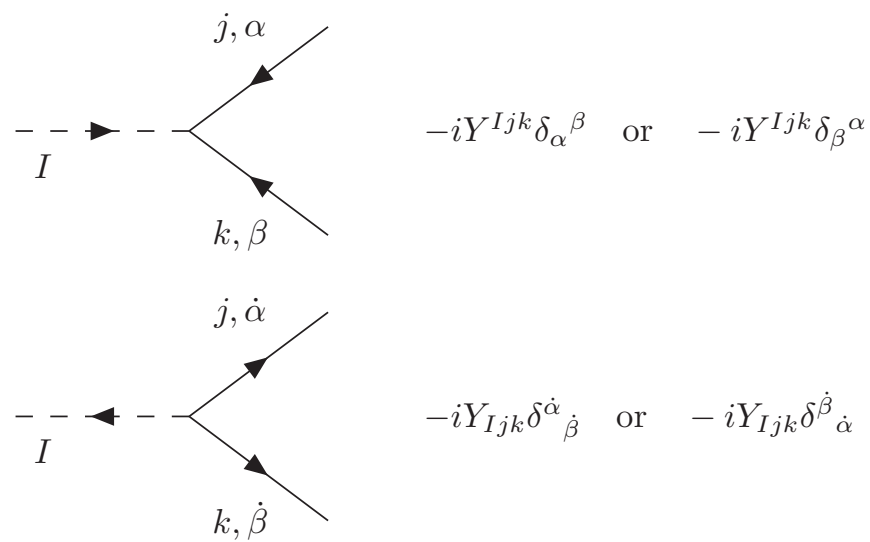

Fig. 2.2.1: Feynman rules for Yukawa couplings of scalars to 2-component fermions in a general field theory. The choice of which rule to use depends on how the vertex connects to the rest of the amplitude. When spinor indices are suppressed, the Kronecker $\delta$ 's are trivial in either case, so we will not show the explicit spinor indices in specific realizations of this rule below.

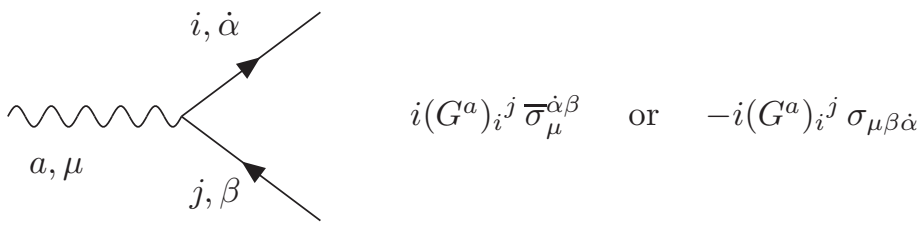

Fig. 2.2.2: The Feynman rules for 2-component fermion interactions with gauge bosons. Which one should be used depends on how the vertex connects to the rest of the diagram. The $G^{a}$ are defined in eq. (2.2.2). In specific realizations of this rule below, we will not explicitly show the spinor indices; it is understood that the dotted index is associated with an outgoing line and the undotted with the incoming line. Also, we will only show the $\bar{\sigma}_{\mu}$ version of the rule; there is always another version of the rule with $\bar{\sigma}_{\mu} \rightarrow-\sigma_{\mu}$.

Next consider fermion interactions with vector fields. The general form of the interactions of 2-component fermions with vector bosons $A_{a}^{\mu}$ labeled by an index $a$ is:

$$
\mathcal{L}_{\text {int }}=\left(G^{a}\right)_{i}{ }^{j} A_{a}^{\mu} \psi^{\dagger i} \bar{\sigma}_{\mu} \psi_{j} .
$$

Here $G^{a}$ is a dimensionless coupling matrix, which in the special case that the fields are gauge eigenstates is given by $g_{a} \boldsymbol{T}^{a}$, where $g_{a}$ and $\boldsymbol{T}^{a}$ are the gauge coupling and fermion representation matrix of the theory. In general, the form of eq. (2.2.2) is the result of diagonalizing both the vector and fermion mass matrices. The corresponding 2-component fermion Feynman rules are shown in Figure 2.2.2. Note that there are two different forms for the Feynman rule, one proportional to $\bar{\sigma}_{\mu}$ and the other to $-\sigma_{\mu}$. Which one should be used depends on how the vertex is connect to the rest of the amplitude; the spinor indices will connect in the only way possible. Below, when presenting Feynman rules for specific vector-fermion-fermion interactions, we will always have an outgoing arrow at the top (corresponding implicitly to a dotted index $\dot{\alpha}$ ) and an incoming arrow at the bottom (for an undotted index $\beta$ ), and simply write $\bar{\sigma}_{\mu}$ rather 


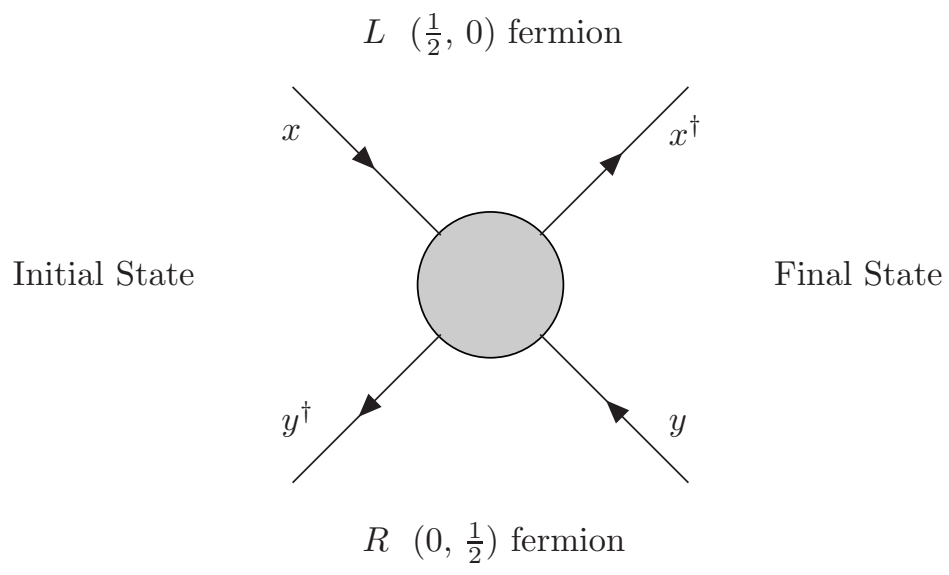

Fig. 2.3.1: Mnemonic for the assignment of external wave function spinors, for initial and final state left-handed $\left(\frac{1}{2}, 0\right)$ and right-handed $\left(0, \frac{1}{2}\right)$ fermions.

than $\bar{\sigma}_{\mu}^{\dot{\alpha} \beta}$, and with the understanding that there is always a corresponding rule with $\bar{\sigma}_{\mu} \rightarrow-\sigma_{\mu}$. Thus the structure of each such Feynman rule will be exactly like Figure 2.2.2, but with the indices suppressed for simplicity of presentation.

\subsection{External wavefunctions for 2-component spinors}

In the standard textbook calculations in 4-component spinor language, one makes use of external wavefunction spinors $u, \bar{v}, \bar{u}, v$, for, respectively, initial state fermions, initial state anti-fermions, final state fermions, and final state anti-fermions. Similarly, when doing calculation in the 2-component formalism, one makes use of external wavefunction 2-component spinors:

$$
x_{\alpha}(\vec{p}, s), \quad y_{\dot{\alpha}}^{\dagger}(\vec{p}, s), \quad x_{\dot{\alpha}}^{\dagger}(\vec{p}, s), \quad y_{\alpha}(\vec{p}, s),
$$

for, respectively, initial-state left-handed $\left(\frac{1}{2}, 0\right)$, initial-state right-handed $\left(0, \frac{1}{2}\right)$, finalstate left-handed $\left(\frac{1}{2}, 0\right)$, and final-state right-handed $\left(0, \frac{1}{2}\right)$ states. See Figure 2.3.1 for a mnemonic. These external wave function spinors are commuting (Grassmann-even) objects, despite carrying spinor indices, and are applicable to both Dirac and Majorana fermions. They depend on the three-momentum $\vec{p}$ and the spin $s$ of particle, and are related to the usual representation of the 4-component $u$ and $v$ spinors by

$$
u(\vec{p}, s)=\left(\begin{array}{c}
x_{\alpha}(\vec{p}, s) \\
y^{\dagger \dot{\alpha}}(\vec{p}, s)
\end{array}\right), \quad v(\vec{p}, s)=\left(\begin{array}{c}
y_{\alpha}(\vec{p}, s) \\
x^{\dagger \dot{\alpha}}(\vec{p}, s)
\end{array}\right) .
$$

In the following, we will only consider problems for which the spin states $s$ are summed over. In that case, the explicit forms of the spinors $x, y, x^{\dagger}, y^{\dagger}$ are not needed a Instead,

$\overline{{ }^{a} \text { See ref. [12] for the explicit forms of } x, y, x^{\dagger}, y^{\dagger}}$, and examples with spins not summed. 
one makes use of the spin-sum identities

$$
\begin{array}{cl}
\sum_{s} x_{\alpha}(\overrightarrow{\boldsymbol{p}}, s) x_{\dot{\beta}}^{\dagger}(\overrightarrow{\boldsymbol{p}}, s)=-p \cdot \sigma_{\alpha \dot{\beta}}, & \sum_{s} x^{\dagger \dot{\alpha}}(\overrightarrow{\boldsymbol{p}}, s) x^{\beta}(\overrightarrow{\boldsymbol{p}}, s)=-p \cdot \bar{\sigma}^{\dot{\alpha} \beta}, \\
\sum_{s} y^{\dagger \dot{\alpha}}(\overrightarrow{\boldsymbol{p}}, s) y^{\beta}(\overrightarrow{\boldsymbol{p}}, s)=-p \cdot \bar{\sigma}^{\dot{\alpha} \beta}, & \sum_{s} y_{\alpha}(\overrightarrow{\boldsymbol{p}}, s) y_{\dot{\beta}}^{\dagger}(\overrightarrow{\boldsymbol{p}}, s)=-p \cdot \sigma_{\alpha \dot{\beta}}, \\
\sum_{s} x_{\alpha}(\overrightarrow{\boldsymbol{p}}, s) y^{\beta}(\overrightarrow{\boldsymbol{p}}, s)=m \delta_{\alpha}^{\beta}, & \sum_{s} y_{\alpha}(\overrightarrow{\boldsymbol{p}}, s) x^{\beta}(\overrightarrow{\boldsymbol{p}}, s)=-m \delta_{\alpha}{ }^{\beta}, \\
\sum_{s} y^{\dagger \dot{\alpha}}(\overrightarrow{\boldsymbol{p}}, s) x_{\dot{\beta}}^{\dagger}(\overrightarrow{\boldsymbol{p}}, s)=m \delta_{\dot{\beta}}^{\dot{\alpha}}, & \sum_{s} x^{\dagger \dot{\alpha}}(\overrightarrow{\boldsymbol{p}}, s) y_{\dot{\beta}}^{\dagger}(\overrightarrow{\boldsymbol{p}}, s)=-m \delta_{\dot{\beta}}^{\dot{\alpha}},
\end{array}
$$

where $m$ and $p^{\mu}$ are the mass and 4-momentum of the fermion. They also obey useful reduction identities:

$$
\begin{aligned}
(p \cdot \bar{\sigma})^{\dot{\alpha} \beta} x_{\beta}=-m y^{\dagger \dot{\alpha}}, & (p \cdot \sigma)_{\alpha \dot{\beta}} y^{\dagger \dot{\beta}}=-m x_{\alpha}, \\
(p \cdot \sigma)_{\alpha \dot{\beta}} x^{\dagger \dot{\beta}}=m y_{\alpha}, & (p \cdot \bar{\sigma})^{\dot{\alpha} \beta} y_{\beta}=m x^{\dagger \dot{\alpha}}, \\
x^{\alpha}(p \cdot \sigma)_{\alpha \dot{\beta}}=m y_{\dot{\beta}}^{\dagger}, & y_{\dot{\alpha}}^{\dagger}(p \cdot \bar{\sigma})^{\dot{\alpha} \beta}=m x^{\beta}, \\
x_{\dot{\alpha}}^{\dagger}(p \cdot \bar{\sigma})^{\dot{\alpha} \beta}=-m y^{\beta}, & y^{\alpha}(p \cdot \sigma)_{\alpha \dot{\beta}}=-m x_{\dot{\beta}}^{\dagger},
\end{aligned}
$$

which are on-shell conditions embodying the classical equations of motion of the freefield Lagrangian.

\subsection{Propagators}

Fermion propagators for 2-component fermions are of two types. The first type preserves the arrow direction on the fermion line, and therefore carries one dotted and one undotted index. The second type does not preserve the arrow direction, and therefore has either two dotted or two undotted indices.

The Feynman rule for the arrow-preserving propagator for any fermion of mass $m$ is shown in Figure 2.4.1. (For simplicity of notation, the $-i \epsilon$ terms in the denominators are omitted in all propagator rules.) Note that for the arrow-preserving propagator of Figure 2.4.2(a), there are two versions, depending on how the spinor indices are connected to the rest of the amplitude. The 4-momentum $p^{\mu}$ is taken to flow in the direction indicated.

The propagators with arrows clashing correspond to an odd number of mass insertions. The corresponding Feynman rules are shown in Figure 2.4.2 for a Majorana fermion, and in Figure 2.4.3 for a Dirac fermion of mass $m$ consisting of two 2-component fermions $\chi$ and $\xi$, as in eq. (2.1.26). Note that while the arrow-preserving propagators

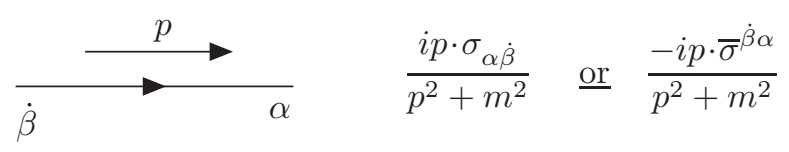

Fig. 2.4.1: Two-component Feynman rule for arrow-preserving propagator of a Majorana or Dirac fermion with mass $m$. 
(a)

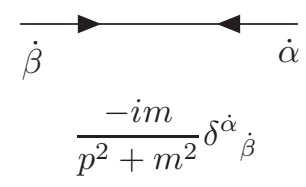

(b)

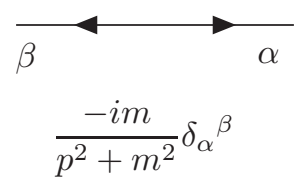

Fig. 2.4.2: Two-component Feynman rules for arrow-clashing propagator of a Majorana fermion with mass $m$.

(a)

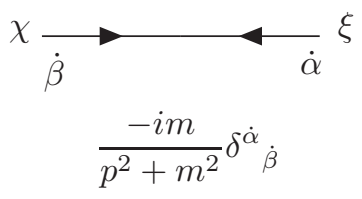

(b)

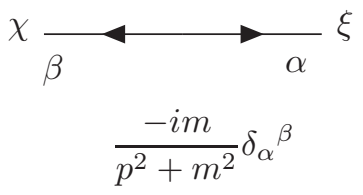

Fig. 2.4.3: Feynman rules for arrow-clashing propagators of a pair of charged 2-component fermions $\chi, \xi$ with a Dirac mass $m$.

$$
\begin{array}{ll}
------ & \frac{-i}{p^{2}+m^{2}} \\
\sim \sim \sim & \frac{-i}{p^{2}+m^{2}}\left[g^{\mu \nu}-(1-\xi) \frac{p^{\mu} p^{\nu}}{p^{2}+\xi m^{2}}\right]
\end{array}
$$

Fig. 2.4.4: Feynman rules for the (neutral or charged) scalar and gauge boson propagators, in the $R_{\xi}$ gauge, where $p^{\mu}$ is the propagating four-momentum. In the gauge boson propagator, $\xi=1$ defines the 't Hooft-Feynman gauge, $\xi=0$ defines the Landau gauge, and $\xi \rightarrow \infty$ defines the unitary gauge.

never change the identity of the 2-component fermion, in the case of Dirac fermions the propagators with clashing arrows always connect the two oppositely charged members of the Dirac pair $(\chi$ and $\xi)$.

For completeness, Figure 2.4.4 shows the Feynman rules for bosons in the same conventions.

\subsection{General structure and rules for Feynman graphs}

When computing an amplitude for a given process, all possible diagrams should be drawn that conform with the rules given above for external wave functions, interactions, and propagators. For each contributing diagram, one writes down the amplitude as follows. Starting from any external wave function spinor (or from any vertex on a fermion loop), factors corresponding to each propagator and vertex should be written down from left to right, following the fermion line until it ends at another external state wave function (or at the original point on the fermion loop). If one starts a fermion line at an $x$ or $y$ external state spinor, it should have a raised undotted index in accord with eq. (2.1.20). Or, if one starts with an $x^{\dagger}$ or $y^{\dagger}$, it should have a lowered dotted spinor index. Then, all spinor indices should always be contracted as in eq. (2.1.20). If one ends with an $x$ or $y$ external state spinor, it will have a lowered undotted index, while if one ends with an $x^{\dagger}$ or $y^{\dagger}$ spinor, it will have a raised dotted index. For arrow-preserving fermion propagators, and for gauge vertices, the preceding determines whether the $\sigma$ 
or $\bar{\sigma}$ rule should be used. For closed fermion loops, one must choose a direction around the loop for writing down contributions; then the $\bar{\sigma}(\sigma)$ version of the arrow-preserving propagator rule should be used when the arrow is being followed forwards (backwards).

With these rules, spinor indices will be naturally suppressed so that:

- No explicit 2-component $\epsilon$ symbols appear.

- For any amplitude, factors of $\sigma$ and $\bar{\sigma}$ must alternate.

- An $x$ and $y$ may be followed by a $\sigma$ or preceded by a $\bar{\sigma}$, but not followed by a $\bar{\sigma}$ or preceded by a $\sigma$. Similarly, an $x^{\dagger}$ and $y^{\dagger}$ may be followed by a $\bar{\sigma}$ or preceded by a $\sigma$, but may not be followed by a $\sigma$ or preceded by a $\bar{\sigma}$.

For any given process, different contributing diagrams may (and usually will) have different external state wave function spinors for the same external fermion.

Symmetry factors for identical particles are implemented in the usual way. FermiDirac statistics are implemented by the following rules:

- Each closed fermion loop gets a factor of -1 .

- A relative minus sign is imposed between terms contributing to a given amplitude whenever the ordering of external state spinors (written left-to-right in a formula) differs by an odd permutation.

Notice that there is freedom to choose which direction each fermion line in a diagram is traversed while applying the above rules. However, for each diagram one must include a sign that depends on the ordering of the external fermions. This sign can be fixed by first choosing some canonical ordering of the external fermions. Then for any diagram that contributes to the process of interest, the corresponding sign is positive (negative) if the ordering of external fermions is an even (odd) permutation with respect to the canonical ordering. If one chooses a different canonical ordering, then the total resulting amplitude may change by an overall sign. This is consistent with the fact that the $S$ matrix element is only defined up to an overall phase, which is not physically observable.

Amplitudes generated according to these rules will contain objects:

$$
a=z_{1} \Sigma z_{2}
$$

where $z_{1}$ and $z_{2}$ are each commuting external spinor wave functions $x, x^{\dagger}, y$, or $y^{\dagger}$, and $\Sigma$ is a sequence of alternating $\sigma$ and $\bar{\sigma}$ matrices. The complex conjugate of $a$ is obtained by applying the results of eqs. (2.1.30)-(2.1.34):

$$
a^{*}=z_{2}^{\dagger} \Sigma_{r} z_{1}^{\dagger}
$$

where $\Sigma_{r}$ is obtained from $\Sigma$ by reversing the order of all the $\sigma$ and $\bar{\sigma}$ matrices.

Section 5 provides some examples to illustrate the preceding rules.

\subsection{Conventions for names and fields of fermions and antifermions}

Let us now specify conventions for labeling Feynman diagrams that contain 2component fermion fields of the Standard Model (SM) and its minimal supersymmetric 
Table 2.6.1: Fermion and antifermion names and 2-component fields in the Standard Model and the MSSM. In the listing of 2-component fields, the first is an undaggered $\left(\frac{1}{2}, 0\right)$ [left-handed] field and the second is a daggered $\left(0, \frac{1}{2}\right)$ [right-handed] field. The bars on the 2-component (antifermion) fields are part of their names, and do not denote any form of complex conjugation. (In this table, neutrinos are considered to be exactly massless and there are no left-handed antineutrinos $\bar{\nu}$.)

\begin{tabular}{|c|c|c|}
\hline Fermion name & 2-component fields & Mass type \\
\hline \hline$\ell^{-}$(lepton) & $\ell, \bar{\ell}^{\dagger}$ & Dirac \\
\hline$\ell^{+}$(anti-lepton) & $\bar{\ell}, \ell^{\dagger}$ & Dirac \\
\hline$\nu$ (neutrino) & $\nu,-$ & Weyl \\
\hline $\bar{\nu}$ (antineutrino) &,$- \nu^{\dagger}$ & Weyl \\
\hline$q$ (quark) & $q, \bar{q}^{\dagger}$ & Dirac \\
\hline $\bar{q}$ (anti-quark) & $\bar{q}, q^{\dagger}$ & Dirac \\
\hline$\widetilde{N}_{i}$ (neutralino) & $\chi_{i}^{0}, \chi_{i}^{0^{\dagger}}$ & Majorana \\
\hline$\widetilde{C}_{i}^{+}$(chargino) & $\chi_{i}^{+}, \chi_{i}^{{ }^{\dagger}}$ & Dirac \\
\hline$\widetilde{C}_{i}^{-}$(anti-chargino) & $\chi_{i}^{-}, \chi_{i}^{+^{\dagger}}$ & Dirac \\
\hline$\widetilde{g}$ (gluino) & $\widetilde{g}, \widetilde{g}^{\dagger}$ & Majorana \\
\hline
\end{tabular}

extension (MSSM). In the case of Majorana fermions, things are easy because there is a one-to-one correspondence between the particle names and the undaggered $\left(\frac{1}{2}, 0\right)$ [lefthanded] fields. In contrast, for Dirac fermions there are always two distinct 2-component fields that correspond to each particle name. For a quark or lepton generically denoted by the particle name $f$, we call the 2 -component undaggered $\left(\frac{1}{2}, 0\right)$ [left-handed] fields $f$ and $\bar{f}$. This is illustrated in Table 2.6.1, which lists the SM and MSSM fermion particle names together with the corresponding 2-component fields. For each particle, we list the 2-component field(s) with the same quantum numbers. Because some of the symbols used as particle names also appear as names for the 2-component fields, one should make clear explicitly or from the context which is meant.

The neutralino and chargino cases deserve special attention. As particles, they are given the names $\widetilde{N}_{i}(i=1,2,3,4)$ and $\widetilde{C}_{i}^{ \pm}(i=1,2)$, respectively $\mathrm{b}$ As fields, however, there are two distinct 2-component chargino fields, which we call $\chi_{i}^{+}$and $\chi_{i}^{-}$; these are not conjugates of each other, just like the distinct 2-component fields $e$ and $\bar{e}$ for the electron. In the case of Majorana fields, one must also distinguish between the $\chi_{i}^{0}$ and $\chi_{i}^{0 \dagger}$ fields for the neutralino, and similarly for the gluino fields $\widetilde{g}$ and $\widetilde{g}^{\dagger}$. Here, the particle name is also $\widetilde{g}$.

\footnotetext{
${ }^{\mathrm{b}}$ It is also popular to call these particles by the names $\chi$ instead. However, the letter names $N, C$ are easier to visually recognize, and are better for efficient blackboard scribbling and informal electronic communications such as email, texting, and social media. So, everyone should switch to the convention of writing the particle names as $N, C$.
} 
There is now a choice to be made; should fermion lines in Feynman diagrams be labeled by particle names or by field names? Each choice has advantages and disadvantages. To eliminate the possibility of ambiguity, we always label fermion lines with 2-component fields (rather than particle names), and adopt the following conventions:

- In Feynman rules for interaction vertices, the external lines are always labeled by the undaggered $\left(\frac{1}{2}, 0\right)$ [left-handed] field, regardless of whether the corresponding arrow is pointed in or out of the vertex. Two-component fermion lines with arrows pointing away from the vertex correspond to dotted indices, and two-component fermion lines with arrows pointing toward the vertex always correspond to undotted indices.

- Internal fermion lines in Feynman diagrams are also always labeled by the undaggered field(s). Internal fermion lines containing a propagator with opposing arrows can carry two labels if the fermion is Dirac.

- Initial state external fermion lines in Feynman diagrams for complete processes are labeled by the corresponding undaggered $\left(\frac{1}{2}, 0\right)$ [left-handed] field if the arrow is into the vertex, and by the daggered $\left(0, \frac{1}{2}\right)$ [right-handed] field if the arrow is away from the vertex.

- Final state external fermion lines in Feynman diagrams for complete processes are labeled by the corresponding daggered $\left(0, \frac{1}{2}\right)$ [right-handed] field if the arrow is into the vertex, and by the undaggered $\left(\frac{1}{2}, 0\right)$ [left-handed] field if the arrow is away from the vertex.

\section{Feynman rules for fermions in the Standard Model}

Let us now review how the Standard Model quarks and leptons are described in this notation. The complete list of left-handed Weyl spinors in the Standard Model consists of $S U(2)_{L}$ doublets:

$$
Q_{i}=\left(\begin{array}{c}
u \\
d
\end{array}\right),\left(\begin{array}{c}
c \\
s
\end{array}\right),\left(\begin{array}{c}
t \\
b
\end{array}\right) ; \quad L_{i}=\left(\begin{array}{c}
\nu_{e} \\
e
\end{array}\right),\left(\begin{array}{c}
\nu_{\mu} \\
\mu
\end{array}\right),\left(\begin{array}{c}
\nu_{\tau} \\
\tau
\end{array}\right)
$$

and $S U(2)_{L}$ singlets:

$$
\bar{u}^{i}=\bar{u}, \bar{c}, \bar{t} ; \quad \bar{d}^{i}=\bar{d}, \bar{s}, \bar{b} ; \quad \bar{e}^{i}=\bar{e}, \bar{\mu}, \bar{\tau} .
$$

Here $i=1,2,3$ is a family index. The bars on the $S U(2)_{L}$-singlet fields are parts of their names, and do not denote any kind of conjugation. Rather, the unbarred fields are the left-handed pieces of a Dirac spinor, while the barred fields are the names given to the conjugates of the right-handed piece of a Dirac spinor. For example, the electron's 4-component Dirac field is $\left(\begin{array}{c}e_{\alpha} \\ \bar{e}^{\dagger \dot{\alpha}}\end{array}\right)$ and similarly for all of the other quark and charged lepton Dirac spinors. (The neutrinos of the Standard Model are not part of a Dirac spinor, at least in the approximation that they are massless.) The weak isodoublet fields $Q_{i}$ and $L_{i}$ always go together when one is constructing interactions invariant under 
the full Standard Model gauge group $S U(3)_{C} \times S U(2)_{L} \times U(1)_{Y}$. Suppressing all color and weak isospin indices, the kinetic and gauge part of the Standard Model fermion Lagrangian density is then

$$
\begin{aligned}
\mathcal{L}= & i Q^{\dagger i} \bar{\sigma}^{\mu} \nabla_{\mu} Q_{i}+i \bar{u}_{i}^{\dagger} \bar{\sigma}^{\mu} \nabla_{\mu} \bar{u}^{i}+i \bar{d}_{i}^{\dagger} \bar{\sigma}^{\mu} \nabla_{\mu} \bar{d}^{i} \\
& +i L^{\dagger i} \bar{\sigma}^{\mu} \nabla_{\mu} L_{i}+i \bar{e}_{i}^{\dagger} \bar{\sigma}^{\mu} \nabla_{\mu} \bar{e}^{i},
\end{aligned}
$$

with the family index $i$ summed over, and $\nabla_{\mu}$ the appropriate Standard Model covariant derivative. For example,

$$
\begin{aligned}
\nabla_{\mu}\left(\begin{array}{c}
\nu_{e} \\
e
\end{array}\right) & =\left[\partial_{\mu}-i g W_{\mu}^{a}\left(\tau^{a} / 2\right)-i g^{\prime} Y_{L} B_{\mu}\right]\left(\begin{array}{c}
\nu_{e} \\
e
\end{array}\right), \\
\nabla_{\mu} \bar{e} & =\left[\partial_{\mu}-i g^{\prime} Y_{\bar{e}} B_{\mu}\right] \bar{e},
\end{aligned}
$$

with $\tau^{a}(a=1,2,3)$ equal to the Pauli matrices, $Y_{L}=-1 / 2$ and $Y_{\bar{e}}=+1$. The gauge eigenstate weak bosons are related to the mass eigenstates by

$$
\begin{aligned}
W_{\mu}^{ \pm} & =\left(W_{\mu}^{1} \mp i W_{\mu}^{2}\right) / \sqrt{2}, \\
\left(\begin{array}{c}
Z_{\mu} \\
A_{\mu}
\end{array}\right) & =\left(\begin{array}{cc}
\cos \theta_{W} & -\sin \theta_{W} \\
\sin \theta_{W} & \cos \theta_{W}
\end{array}\right)\left(\begin{array}{c}
W_{\mu}^{3} \\
B_{\mu}
\end{array}\right) .
\end{aligned}
$$

Similar expressions hold for the other quark and lepton gauge eigenstates, with $Y_{Q}=1 / 6$, $Y_{\bar{u}}=-2 / 3$, and $Y_{\bar{d}}=1 / 3$. The quarks also have a term in the covariant derivative corresponding to gluon interactions proportional to $g_{3}$ (with $\alpha_{S}=g_{3}^{2} / 4 \pi$ ) with generators $\boldsymbol{T}^{a}=\lambda^{a} / 2$ for $Q$, and in the complex conjugate representation $\boldsymbol{T}^{a}=-\left(\lambda^{a}\right)^{*} / 2$ for $\bar{u}$ and $\bar{d}$, where $\lambda^{a}$ are the Gell-Mann matrices.

The corresponding Feynman rules for Standard Model fermion interactions with vector bosons are shown in Figures 3.1 and 3.2 for electroweak and QCD, respectively. The indices $i$ and $j$ label the fermion generations; an upper [lowered] flavor index in the corresponding Feynman rule is associated with a fermion line that points into [out from] the vertex. The couplings of the fermions to $\gamma$ and $Z$ and gluons are flavor-diagonal. For the $W^{ \pm}$bosons, the charge indicated is flowing into the vertex. The electric charge is denoted by $Q_{f}$ (in units of $e>0$ ), with $Q_{e}=-1$ for the electron. $T_{3}^{f}=1 / 2$ for $f=u$, $\nu$, and $T_{3}^{f}=-1 / 2$ for $f=d, \ell$. The Cabibbo-Kobayashi-Maskawa (CKM) mixing matrix is denoted by $\boldsymbol{K}$, with $\boldsymbol{K}_{1}{ }^{1}=V_{u d}$, and $\boldsymbol{K}_{2}{ }^{3}=V_{c b}$, etc. Also $s_{W} \equiv \sin \theta_{W}, c_{W} \equiv \cos \theta_{W}$ and $e \equiv g \sin \theta_{W}$.

In the Standard Model, each of the quark and lepton couplings to the Higgs boson $h$ has the form

$$
\mathcal{L}_{\text {Yukawa }}=-\frac{Y_{f}}{\sqrt{2}} h(f \bar{f}+\text { c.c. })
$$

where $Y_{f} \equiv m_{f} / v$ (with $v \approx 174 \mathrm{GeV}$ ) are real positive Yukawa couplings for the mass eigenstate fermions $f=u, c, t$ and $d, s, b$ and $e, \mu, \tau$. These couplings imply the Feynman rules in Figure 3.3 . 

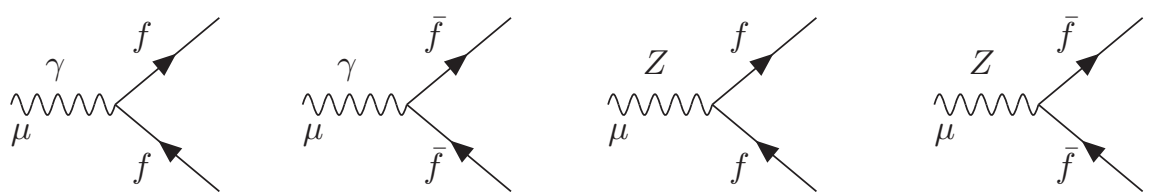

$$
i e Q_{f} \bar{\sigma}_{\mu}
$$

$-i e Q_{f} \bar{\sigma}_{\mu}$

$i \frac{g}{c_{W}}\left(T_{3}^{f}-s_{W}^{2} Q_{f}\right) \bar{\sigma}_{\mu}$

$$
i \frac{g s_{W}^{2}}{c_{W}} Q_{f} \bar{\sigma}_{\mu}
$$
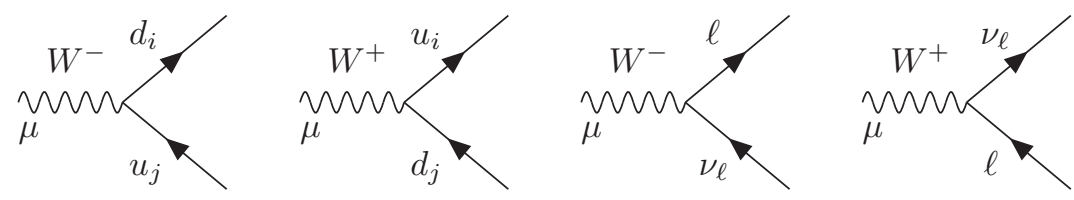

$\frac{i}{\sqrt{2}} g\left[\boldsymbol{K}^{\dagger}\right]_{i}{ }^{j} \bar{\sigma}_{\mu}$

$\frac{i}{\sqrt{2}} g[\boldsymbol{K}]_{i}^{j} \bar{\sigma}_{\mu}$

$\frac{i}{\sqrt{2}} g \bar{\sigma}_{\mu}$

$\frac{i}{\sqrt{2}} g \bar{\sigma}_{\mu}$

Fig. 3.1: Feynman rules for the 2-component fermion interactions with electroweak gauge bosons in the Standard Model. For each rule, there is a corresponding one with lowered spinor indices, obtained by $\bar{\sigma}_{\mu} \rightarrow-\sigma_{\mu}$.
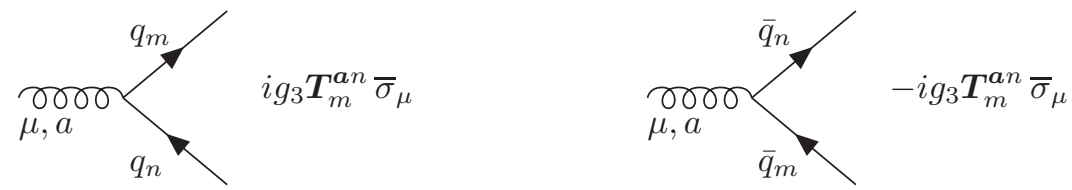

Fig. 3.2: Fermionic Feynman rules for QCD that involve the gluon, with $q=u, d, c, s, t, b$. Lowered (raised) indices $m, n$ correspond to the fundamental (anti-fundamental) representation of $S U(3)_{c}$. For each rule, there is a corresponding one with $\bar{\sigma}_{\mu} \rightarrow-\sigma_{\mu}$.

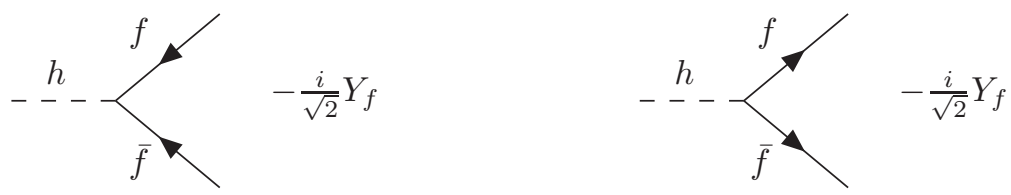

Fig. 3.3: Feynman rules for the Standard Model Higgs boson interactions with quarks and leptons.

\section{Fermion Feynman rules in the Minimal Supersymmetric Standard Model}

Next let us consider the Feynman rules for the 2-component fermions in the MSSM. These can be derived from the rules for writing down supersymmetric Lagrangians in the prerequisite, ref. [11].

We will begin with the vector boson interactions with fermions. For the quarks and leptons, the rules are exactly the same as in the Standard Model, see Figures [3.1] and 3.2. The gluino is also easy, because it in the adjoint rep of $S U(3)_{c}$ and does not mix with any other particle. The gluon-gluino-gluino interaction Feynman rule is shown in Figure 4.1.

Neutralinos and charginos have mixing, which makes their mass eigenstates differ 


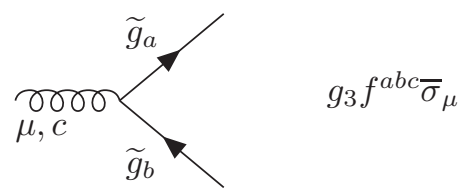

Fig. 4.1: Feynman rule for the gluon-gluino-gluino coupling in the MSSM. There is another rule with $\bar{\sigma}_{\mu} \rightarrow-\sigma_{\mu}$.

from the gauge eigenstates. To obtain the Feynman rules, consider first the mass matrices in the gauge eigenstate bases:

$$
\begin{aligned}
& M_{\psi^{0}}=\left(\begin{array}{cccc}
M_{1} & 0 & -g^{\prime} v_{d} / \sqrt{2} & g^{\prime} v_{u} / \sqrt{2} \\
0 & M_{2} & g v_{d} / \sqrt{2} & -g v_{u} / \sqrt{2} \\
-g^{\prime} v_{d} / \sqrt{2} & g v_{d} / \sqrt{2} & 0 & -\mu \\
g^{\prime} v_{u} / \sqrt{2} & -g v_{u} / \sqrt{2} & -\mu & 0
\end{array}\right), \\
& M_{\psi^{ \pm}}=\left(\begin{array}{cc}
M_{2} & g v_{u} \\
g v_{d} & \mu
\end{array}\right) .
\end{aligned}
$$

As discussed in ref. [11, these can be diagonalized by unitary matrices $N$ for neutralinos and $U, V$ for charginos, according to:

$$
\begin{aligned}
& N^{*} M_{\psi^{0}} N^{-1}=\operatorname{diag}\left(m_{\widetilde{N}_{1}}, m_{\widetilde{N}_{2}}, m_{\widetilde{N}_{3}}, m_{\widetilde{N}_{4}}\right), \\
& U^{*} M_{\psi^{ \pm}} V^{-1}=\operatorname{diag}\left(m_{\widetilde{C}_{1}}, m_{\widetilde{C}_{2}}\right) .
\end{aligned}
$$

Now, following ref. [1], we define:

$$
\begin{aligned}
O_{i j}^{L} & =-\frac{1}{\sqrt{2}} N_{i 4} V_{j 2}^{*}+N_{i 2} V_{j 1}^{*}, \\
O_{i j}^{R} & =\frac{1}{\sqrt{2}} N_{i 3}^{*} U_{j 2}+N_{i 2}^{*} U_{j 1}, \\
O_{i j}^{\prime L} & =-V_{i 1} V_{j 1}^{*}-\frac{1}{2} V_{i 2} V_{j 2}^{*}+\delta_{i j} s_{W}^{2}, \\
O_{i j}^{\prime R} & =-U_{i 1}^{*} U_{j 1}-\frac{1}{2} U_{i 2}^{*} U_{j 2}+\delta_{i j} s_{W}^{2}, \\
O_{i j}^{\prime \prime} & =-O_{j i}^{\prime \prime}=\frac{1}{2}\left(N_{i 4} N_{j 4}^{*}-N_{i 3} N_{j 3}^{*}\right) .
\end{aligned}
$$

In terms of these coupling matrices, the Feynman rules for vector boson interactions with charginos and neutralinos are as shown in Figure 4.2. That concludes the vector interactions with fermions in the MSSM.

The quark and lepton interactions with Higgs bosons are different in the MSSM than in the Standard Model, because we start with two Higgs doublet fields $H_{u}=\left(H_{u}^{+}, H_{u}^{0}\right)$ and $H_{d}=\left(H_{d}^{0}, H_{d}^{-}\right)$rather than one. To obtain Feynman rules involving the Higgs boson mass eigenstates, it is useful to write those gauge eigenstates in terms of mass eigenstate complex charged fields $\phi^{ \pm}=\left(H^{ \pm}, G^{ \pm}\right)$, and real neutral fields $\phi^{0}=\left(h^{0}, H^{0}, A^{0}, G^{0}\right)$, by 

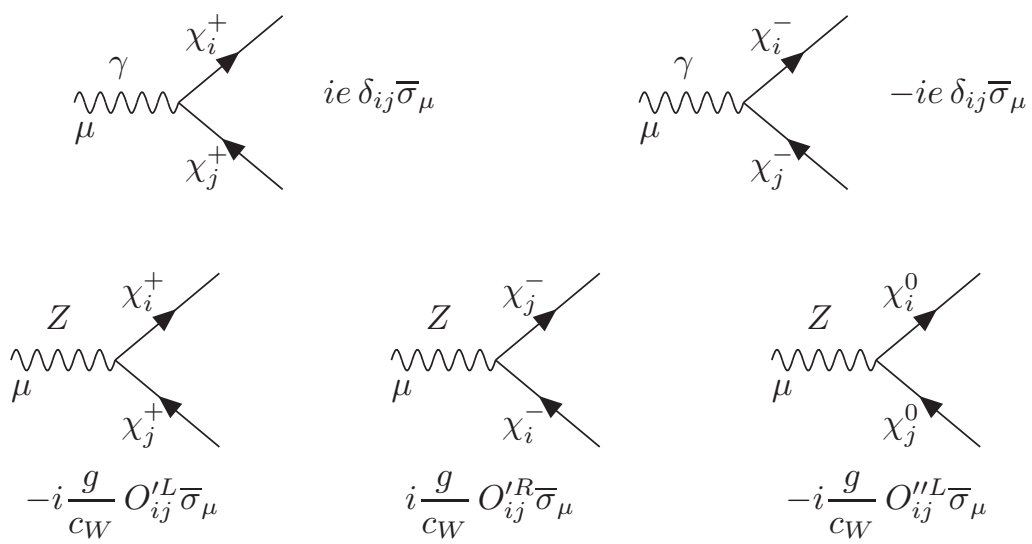

$-i \frac{g}{c_{W}} O_{i j}^{\prime \prime L} \bar{\sigma}_{\mu}$
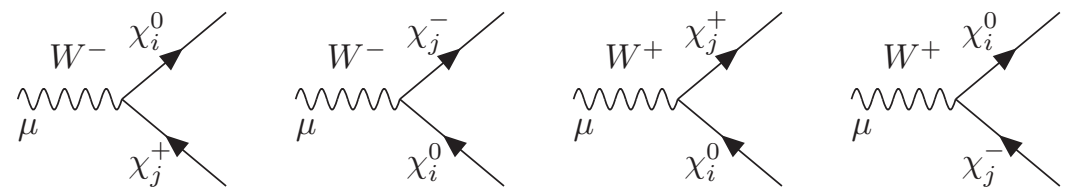

$$
-i g O_{i j}^{L} \bar{\sigma}_{\mu}
$$

$i g O_{i j}^{R} \bar{\sigma}_{\mu}$

$-i g O_{i j}^{L *} \bar{\sigma}_{\mu}$

$i g O_{i j}^{R *} \bar{\sigma}_{\mu}$

Fig. 4.2: Feynman rules for the chargino and neutralino interactions with electroweak vector bosons in the MSSM. The coupling matrices $O^{L}, O^{R}, O^{\prime L}, O^{\prime R}$ and $O^{\prime \prime L}$ are defined in eqs. (4.5)-(4.9). For each rule, there is a corresponding one obtained by $\bar{\sigma}_{\mu} \rightarrow-\sigma_{\mu}$.

expanding around VEVs $v_{u}$ and $v_{d}$ :

$$
\begin{aligned}
H_{u}^{0} & =v_{u}+\frac{1}{\sqrt{2}} \sum_{\phi^{0}} k_{u \phi^{0}} \phi^{0}, & H_{u}^{ \pm} & =\sum_{\phi^{ \pm}} k_{u \phi^{ \pm}} \phi^{ \pm}, \\
H_{d}^{0} & =v_{d}+\frac{1}{\sqrt{2}} \sum_{\phi^{0}} k_{d \phi^{0}} \phi^{0}, & H_{d}^{ \pm} & =\sum_{\phi^{ \pm}} k_{d \phi^{ \pm}} \phi^{ \pm} .
\end{aligned}
$$

Here $\phi^{-} \equiv\left(\phi^{+}\right)^{*}$, and $G^{0}$ and $G^{ \pm}$are the would-be Goldstone bosons, which become the longitudinal components of the $Z$ and $W$ bosons. The VEVs are normalized so that $v_{u}^{2}+v_{d}^{2} \approx(174 \mathrm{GeV})^{2}$, and their ratio is defined to be

$$
v_{u} / v_{d} \equiv \tan \beta .
$$

The mixing parameters can be written:

$$
\begin{aligned}
& k_{u \phi^{ \pm}}=\left(\begin{array}{ll}
\cos \beta_{ \pm}, & \sin \beta_{ \pm}
\end{array}\right), \\
& k_{d \phi^{ \pm}}=\left(\begin{array}{ll}
\sin \beta_{ \pm}, & -\cos \beta_{ \pm}
\end{array}\right),
\end{aligned}
$$

for $\phi^{ \pm}=\left(H^{ \pm}, G^{ \pm}\right)$, and

$$
\begin{aligned}
& k_{u \phi^{0}}=\left(\cos \alpha, \sin \alpha, i \cos \beta_{0}, i \sin \beta_{0}\right), \\
& k_{d \phi^{0}}=\left(-\sin \alpha, \cos \alpha, i \sin \beta_{0},-i \cos \beta_{0}\right),
\end{aligned}
$$

for $\phi^{0}=\left(h^{0}, H^{0}, A^{0}, G^{0}\right)$. If the VEVs $v_{u}$ and $v_{d}$ are chosen to minimize the tree-level scalar potential, then one can show that $\beta_{ \pm}=\beta_{0}=\beta$, and the interaction couplings are 


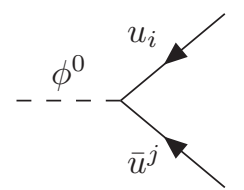

$-\frac{i}{\sqrt{2}} Y_{u i} k_{u \phi^{0}} \delta_{j}^{i}$

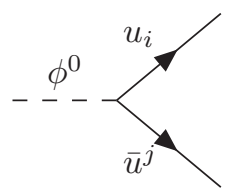

$-\frac{i}{\sqrt{2}} Y_{u i} k_{u \phi^{0}}^{*} \delta_{i}^{j}$

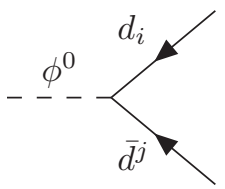

$-\frac{i}{\sqrt{2}} Y_{d i} k_{d \phi^{0}} \delta_{j}^{i}$

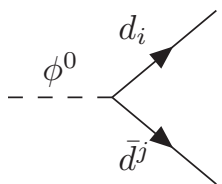

$-\frac{i}{\sqrt{2}} Y_{d i} k_{d \phi^{0}}^{*} \delta_{i}^{j}$

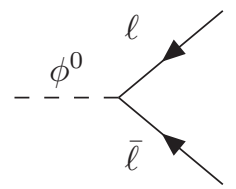

$-\frac{i}{\sqrt{2}} Y_{\ell} k_{d \phi^{0}}$

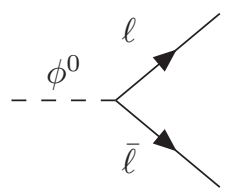

$-\frac{i}{\sqrt{2}} Y_{\ell} k_{d \phi^{0}}^{*}$

Fig. 4.3: Feynman rules for the interactions of neutral Higgs bosons $\phi^{0}=\left(h^{0}, H^{0}, A^{0}, G^{0}\right)$ with fermion-antifermion pairs in the MSSM. The repeated index $i$ is not summed.

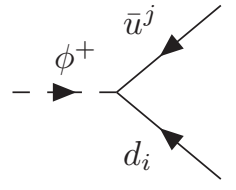

$i Y_{u j}[\boldsymbol{K}]_{j}{ }^{i} k_{u \phi^{ \pm}}$

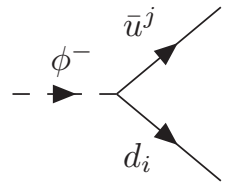

$i Y_{u j}\left[\boldsymbol{K}^{\dagger}\right]_{i}{ }^{j} k_{u \phi^{ \pm}}$

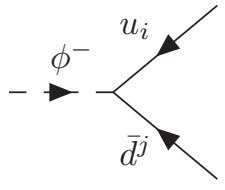

$i Y_{d j}\left[\boldsymbol{K}^{\dagger}\right]_{j}{ }^{i} k_{d \phi^{ \pm}}$

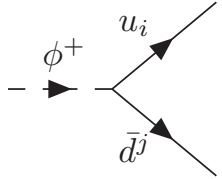

$i Y_{d j}[\boldsymbol{K}]_{i}{ }^{j} k_{d \phi^{ \pm}}$

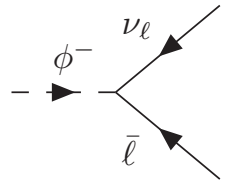

$i Y_{\ell} k_{d \phi^{ \pm}}$

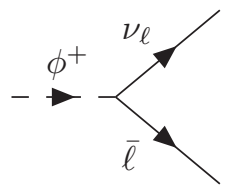

$i Y_{\ell} k_{d \phi^{ \pm}}$

Fig. 4.4: Feynman rules for the interactions of charged Higgs bosons $\phi^{ \pm}=\left(H^{ \pm}, G^{ \pm}\right)$with fermionantifermion pairs in the MSSM. The meaning of the arrows on the scalar lines is that the $\phi^{ \pm}$line carry charges \pm 1 into the vertex. The repeated index $j$ is not summed.

often written making that assumption. $\mathrm{c}$ Also, $\alpha$ is an independent mixing angle. In the decoupling limit where $M_{h^{0}} \ll M_{H^{ \pm}}, M_{A^{0}}, M_{H^{0}}$, one has $\alpha \approx \beta-\pi / 2$.

Using the above notation, the interactions of quarks and leptons with the neutral Higgs bosons are as shown in Figures 4.3 and 4.4. The rules for Higgs boson couplings

\footnotetext{
${ }^{\mathrm{c}}$ However, $v_{u}$ and $v_{d}$ need not be the minima of the tree-level scalar potential; sometimes it is more useful and accurate to take them to be minima of the effective potential, suitably approximated at one-loop or two-loop order. More generally, one can expand around any VEVs $v_{u}$ and $v_{d}$, at the cost of including suitable tadpole couplings. Then $\beta_{ \pm}, \beta_{0}$, and $\beta$ are all different. Indeed, this is what one must do when computing the effective potential as a function of the VEVs, because in that case one certainly does not want to consider the VEVs as fixed. This is why we distinguish between $\beta_{ \pm}$ and $\beta_{0}$ and $\beta$.
} 

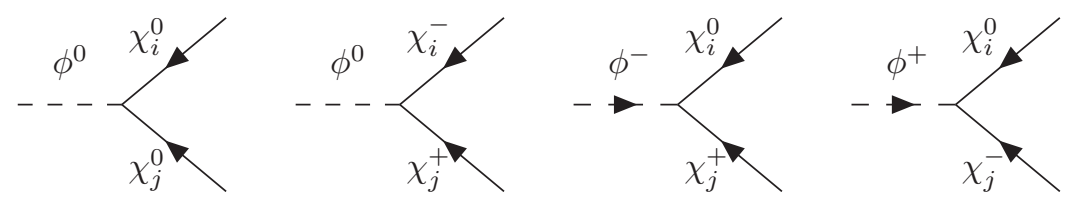

$-i Y^{\phi^{0} \chi_{i}^{0} \chi_{j}^{0}}$

$-i Y^{\phi^{0} \chi_{i}^{-} \chi_{j}^{+}}$

$-i Y^{\phi^{-}} \chi_{i}^{0} \chi_{j}^{+}$

$-i Y^{\phi^{+} \chi_{i}^{0} \chi_{j}^{-}}$

Fig. 4.5: Feynman rules for the chargino and neutralino interactions with Higgs bosons in the MSSM. The couplings are defined in eqs. (4.5)-(4.9). For each rule, there is a corresponding one with all arrows reversed, and the $Y$ coupling (without the explicit $i$ ) replaced by its complex conjugate.

to neutralinos and charginos are shown in Figure 4.5, in terms of

$$
\begin{aligned}
Y^{\phi^{0} \chi_{i}^{0} \chi_{j}^{0}} & =\frac{1}{2}\left(k_{d \phi^{0}}^{*} N_{i 3}^{*}-k_{u \phi^{0}}^{*} N_{i 4}^{*}\right)\left(g N_{j 2}^{*}-g^{\prime} N_{j 1}^{*}\right)+(i \leftrightarrow j), \\
Y^{\phi^{0} \chi_{i}^{-} \chi_{j}^{+}} & =\frac{g}{\sqrt{2}}\left(k_{u \phi^{0}}^{*} U_{i 1}^{*} V_{j 2}^{*}+k_{d \phi^{0}}^{*} U_{i 2}^{*} V_{j 1}^{*}\right), \\
Y^{\phi^{+} \chi_{i}^{0} \chi_{j}^{-}} & =k_{d \phi^{ \pm}}\left[g\left(N_{i 3}^{*} U_{j 1}^{*}-\frac{1}{\sqrt{2}} N_{i 2}^{*} U_{j 2}^{*}\right)-\frac{g^{\prime}}{\sqrt{2}} N_{i 1}^{*} U_{j 2}^{*}\right], \\
Y^{\phi^{-} \chi_{i}^{0} \chi_{j}^{+}} & =k_{u \phi^{ \pm}}\left[g\left(N_{i 4}^{*} V_{j 1}^{*}+\frac{1}{\sqrt{2}} N_{i 2}^{*} V_{j 2}^{*}\right)+\frac{g^{\prime}}{\sqrt{2}} N_{i 1}^{*} V_{j 2}^{*}\right],
\end{aligned}
$$

for $\phi^{0}=h^{0}, H^{0}, A^{0}, G^{0}$ and $\phi^{ \pm}=H^{ \pm}, G^{ \pm}$.

Feynman rules for sfermion-fermion in interactions with charginos, neutralinos, and the gluino in the MSSM appear in Figures 4.6, 4.7, and 4.8, respectively. In these rules, the Standard Model quarks and leptons are assumed to be in the mass eigenstate bases, and the squarks and sleptons are assumed to be in the basis defined by superpartners of the fermion mass eigenstates.

However, in principle all sfermions with a given electric charge can mix with each other. There is a popular, and perhaps phenomenologically and theoretically favored, approximation in which only the sfermions of the third family have significant mixing. For $f=t, b, \tau$, one can then write the relationship between the gauge eigenstates $\widetilde{f}_{L}, \widetilde{f}_{R}$ and the mass eigenstates $\tilde{f}_{1}, \widetilde{f}_{2}$ as

$$
\left(\begin{array}{c}
\widetilde{f}_{R} \\
\widetilde{f}_{L}
\end{array}\right)=X_{\widetilde{f}}\left(\begin{array}{c}
\widetilde{f}_{1} \\
\widetilde{f}_{2}
\end{array}\right), \quad X_{\widetilde{f}} \equiv\left(\begin{array}{c}
R_{\widetilde{f}_{1}} R_{\widetilde{f}_{2}} \\
L_{\widetilde{f}_{1}} L_{\widetilde{f}_{2}}
\end{array}\right),
$$

where $X$ is a $2 \times 2$ unitary matrix.

[One can choose $R_{\widetilde{f}_{1}}=L_{\widetilde{f}_{2}}^{*}=c_{\widetilde{f}}$, and $L_{\widetilde{f}_{1}}=-R_{\widetilde{f}_{2}}^{*}=s_{\widetilde{f}}$, with $\left|c_{\widetilde{f}}\right|^{2}+\left|s_{\widetilde{f}}\right|^{2}=1$. If there is no CP violation, then $c_{\tilde{f}}$ and $s_{\tilde{f}}$ can be taken real, and they are the cosine and sine of a sfermion mixing angle. This convention for $c_{\tilde{f}}, s_{\widetilde{f}}$ has the nice property that for zero mixing angle, $\tilde{f}_{1}=\widetilde{f}_{R}$ and $\widetilde{f}_{2}=\widetilde{f}_{L}$. Various other conventions are found in the literature. We use $R_{\widetilde{f}_{i}}$ and $L_{\widetilde{f}_{i}}$ in the Feynman rules, rather than $c_{\widetilde{f}}$ and $s_{\widetilde{f}}$, to make it easier to compare to your favorite mixing angle convention using eq. (4.21).] 

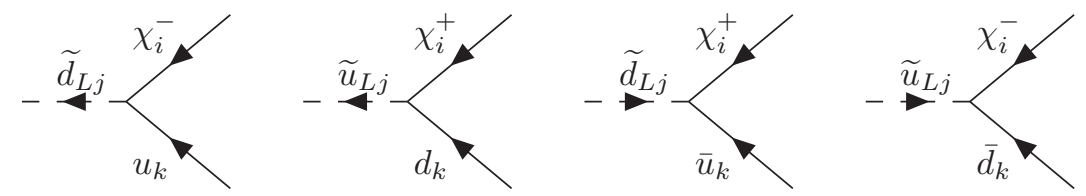

$-i g U_{i 1}^{*}\left[\boldsymbol{K}^{\dagger}\right]_{j}$

$-i g V_{i 1}^{*}[\boldsymbol{K}]_{j}{ }^{k}$

$i V_{i 2}^{*}[\boldsymbol{K}]_{k}^{j} Y_{u k}$

$i U_{i 2}^{*}\left[\boldsymbol{K}^{\dagger}\right]_{k}^{j} Y_{d j}$
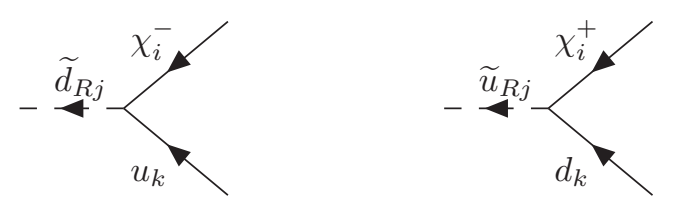

$i U_{i 2}^{*}\left[\boldsymbol{K}^{\dagger}\right]_{j}{ }^{k} Y_{d j}$

$i V_{i 2}^{*}[\boldsymbol{K}]_{j}{ }^{k} Y_{u j}$
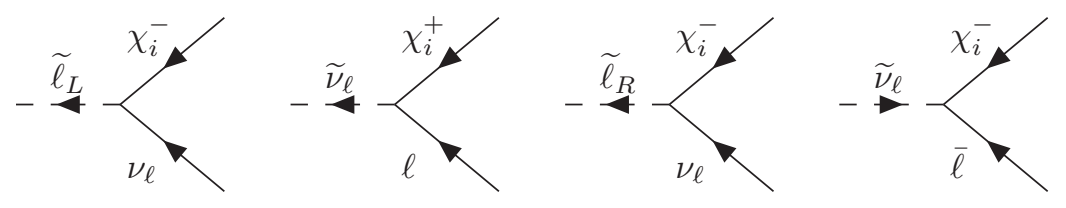

$-i g U_{i 1}^{*}$

$-i g V_{i 1}^{*}$

$i U_{i 2}^{*} Y_{\ell}$

$i U_{i 2}^{*} Y_{\ell}$

Fig. 4.6: Feynman rules for charginos interactions with fermion/sfermion pairs in the MSSM. The fermions are taken to be in a mass eigenstate basis, and the sfermions are in a basis whose elements are the supersymmetric partners of the fermions. This is usually considered to be a good approximation for the squarks and sleptons of the first two families. For each rule, there is a corresponding one with all arrows reversed and the coupling (without the explicit $i$ ) replaced by its complex conjugate.

The resulting Feynman rules for chargino, neutralino, and gluino interactions with third-family squarks and sleptons that mix within each generation are shown in Figures 4.9, 4.10, and 4.11. The neutralino interaction rules in Figure 4.10 make use of the following couplings:

$$
\begin{aligned}
& Y^{\widetilde{t}_{j}^{*} t \chi_{i}^{0}}=Y_{t} N_{i 4}^{*} R_{\widetilde{t}_{j}}^{*}+\frac{1}{\sqrt{2}}\left(g N_{i 2}^{*}+\frac{1}{3} g^{\prime} N_{i 1}^{*}\right) L_{\widetilde{t}_{j}}^{*}, \\
& Y^{\widetilde{t}_{j} \bar{t} \chi_{i}^{0}}=Y_{t} N_{i 4}^{*} L_{\widetilde{t}_{j}}-\frac{2 \sqrt{2}}{3} g^{\prime} N_{i 1}^{*} R_{\widetilde{t}_{j}}, \\
& Y^{\widetilde{b_{j}^{*}} b \chi_{i}^{0}}=Y_{b} N_{i 3}^{*} R_{\widetilde{b}_{j}}^{*}+\frac{1}{\sqrt{2}}\left(-g N_{i 2}^{*}+\frac{1}{3} g^{\prime} N_{i 1}^{*}\right) L_{\widetilde{b}_{j}}^{*}, \\
& Y^{\widetilde{b_{j}} \bar{b} \chi_{i}^{0}}=Y_{b} N_{i 3}^{*} L_{\widetilde{b}_{j}}+\frac{\sqrt{2}}{3} g^{\prime} N_{i 1}^{*} R_{\widetilde{b}_{j}}, \\
& Y^{\widetilde{\tau}_{j}^{*} \tau \chi_{i}^{0}}=Y_{\tau} N_{i 3}^{*} R_{\widetilde{\tau}_{j}}^{*}-\frac{1}{\sqrt{2}}\left(g N_{i 2}^{*}+g^{\prime} N_{i 1}^{*}\right) L_{\widetilde{\tau}_{j}}^{*} . \\
& Y^{\widetilde{\tau}_{j} \bar{\tau} \chi_{i}^{0}}=Y_{\tau} N_{i 3}^{*} L_{\widetilde{\tau}_{j}}+\sqrt{2} g^{\prime} N_{i 1}^{*} R_{\widetilde{\tau}_{j}} .
\end{aligned}
$$

The rules in Figures 4.9, 4.10, and 4.11 can be obtained from the preceding three diagrams by simply taking the appropriate linear combinations of Feynman rules for 


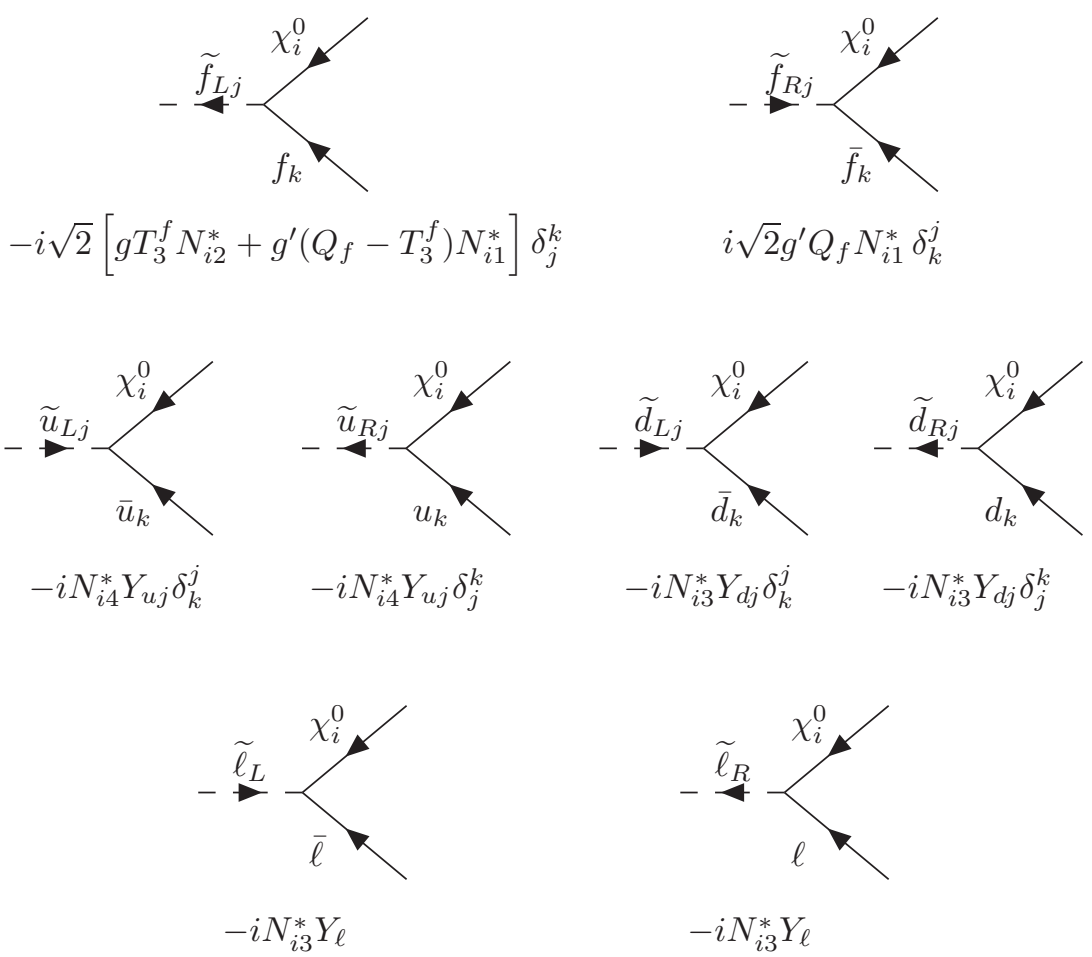

Fig. 4.7: Feynman rules for the interactions of neutralinos with first and second family fermion/sfermion pairs in the MSSM. The comments on Figure 4.6 also apply here.
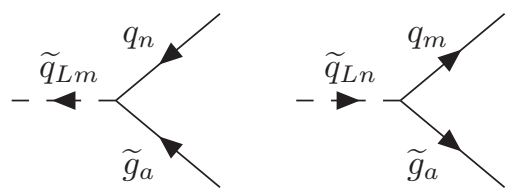

$$
-i \sqrt{2} g_{3} \boldsymbol{T}_{m}^{a n}
$$

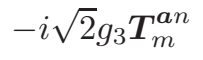

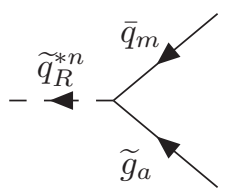

$i \sqrt{2} g_{3} \boldsymbol{T}_{m}^{a n}$

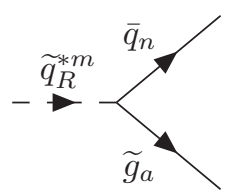

$i \sqrt{2} g_{3} \boldsymbol{T}_{m}^{a n}$

Fig. 4.8: Feynman rules for gluino interactions with first and second family quark/squark pairs in the MSSM. The indices $m, n$ are for the fundamental representation of $S U(3)_{c}$, and $a$ is an adjoint representation index. The comments on Figure 4.6 also apply here.

unmixed squarks and sleptons. Conversely, for the charged sfermions of the first two families, $(\widetilde{f}=\widetilde{u}, \widetilde{d}, \widetilde{c}, \widetilde{s}, \widetilde{e}, \widetilde{\mu})$, one can use the same notation as in Figures 4.9, 4.10, and 4.11, and take $Y_{f}=0$ and $L_{\widetilde{f}_{2}}=R_{\widetilde{f}_{1}}=1$ and $L_{\widetilde{f}_{1}}=R_{\widetilde{f}_{2}}=0$. 

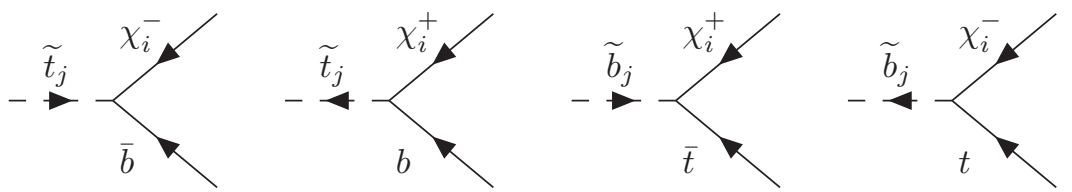

$i Y_{b} U_{i 2}^{*} L_{\widetilde{t}_{j}} \quad i\left(Y_{t} V_{i 2}^{*} R_{\widetilde{t}_{j}}^{*}-g V_{i 1}^{*} L_{\widetilde{t}_{j}}^{*}\right)$

$i Y_{t} V_{i 2}^{*} L_{\widetilde{b}_{j}} \quad i\left(Y_{b} U_{i 2}^{*} R_{\widetilde{b}_{j}}^{*}-g U_{i 1}^{*} L_{\widetilde{b}_{j}}^{*}\right)$

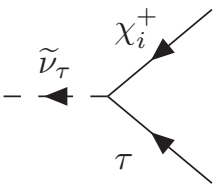

$-i g V_{i 1}^{*}$

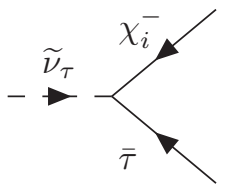

$i Y_{\tau} U_{i 2}^{*}$

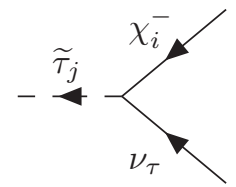

$i\left(Y_{\tau} U_{i 2}^{*} R_{\widetilde{\tau}_{j}}^{*}-g U_{i 1}^{*} L_{\widetilde{\tau}_{j}}^{*}\right)$

Fig. 4.9: Feynman rules for chargino interactions with third-family fermion/sfermion pairs. The fermions are taken to be in a mass eigenstate basis, and the sfermions are in the mass eigenstate basis of eq. (4.21). For each rule, there is a corresponding one with all arrows reversed and the coupling (without the explicit $i$ ) replaced by its complex conjugate.

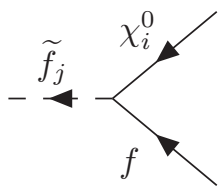

$-i Y^{\widetilde{f}_{j}^{*} f \chi_{i}^{0}}$

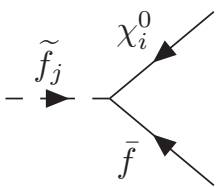

$-i Y^{\tilde{f}} \bar{f} \chi_{i}^{0}$

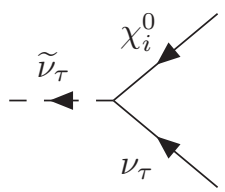

$-\frac{i}{\sqrt{2}}\left(g N_{i 2}^{*}-g^{\prime} N_{i 1}^{*}\right)$

Fig. 4.10: Feynman rules for neutralino interactions with third-family fermion/sfermion pairs in the MSSM. Here $f=t, b, \tau$, with couplings $Y^{\widetilde{f}_{j}^{*}} f \chi_{i}^{0}$ and $Y^{\widetilde{f}_{j}} \bar{f} \chi_{i}^{0}$ given in eqs. (4.22)-(4.27). The comments on Figure 4.9 also apply here.
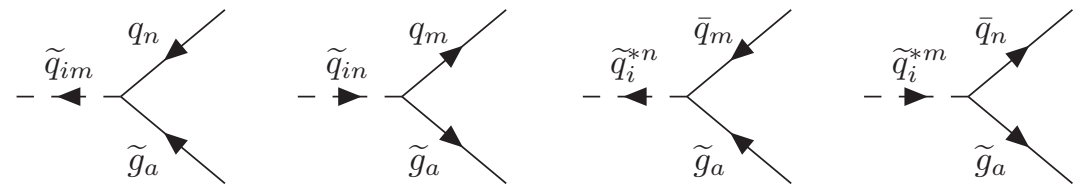

$$
-i \sqrt{2} g_{3} L_{\widetilde{q}_{i}}^{*} \boldsymbol{T}_{m}^{a n}
$$

$$
-i \sqrt{2} g_{3} L_{\widetilde{q}_{i}} \boldsymbol{T}_{m}^{a n}
$$

$i \sqrt{2} g_{3} R_{\widetilde{q}_{i}} \boldsymbol{T}_{m}^{a n}$

$i \sqrt{2} g_{3} R_{\widetilde{q}_{i}}^{*} \boldsymbol{T}_{m}^{a n}$

Fig. 4.11: Feynman rules for gluino interactions with third-family quark/squark pairs in the MSSM. The indices $m, n$ are for the fundamental representation of $S U(3)_{c}$, and $a$ is an adjoint representation index. The index $i=1,2$ runs over the mass eigenstates. The comments on Figure 4.10 also apply here. 


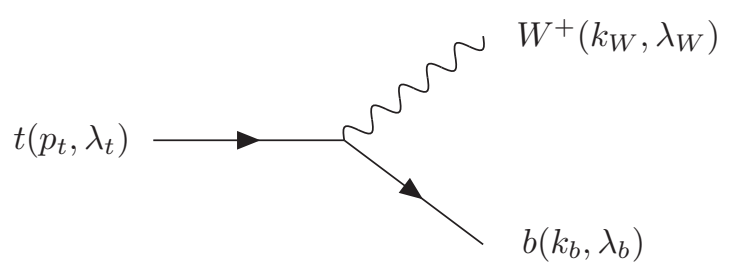

Fig. 5.1.1: The Feynman diagram for $t \rightarrow b W^{+}$at tree level.

\section{Examples}

\subsection{Top quark decay: $t \rightarrow b W^{+}$}

We begin by calculating the decay width of a top quark into a bottom quark and $W^{+}$ vector boson. Let the four-momenta and helicities of these particle be $\left(p_{t}, \lambda_{t}\right),\left(k_{b}, \lambda_{b}\right)$ and $\left(k_{W}, \lambda_{W}\right)$, respectively. Then $p_{t}^{2}=-m_{t}^{2}$ and $k_{b}^{2}=-m_{b}^{2}$ and $k_{W}^{2}=-m_{W}^{2}$ and

$$
\begin{aligned}
2 p_{t} \cdot k_{W} & =-m_{t}^{2}+m_{b}^{2}-m_{W}^{2}, \\
2 p_{t} \cdot k_{b} & =-m_{t}^{2}-m_{b}^{2}+m_{W}^{2}, \\
2 k_{W} \cdot k_{b} & =-m_{t}^{2}+m_{b}^{2}+m_{W}^{2} .
\end{aligned}
$$

Because only left-handed top quarks couple to the $W$ boson, the only Feynman diagram for $t \rightarrow b W^{+}$is the one shown in Fig. 5.1.1. The corresponding amplitude can be read off of the fifth Feynman rule of Fig. 3.1. Here the initial state top quark is a 2-component field $t$ going into the vertex and the final state bottom quark is created by a 2-component field $b^{\dagger}$. Therefore the amplitude is given by, using $\boldsymbol{K}_{3}{ }^{3}=V_{t b}$ :

$$
i \mathcal{M}=i \frac{g}{\sqrt{2}} V_{t b}^{*} \varepsilon_{\mu}^{*} x_{b}^{\dagger} \bar{\sigma}^{\mu} x_{t}
$$

where $\varepsilon_{\mu}^{*} \equiv \varepsilon_{\mu}\left(k_{W}, \lambda_{W}\right)^{*}$ is the polarization vector of the $W^{+}$, and $x_{b}^{\dagger} \equiv x^{\dagger}\left(\overrightarrow{\boldsymbol{k}}_{b}, \lambda_{b}\right)$ and $x_{t} \equiv x\left(\vec{p}_{t}, \lambda_{t}\right)$ are the external state wave functions for the bottom and top quark. Squaring this amplitude using eq. (2.1.32) yields:

$$
|\mathcal{M}|^{2}=\frac{g^{2}}{2}\left|V_{t b}\right|^{2} \varepsilon_{\mu}^{*} \varepsilon_{\nu}\left(x_{b}^{\dagger} \bar{\sigma}^{\mu} x_{t}\right)\left(x_{t}^{\dagger} \bar{\sigma}^{\nu} x_{b}\right) .
$$

Next, we can average over the top quark spin polarizations using eq. (2.3.3):

$$
\frac{1}{2} \sum_{\lambda_{t}}|\mathcal{M}|^{2}=\frac{g^{2}}{4}\left|V_{t b}\right|^{2} \varepsilon_{\mu}^{*} \varepsilon_{\nu} x_{b}^{\dagger} \bar{\sigma}^{\mu} p_{t} \cdot \sigma \bar{\sigma}^{\nu} x_{b} .
$$

Summing over the bottom quark spin polarizations in the same way yields a trace over spinor indices:

$$
\begin{aligned}
& \frac{1}{2} \sum_{\lambda_{t}, \lambda_{b}}|\mathcal{M}|^{2}=\frac{g^{2}}{4}\left|V_{t b}\right|^{2} \varepsilon_{\mu}^{*} \varepsilon_{\nu} \operatorname{Tr}\left[\bar{\sigma}^{\mu} p_{t} \cdot \sigma \bar{\sigma}^{\nu} k_{b} \cdot \sigma\right] \\
& \quad=\frac{g^{2}}{2}\left|V_{t b}\right|^{2} \varepsilon_{\mu}^{*} \varepsilon_{\nu}\left(p_{t}^{\mu} k_{b}^{\nu}+k_{b}^{\mu} p_{t}^{\nu}-g^{\mu \nu} p_{t} \cdot k_{b}-i \epsilon^{\mu \rho \nu \kappa} p_{t \rho} k_{b \kappa}\right),
\end{aligned}
$$


where we have used eq. (2.1.45). From here, the calculation is unaffected by the treatment of the fermionic Feynman rules. One sums over the $W^{+}$polarizations according to:

$$
\sum_{\lambda_{W}} \varepsilon_{\mu}^{*} \varepsilon_{\nu}=g_{\mu \nu}+\left(k_{W}\right)_{\mu}\left(k_{W}\right)_{\nu} / m_{W}^{2}
$$

The end result is:

$$
\frac{1}{2} \sum_{\text {spins }}|\mathcal{M}|^{2}=\frac{g^{2}}{2}\left|V_{t b}\right|^{2}\left[-p_{t} \cdot k_{b}+2\left(p_{t} \cdot k_{W}\right)\left(k_{b} \cdot k_{W}\right) / m_{W}^{2}\right] .
$$

After performing the phase space integration, one obtains:

$$
\begin{aligned}
\Gamma(t & \left.\rightarrow b W^{+}\right)=\frac{\left|V_{t b}\right|^{2}}{16 \pi m_{t}^{3}} \lambda^{1 / 2}\left(m_{t}^{2}, m_{W}^{2}, m_{b}^{2}\right)\left(\frac{1}{2} \sum_{\text {spins }}|\mathcal{M}|^{2}\right) \\
= & \frac{g^{2}\left|V_{t b}\right|^{2}}{64 \pi m_{W}^{2} m_{t}^{3}} \lambda^{1 / 2}\left(m_{t}^{2}, m_{W}^{2}, m_{b}^{2}\right)\left[\left(m_{t}^{2}+2 m_{W}^{2}\right)\left(m_{t}^{2}-m_{W}^{2}\right)\right. \\
& \left.+m_{b}^{2}\left(m_{W}^{2}-2 m_{t}^{2}\right)+m_{b}^{4}\right],
\end{aligned}
$$

where the kinematic triangle function $\lambda^{1 / 2}$ is defined as usual by:

$$
\lambda(x, y, z) \equiv x^{2}+y^{2}+z^{2}-2 x y-2 x z-2 y z .
$$

In the approximation $m_{b} \ll m_{W}, m_{t}$, one obtains the well-known result

$$
\Gamma\left(t \rightarrow b W^{+}\right)=\frac{m_{t} g^{2}\left|V_{t b}\right|^{2}}{64 \pi}\left(2+\frac{m_{t}^{2}}{m_{W}^{2}}\right)\left(1-\frac{m_{W}^{2}}{m_{t}^{2}}\right)^{2},
$$

exhibiting the Nambu-Goldstone enhancement factor $\left(m_{t}^{2} / m_{W}^{2}\right)$ for the longitudinal $W$ contribution compared to the two transverse $W$ contributions.

\section{2. $Z^{0}$ vector boson decay: $Z^{0} \rightarrow f \bar{f}$}

Consider the partial decay width of the $Z^{0}$ boson into a Standard Model fermionantifermion pair. There are two contributing Feynman diagrams, shown in Fig. 5.2.1. In diagram (a), the fermion particle $f$ in the final state is created by a 2-component field $f$ in the Feynman rule, and the antifermion particle $\bar{f}$ by a 2-component field $f^{\dagger}$.

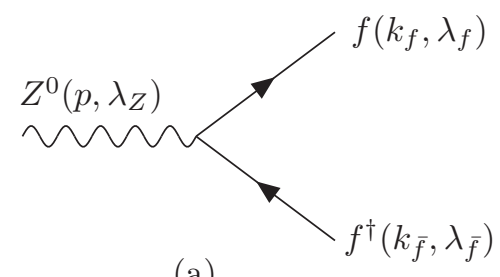

(a)

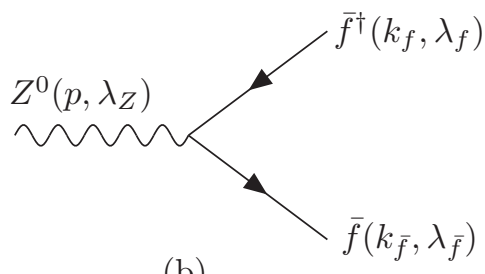

(b)

Fig. 5.2.1: The Feynman diagrams for $Z^{0}$ decay into a fermion-antifermion pair. Fermion lines are labeled according to the 2-component fermion field labeling convention established in Section 2.6 
In diagram (b), the fermion particle $f$ in the final state is created by a 2 -component field $\bar{f}$, and the antifermion particle $\bar{f}$ by a 2 -component field $\bar{f}^{\dagger}$. Denote the initial $Z^{0}$ four-momentum and helicity $\left(p, \lambda_{Z}\right)$ and the final state fermion $(f)$ and antifermion $(\bar{f})$ momentum and helicities $\left(k_{f}, \lambda_{f}\right)$ and $\left(k_{\bar{f}}, \lambda_{\bar{f}}\right)$, respectively. Then, $k_{f}^{2}=k_{\bar{f}}^{2}=-m_{f}^{2}$ and $p^{2}=-m_{Z}^{2}$, and

$$
\begin{aligned}
& k_{f} \cdot k_{\bar{f}}=-\frac{1}{2} m_{Z}^{2}+m_{f}^{2}, \\
& p \cdot k_{f}=p \cdot k_{\bar{f}}=-\frac{1}{2} m_{Z}^{2} .
\end{aligned}
$$

According to the third and fourth rules of Fig. 3.1, the matrix elements for the two Feynman graphs are:

$$
\begin{aligned}
i \mathcal{M}_{a} & =i \frac{g}{c_{W}}\left(T_{3}^{f}-s_{W}^{2} Q_{f}\right) \varepsilon_{\mu} x_{f}^{\dagger} \bar{\sigma}^{\mu} y_{\bar{f}}, \\
i \mathcal{M}_{b} & =-i g \frac{s_{W}^{2}}{c_{W}} Q_{f} \varepsilon_{\mu} y_{f} \sigma^{\mu} x_{\bar{f}}^{\dagger},
\end{aligned}
$$

where $x_{i} \equiv x\left(\overrightarrow{\boldsymbol{k}}_{i}, \lambda_{i}\right)$ and $y_{i} \equiv y\left(\overrightarrow{\boldsymbol{k}}_{\boldsymbol{i}}, \lambda_{i}\right)$, for $i=f, \bar{f}$, and $\varepsilon_{\mu} \equiv \varepsilon_{\mu}\left(p, \lambda_{Z}\right)$.

It is convenient to define:

$$
a_{f} \equiv T_{3}^{f}-Q_{f} s_{W}^{2}, \quad b_{f} \equiv-Q_{f} s_{W}^{2} .
$$

Then the squared matrix element is, using eqs. (2.1.31) and (2.1.32),

$$
|\mathcal{M}|^{2}=\frac{g^{2}}{c_{W}^{2}} \varepsilon_{\mu} \varepsilon_{\nu}^{*}\left(a_{f} x_{f}^{\dagger} \bar{\sigma}^{\mu} y_{\bar{f}}+b_{f} y_{f} \sigma^{\mu} x_{\bar{f}}^{\dagger}\right)\left(a_{f} y_{\bar{f}}^{\dagger} \bar{\sigma}^{\nu} x_{f}+b_{f} x_{\bar{f}} \sigma^{\nu} y_{f}^{\dagger}\right) .
$$

Summing over the antifermion helicity using eqs. (2.3.3)-(2.3.6) gives:

$$
\begin{array}{r}
\sum_{\lambda_{\bar{f}}}|\mathcal{M}|^{2}=\frac{g^{2}}{c_{W}^{2}} \varepsilon_{\mu} \varepsilon_{\nu}^{*}\left(a_{f}^{2} x_{f}^{\dagger} \bar{\sigma}^{\mu} k_{\bar{f}} \cdot \sigma \bar{\sigma}^{\nu} x_{f}+b_{f}^{2} y_{f} \sigma^{\mu} k_{\bar{f}} \cdot \bar{\sigma} \sigma^{\nu} y_{f}^{\dagger}\right. \\
\left.-m_{f} a_{f} b_{f} x_{f}^{\dagger} \bar{\sigma}^{\mu} \sigma^{\nu} y_{f}^{\dagger}-m_{f} a_{f} b_{f} y_{f} \sigma^{\mu} \bar{\sigma}^{\nu} x_{f}\right) .
\end{array}
$$

Next, we sum over the fermion helicity:

$$
\begin{gathered}
\sum_{\lambda_{f}, \lambda_{\bar{f}}}|\mathcal{M}|^{2}=\frac{g^{2}}{c_{W}^{2}} \varepsilon_{\mu} \varepsilon_{\nu}^{*}\left(a_{f}^{2} \operatorname{Tr}\left[\bar{\sigma}^{\mu} k_{\bar{f}} \cdot \sigma \bar{\sigma}^{\nu} k_{f} \cdot \sigma\right]+b_{f}^{2} \operatorname{Tr}\left[\sigma^{\mu} k_{\bar{f}} \cdot \bar{\sigma} \sigma^{\nu} k_{f} \cdot \bar{\sigma}\right]\right. \\
\left.-m_{f}^{2} a_{f} b_{f} \operatorname{Tr}\left[\bar{\sigma}^{\mu} \sigma^{\nu}\right]-m_{f}^{2} a_{f} b_{f} \operatorname{Tr}\left[\sigma^{\mu} \bar{\sigma}^{\nu}\right]\right) .
\end{gathered}
$$

Averaging over the $Z^{0}$ polarization using

$$
\frac{1}{3} \sum_{\lambda_{Z}} \varepsilon_{\mu} \varepsilon_{\nu}^{*}=\frac{1}{3}\left(g_{\mu \nu}+\frac{p_{\mu} p_{\nu}}{m_{Z}^{2}}\right),
$$

and applying eqs. (2.1.43)-(2.1.45), one gets:

$$
\begin{aligned}
\frac{1}{3} \sum_{\text {spins }}|\mathcal{M}|^{2} & =\frac{g^{2}}{3 c_{W}^{2}}\left[\left(a_{f}^{2}+b_{f}^{2}\right)\left(-2 k_{f} \cdot k_{\bar{f}}+4 k_{f} \cdot p k_{\bar{f}} \cdot p / m_{Z}^{2}\right)+12 a_{f} b_{f} m_{f}^{2}\right] \\
& =\frac{2 g^{2}}{3 c_{W}^{2}}\left[\left(a_{f}^{2}+b_{f}^{2}\right)\left(m_{Z}^{2}-m_{f}^{2}\right)+6 a_{f} b_{f} m_{f}^{2}\right]
\end{aligned}
$$


where we have used eqs. (5.2.1) and (5.2.2). After the standard phase space integration, we obtain the well-known result:

$$
\begin{aligned}
& \Gamma\left(Z^{0} \rightarrow f \bar{f}\right)=\frac{N_{c}^{f}}{16 \pi m_{Z}}\left(1-\frac{4 m_{f}^{2}}{m_{Z}^{2}}\right)^{1 / 2}\left(\frac{1}{3} \sum_{\text {spins }}|\mathcal{M}|^{2}\right) \\
& =\frac{N_{c}^{f} g^{2} m_{Z}}{24 \pi c_{W}^{2}}\left(1-\frac{4 m_{f}^{2}}{m_{Z}^{2}}\right)^{1 / 2}\left[\left(a_{f}^{2}+b_{f}^{2}\right)\left(1-\frac{m_{f}^{2}}{m_{Z}^{2}}\right)+6 a_{f} b_{f} \frac{m_{f}^{2}}{m_{Z}^{2}}\right] .
\end{aligned}
$$

Here we have also included a factor of $N_{c}^{f}$ (equal to 1 for leptons and 3 for quarks) for the sum over colors. Since the $Z^{0}$ is a color singlet, the color factor is simply equal to the dimension of the color representation of the final-state fermions.

\subsection{Bhabha scattering: $e^{-} e^{+} \rightarrow e^{-} e^{+}$}

In our next example, we consider the computation of Bhabha scattering in QED (that is, we consider photon exchange but neglect $Z^{0}$-exchange). We denote the initial state electron and positron momenta and helicities by $\left(p_{1}, \lambda_{1}\right)$ and $\left(p_{2}, \lambda_{2}\right)$ and the final state electron and positron momenta and helicities by $\left(p_{3}, \lambda_{3}\right)$ and $\left(p_{4}, \lambda_{4}\right)$, respectively. Neglecting the electron mass, we have in terms of the usual Mandelstam variables $s, t, u$ :

$$
\begin{aligned}
& p_{1} \cdot p_{2}=p_{3} \cdot p_{4} \equiv-\frac{1}{2} s, \\
& p_{1} \cdot p_{3}=p_{2} \cdot p_{4} \equiv \frac{1}{2} t, \\
& p_{1} \cdot p_{4}=p_{2} \cdot p_{3} \equiv \frac{1}{2} u,
\end{aligned}
$$

and $p_{i}^{2}=0$ for $i=1, \ldots, 4$. There are eight distinct Feynman diagrams. First, there are four $s$-channel diagrams, as shown in Fig. 5.3.1 with amplitudes that follow from the first and second Feynman rules of Fig. 3.1.

$$
i \mathcal{M}_{s}=\left(\frac{i g^{\mu \nu}}{s}\right)\left[\left(i e x_{1} \sigma_{\mu} y_{2}^{\dagger}\right)\left(-i e y_{3} \sigma_{\nu} x_{4}^{\dagger}\right)+\left(i e y_{1}^{\dagger} \bar{\sigma}_{\mu} x_{2}\right)\left(-i e y_{3} \sigma_{\nu} x_{4}^{\dagger}\right)\right.
$$
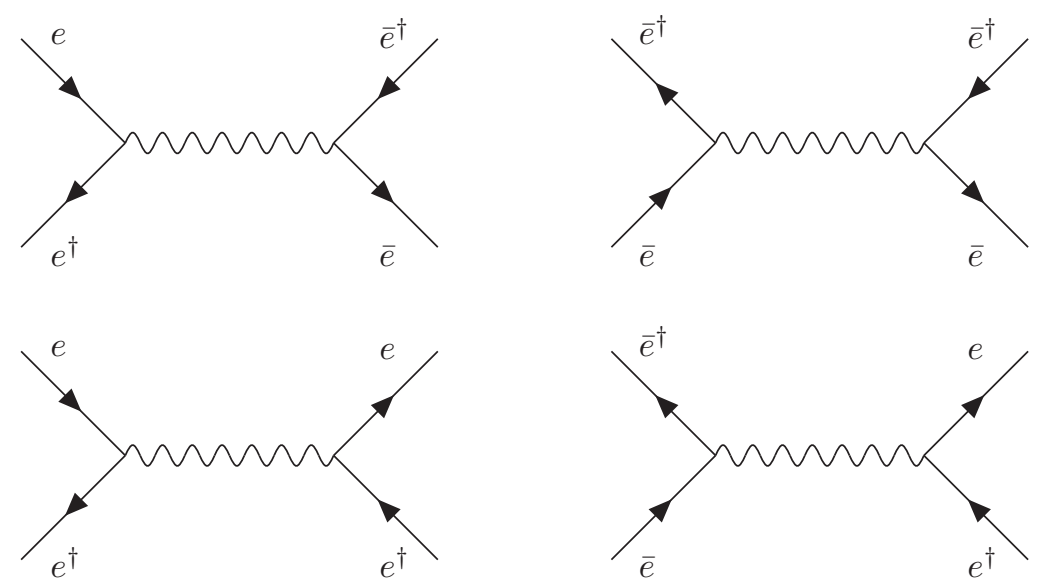

Fig. 5.3.1: Tree-level $s$-channel Feynman diagrams for $e^{+} e^{-} \rightarrow e^{+} e^{-}$. 

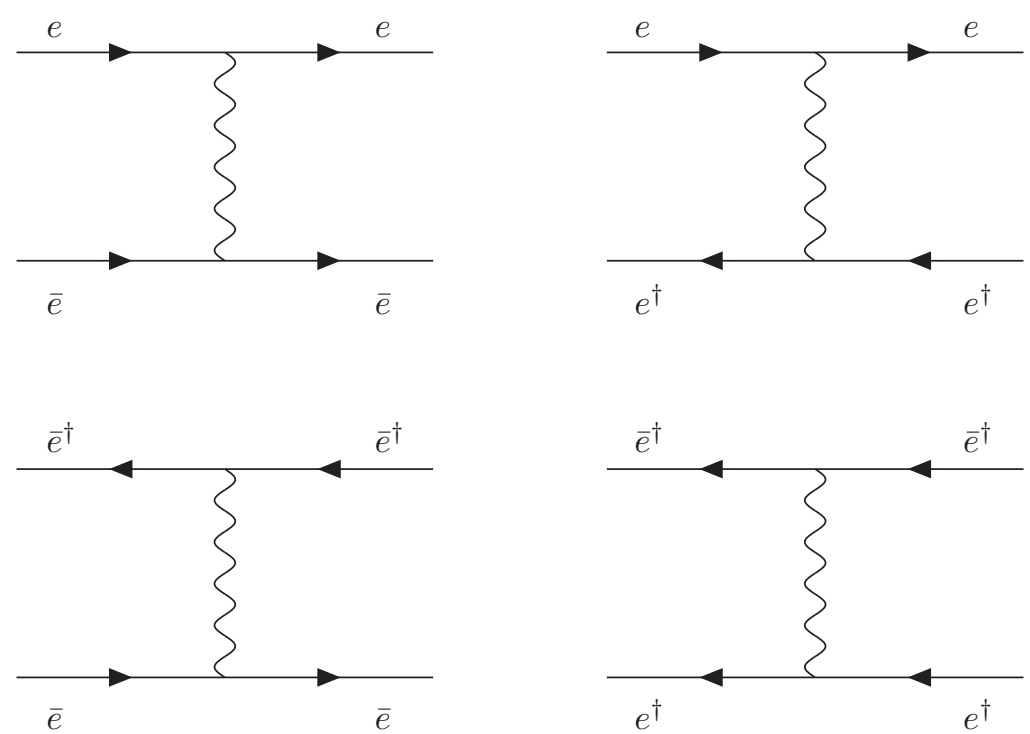

Fig. 5.3.2: Tree-level $t$-channel Feynman diagrams for $e^{-} e^{+} \rightarrow e^{-} e^{+}$, with the external lines labeled according to the 2-component field names. The momentum flow of the external particles is from left to right.

$$
\left.+\left(i e x_{1} \sigma_{\mu} y_{2}^{\dagger}\right)\left(-i e x_{3}^{\dagger} \bar{\sigma}_{\nu} y_{4}\right)+\left(i e y_{1}^{\dagger} \bar{\sigma}_{\mu} x_{2}\right)\left(-i e x_{3}^{\dagger} \bar{\sigma}_{\nu} y_{4}\right)\right]
$$

where $x_{i} \equiv x\left(\overrightarrow{\boldsymbol{p}}_{\boldsymbol{i}}, \lambda_{i}\right)$ and $y_{i} \equiv y\left(\overrightarrow{\boldsymbol{p}}_{\boldsymbol{i}}, \lambda_{i}\right)$, for $i=1,4$. The photon propagator in Feynman gauge is $-i g^{\mu \nu} /\left(p_{1}+p_{2}\right)^{2}=i g^{\mu \nu} / s$. Here, we have chosen to write the external fermion spinors in the order $1,2,3,4$. This dictates in each term the use of either the $\bar{\sigma}$ or $\sigma$ forms of the Feynman rules of Fig. 3.1. One can group the terms of eq. (5.3.4) together more compactly:

$$
i \mathcal{M}_{s}=e^{2}\left(\frac{i g^{\mu \nu}}{s}\right)\left(x_{1} \sigma_{\mu} y_{2}^{\dagger}+y_{1}^{\dagger} \bar{\sigma}_{\mu} x_{2}\right)\left(y_{3} \sigma_{\nu} x_{4}^{\dagger}+x_{3}^{\dagger} \bar{\sigma}_{\nu} y_{4}\right) .
$$

There are also four $t$-channel diagrams, as shown in Fig. 5.3.2. The corresponding amplitudes for these four diagrams can be written:

$$
i \mathcal{M}_{t}=(-1) e^{2}\left(\frac{i g^{\mu \nu}}{t}\right)\left(x_{1} \sigma_{\mu} x_{3}^{\dagger}+y_{1}^{\dagger} \bar{\sigma}_{\mu} y_{3}\right)\left(x_{2} \sigma_{\nu} x_{4}^{\dagger}+y_{2}^{\dagger} \bar{\sigma}_{\nu} y_{4}\right) .
$$

Here, the overall factor of $(-1)$ comes from Fermi-Dirac statistics, since the external fermion wave functions are written in an odd permutation $(1,3,2,4)$ of the original order $(1,2,3,4)$ established by the first term in eq. (5.3.4).

Fierzing each term using eqs. (2.1.55)-(2.1.57), and using eqs. (2.1.47) and (2.1.48), the total amplitude can be written as:

$$
\begin{aligned}
\mathcal{M}= & \mathcal{M}_{s}+\mathcal{M}_{t}=2 e^{2}\left[\frac{1}{s}\left(x_{1} y_{3}\right)\left(y_{2}^{\dagger} x_{4}^{\dagger}\right)+\frac{1}{s}\left(y_{1}^{\dagger} x_{3}^{\dagger}\right)\left(x_{2} y_{4}\right)\right. \\
& +\left(\frac{1}{s}+\frac{1}{t}\right)\left(y_{1}^{\dagger} x_{4}^{\dagger}\right)\left(x_{2} y_{3}\right)+\left(\frac{1}{s}+\frac{1}{t}\right)\left(x_{1} y_{4}\right)\left(y_{2}^{\dagger} x_{3}^{\dagger}\right) \\
& \left.-\frac{1}{t}\left(x_{1} x_{2}\right)\left(x_{3}^{\dagger} x_{4}^{\dagger}\right)-\frac{1}{t}\left(y_{1}^{\dagger} y_{2}^{\dagger}\right)\left(y_{3} y_{4}\right)\right] .
\end{aligned}
$$


Squaring this amplitude and summing over spins, all of the cross terms will vanish in the $m_{e} \rightarrow 0$ limit. This is because each cross term will have an $x$ or an $x^{\dagger}$ for some electron or positron combined with a $y$ or a $y^{\dagger}$ for the same particle, and the corresponding spin sum is proportional to $m_{e}$ [see eqs. (2.3.5) and (2.3.6)]. Hence, summing over final state spins and averaging over initial state spins, the end result contains only the sum of the squares of the six terms in eq. (5.3.7):

$$
\begin{aligned}
& \frac{1}{4} \sum_{\text {spins }}|\mathcal{M}|^{2}=e^{4} \sum_{\lambda_{1}, \lambda_{2}, \lambda_{3}, \lambda_{4}}\{ \\
& \frac{1}{s^{2}}\left[\left(x_{1} y_{3}\right)\left(y_{3}^{\dagger} x_{1}^{\dagger}\right)\left(y_{2}^{\dagger} x_{4}^{\dagger}\right)\left(x_{4} y_{2}\right)+\left(y_{1}^{\dagger} x_{3}^{\dagger}\right)\left(x_{3} y_{1}\right)\left(x_{2} y_{4}\right)\left(y_{4}^{\dagger} x_{2}^{\dagger}\right)\right] \\
& +\left(\frac{1}{s}+\frac{1}{t}\right)^{2}\left[\left(y_{1}^{\dagger} x_{4}^{\dagger}\right)\left(x_{4} y_{1}\right)\left(x_{2} y_{3}\right)\left(y_{3}^{\dagger} x_{2}^{\dagger}\right)+\left(x_{1} y_{4}\right)\left(y_{4}^{\dagger} x_{1}^{\dagger}\right)\left(y_{2}^{\dagger} x_{3}^{\dagger}\right)\left(x_{3} y_{2}\right)\right] \\
& \left.+\frac{1}{t^{2}}\left[\left(x_{1} x_{2}\right)\left(x_{2}^{\dagger} x_{1}^{\dagger}\right)\left(x_{3}^{\dagger} x_{4}^{\dagger}\right)\left(x_{4} x_{3}\right)+\left(y_{1}^{\dagger} y_{2}^{\dagger}\right)\left(y_{2} y_{1}\right)\left(y_{3} y_{4}\right)\left(y_{4}^{\dagger} y_{3}^{\dagger}\right)\right]\right\} .
\end{aligned}
$$

Here we have used eq. (2.1.30) to get the complex square of the fermion bilinears. Performing these spin sums using eqs. (2.3.3) and (2.3.4) and using the trace identities eq. (2.1.43):

$$
\begin{aligned}
\frac{1}{4} \sum_{\text {spins }}|\mathcal{M}|^{2} & =8 e^{4}\left[\frac{p_{2} \cdot p_{4} p_{1} \cdot p_{3}}{s^{2}}+\frac{p_{1} \cdot p_{2} p_{3} \cdot p_{4}}{t^{2}}+\left(\frac{1}{s}+\frac{1}{t}\right)^{2} p_{1} \cdot p_{4} p_{2} \cdot p_{3}\right] \\
& =2 e^{4}\left[\frac{t^{2}}{s^{2}}+\frac{s^{2}}{t^{2}}+\left(\frac{u}{s}+\frac{u}{t}\right)^{2}\right] .
\end{aligned}
$$

Thus, the differential cross-section for Bhabha scattering is given by:

$$
\frac{d \sigma}{d t}=\frac{1}{16 \pi s^{2}}\left(\frac{1}{4} \sum_{\text {spins }}|\mathcal{M}|^{2}\right)=\frac{2 \pi \alpha^{2}}{s^{2}}\left[\frac{t^{2}}{s^{2}}+\frac{s^{2}}{t^{2}}+\left(\frac{u}{s}+\frac{u}{t}\right)^{2}\right] \text {. }
$$

\subsection{Neutral MSSM Higgs boson decays $\phi^{0} \rightarrow f \bar{f}$, for $\phi^{0}=h^{0}, H^{0}, A^{0}$}

In this subsection, we consider the decays of the neutral Higgs scalar bosons $\phi^{0}=h^{0}$, $H^{0}$, and $A^{0}$ of the MSSM into Standard Model fermion-antifermion pairs. The relevant tree-level Feynman diagrams are shown in Fig. 5.4.1. The final state fermion is assigned four-momentum $p_{1}$ and polarization $\lambda_{1}$, and the antifermion is assigned four-momentum $p_{2}$ and polarization $\lambda_{2}$. We will first work out the case that $f$ is a charge $-1 / 3$ quark or a charged lepton, and later note the simple change needed for charge $+2 / 3$ quarks. The second and fifth Feynman rules of Fig. 4.3 tell us that the amplitudes are:

$$
\begin{aligned}
i \mathcal{M}_{a} & =-\frac{i}{\sqrt{2}} Y_{f} k_{d \phi^{0}}^{*} x_{1}^{\dagger} x_{2}^{\dagger}, \\
i \mathcal{M}_{b} & =-\frac{i}{\sqrt{2}} Y_{f} k_{d \phi^{0}} y_{1} y_{2} .
\end{aligned}
$$

Here $Y_{f}$ is the Yukawa coupling of the fermion, $k_{d \phi^{0}}$ is the Higgs mixing parameter from eq. (4.16), and the external wave functions are denoted $x_{1} \equiv x\left(\overrightarrow{\boldsymbol{p}}_{1}, \lambda_{1}\right), y_{1} \equiv y\left(\overrightarrow{\boldsymbol{p}}_{1}, \lambda_{1}\right)$ for 


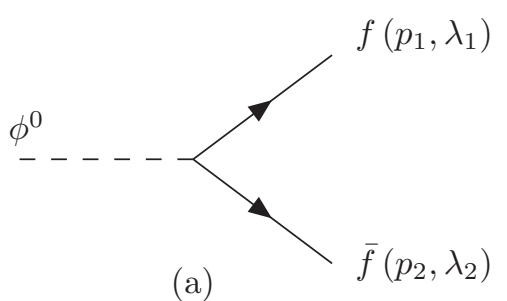

(a)

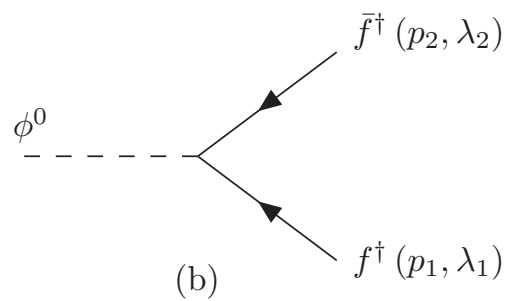

(b)

Fig. 5.4.1: The Feynman diagrams for the decays $\phi^{0} \rightarrow f \bar{f}$, where $\phi^{0}=h^{0}, H^{0}, A^{0}$ are the neutral Higgs scalar bosons of the MSSM, and $f$ is a Standard Model quark or lepton, and $\bar{f}$ is the corresponding antiparticle. The external fermions are labeled according to the 2-component field names.

the fermion and $x_{2} \equiv x\left(\overrightarrow{\boldsymbol{p}}_{2}, \lambda_{2}\right), y_{2} \equiv y\left(\overrightarrow{\boldsymbol{p}}_{2}, \lambda_{2}\right)$ for the antifermion. Squaring the total amplitude $i \mathcal{M}=i \mathcal{M}_{a}+i \mathcal{M}_{b}$ using eq. (2.1.30) results in:

$$
\begin{aligned}
|\mathcal{M}|^{2}= & \frac{1}{2}\left|Y_{f}\right|^{2}\left[\left|k_{d \phi^{0}}\right|^{2}\left(y_{1} y_{2} y_{2}^{\dagger} y_{1}^{\dagger}+x_{1}^{\dagger} x_{2}^{\dagger} x_{2} x_{1}\right)\right. \\
& \left.+\left(k_{d \phi^{0}}^{*}\right)^{2} x_{1}^{\dagger} x_{2}^{\dagger} y_{2}^{\dagger} y_{1}^{\dagger}+\left(k_{d \phi^{0}}\right)^{2} y_{1} y_{2} x_{2} x_{1}\right] .
\end{aligned}
$$

Summing over the final state antifermion spin using eqs. (2.3.3)-(2.3.6) gives:

$$
\begin{aligned}
\sum_{\lambda_{2}}|\mathcal{M}|^{2}= & \frac{1}{2}\left|Y_{f}\right|^{2}\left[-\left|k_{d \phi^{0}}\right|^{2}\left(y_{1} p_{2} \cdot \sigma y_{1}^{\dagger}+x_{1}^{\dagger} p_{2} \cdot \bar{\sigma} x_{1}\right)\right. \\
& \left.-\left(k_{d \phi^{0}}^{*}\right)^{2} m_{f} x_{1}^{\dagger} y_{1}^{\dagger}-\left(k_{d \phi^{0}}\right)^{2} m_{f} y_{1} x_{1}\right] .
\end{aligned}
$$

Summing over the fermion spins in the same way yields:

$$
\begin{aligned}
\sum_{\lambda_{1}, \lambda_{2}}|\mathcal{M}|^{2}= & \frac{1}{2}\left|Y_{f}\right|^{2}\left\{\left|k_{d \phi^{0}}\right|^{2}\left(\operatorname{Tr}\left[p_{2} \cdot \sigma p_{1} \cdot \bar{\sigma}\right]+\operatorname{Tr}\left[p_{2} \cdot \bar{\sigma} p_{1} \cdot \sigma\right]\right)\right. \\
& \left.-2\left(k_{d \phi^{0}}^{*}\right)^{2} m_{f}^{2}-2\left(k_{d \phi^{0}}\right)^{2} m_{f}^{2}\right\} \\
= & \left|Y_{f}\right|^{2}\left\{-2\left|k_{d \phi^{0}}\right|^{2} p_{1} \cdot p_{2}-2 \operatorname{Re}\left[\left(k_{d \phi^{0}}\right)^{2}\right] m_{f}^{2}\right\} \\
= & \left|Y_{f}\right|^{2}\left\{\left|k_{d \phi^{0}}\right|^{2}\left(m_{\phi^{0}}^{2}-2 m_{f}^{2}\right)-2 \operatorname{Re}\left[\left(k_{d \phi^{0}}\right)^{2}\right] m_{f}^{2}\right\},
\end{aligned}
$$

where we have used the trace identity eq. (2.1.43) to obtain the second equality. The corresponding expression for charge $+2 / 3$ quarks can be obtained by simply replacing $k_{d \phi^{0}}$ with $k_{u \phi^{0}}$. The total decay rates now follow from integration over phase space

$$
\Gamma\left(\phi^{0} \rightarrow f \bar{f}\right)=\frac{N_{c}^{f}}{16 \pi m_{\phi^{0}}}\left(1-4 m_{f}^{2} / m_{\phi^{0}}^{2}\right)^{1 / 2} \sum_{\lambda_{1}, \lambda_{2}}|\mathcal{M}|^{2} .
$$

The factor of $N_{c}^{f}=3$ for quarks and 1 for leptons comes from the sum over colors.

Results for special cases are obtained by putting in the relevant values for the couplings and the mixing parameters from eqs. (4.15) and (4.16). In particular, for the CP-even Higgs bosons $h^{0}$ and $H^{0}, k_{d \phi^{0}}$ and $k_{u \phi^{0}}$ are real, so one obtains:

$$
\begin{aligned}
\Gamma\left(h^{0} \rightarrow b \bar{b}\right) & =\frac{3}{16 \pi} Y_{b}^{2} \sin ^{2} \alpha m_{h^{0}}\left(1-4 m_{b}^{2} / m_{h^{0}}^{2}\right)^{3 / 2}, \\
\Gamma\left(h^{0} \rightarrow c \bar{c}\right) & =\frac{3}{16 \pi} Y_{c}^{2} \cos ^{2} \alpha m_{h^{0}}\left(1-4 m_{c}^{2} / m_{h^{0}}^{2}\right)^{3 / 2},
\end{aligned}
$$




$$
\begin{aligned}
\Gamma\left(h^{0} \rightarrow \tau^{+} \tau^{-}\right) & =\frac{1}{16 \pi} Y_{\tau}^{2} \sin ^{2} \alpha m_{h^{0}}\left(1-4 m_{\tau}^{2} / m_{h^{0}}^{2}\right)^{3 / 2}, \\
\Gamma\left(H^{0} \rightarrow t \bar{t}\right) & =\frac{3}{16 \pi} Y_{t}^{2} \sin ^{2} \alpha m_{H^{0}}\left(1-4 m_{t}^{2} / m_{H^{0}}^{2}\right)^{3 / 2}, \\
\Gamma\left(H^{0} \rightarrow b \bar{b}\right) & =\frac{3}{16 \pi} Y_{b}^{2} \cos ^{2} \alpha m_{H^{0}}\left(1-4 m_{b}^{2} / m_{H^{0}}^{2}\right)^{3 / 2},
\end{aligned}
$$

etc., which check with the expressions in Appendix $\mathrm{C}$ of ref. [20]. For the CP-odd Higgs boson $A^{0}$, the mixing parameters $k_{u A^{0}}=i \cos \beta_{0}$ and $k_{d A^{0}}=i \sin \beta_{0}$ are purely imaginary, so

$$
\begin{aligned}
\Gamma\left(A^{0} \rightarrow t \bar{t}\right) & =\frac{3}{16 \pi} Y_{t}^{2} \cos ^{2} \beta_{0} m_{A^{0}}\left(1-4 m_{t}^{2} / m_{A^{0}}^{2}\right)^{1 / 2}, \\
\Gamma\left(A^{0} \rightarrow b \bar{b}\right) & =\frac{3}{16 \pi} Y_{b}^{2} \sin ^{2} \beta_{0} m_{A^{0}}\left(1-4 m_{b}^{2} / m_{A^{0}}^{2}\right)^{1 / 2}, \\
\Gamma\left(A^{0} \rightarrow \tau^{+} \tau^{-}\right) & =\frac{1}{16 \pi} Y_{\tau}^{2} \sin ^{2} \beta_{0} m_{A^{0}}\left(1-4 m_{\tau}^{2} / m_{A^{0}}^{2}\right)^{1 / 2} .
\end{aligned}
$$

The differing kinematic factors for the CP-odd Higgs decays came about because of the different relative sign between the two Feynman diagrams. For example, in the case of $h^{0} \rightarrow b \bar{b}$, the matrix element is

$$
i \mathcal{M}=\frac{i}{\sqrt{2}} Y_{b} \sin \alpha\left(y_{1} y_{2}+x_{1}^{\dagger} x_{2}^{\dagger}\right)
$$

while for $A^{0} \rightarrow b \bar{b}$, it is

$$
i \mathcal{M}=\frac{1}{\sqrt{2}} Y_{b} \sin \beta_{0}\left(y_{1} y_{2}-x_{1}^{\dagger} x_{2}^{\dagger}\right) .
$$

The differing relative sign between $y_{1} y_{2}$ and $x_{1}^{\dagger} x_{2}^{\dagger}$ follows from the imaginary pseudoscalar Lagrangian coupling, which is complex conjugated in the second diagram.

\subsection{Neutralino decays $\widetilde{N}_{i} \rightarrow \phi^{0} \widetilde{N}_{j}$, for $\phi^{0}=h^{0}, H^{0}, A^{0}$}

Next we consider the decay of a neutralino to a lighter neutralino and neutral Higgs boson $\phi^{0}=h^{0}, H^{0}$, or $A^{0}$. The two tree-level Feynman graphs are shown in Fig. 5.5.1, where we have also labeled the momenta and helicities. We denote the masses for the neutralinos and the Higgs boson as $m_{\widetilde{N}_{i}}, m_{\widetilde{N}_{j}}$, and $m_{\phi^{0}}$. Using the first Feynman rule of Fig. 4.5, the amplitudes are respectively given by

$$
i \mathcal{M}_{1}=-i Y x_{i} y_{j}, \quad i \mathcal{M}_{2}=-i Y^{*} y_{i}^{\dagger} x_{j}^{\dagger},
$$
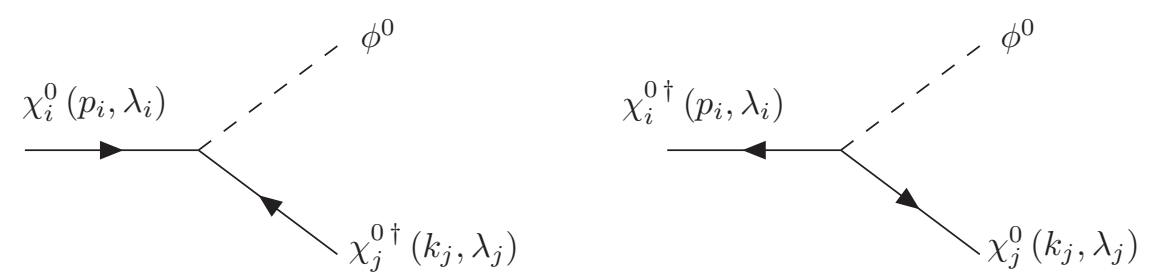

Fig. 5.5.1: The Feynman diagrams for $\widetilde{N}_{i} \rightarrow \widetilde{N}_{j} \phi^{0}$ in the MSSM. 
where the coupling $Y \equiv Y^{\phi^{0}} \chi_{i}^{0} \chi_{j}^{0}$ is defined in eq. (4.17), and the external wave functions are $x_{i} \equiv x\left(\overrightarrow{\boldsymbol{p}}_{i}, \lambda_{i}\right), y_{i}^{\dagger} \equiv y^{\dagger}\left(\overrightarrow{\boldsymbol{p}}_{i}, \lambda_{i}\right), y_{j} \equiv y\left(\overrightarrow{\boldsymbol{k}}_{j}, \lambda_{j}\right)$, and $x_{j}^{\dagger} \equiv x^{\dagger}\left(\overrightarrow{\boldsymbol{k}}_{j}, \lambda_{j}\right)$.

Taking the square of the total matrix element using eq. (2.1.30) gives:

$$
|\mathcal{M}|^{2}=|Y|^{2}\left(x_{i} y_{j} y_{j}^{\dagger} x_{i}^{\dagger}+y_{i}^{\dagger} x_{j}^{\dagger} x_{j} y_{i}\right)+Y^{2} x_{i} y_{j} x_{j} y_{i}+Y^{* 2} y_{i}^{\dagger} x_{j}^{\dagger} y_{j}^{\dagger} x_{i}^{\dagger} .
$$

Summing over the final state neutralino spin using eqs. (2.3.3)-(2.3.6) yields

$$
\begin{aligned}
\sum_{\lambda_{j}}|\mathcal{M}|^{2}= & -|Y|^{2}\left(x_{i} k_{j} \cdot \sigma x_{i}^{\dagger}+y_{i}^{\dagger} k_{j} \cdot \bar{\sigma} y_{i}\right) \\
& -Y^{2} m_{\widetilde{N}_{j}} x_{i} y_{i}-Y^{* 2} m_{\widetilde{N}_{j}} y_{i}^{\dagger} x_{i}^{\dagger} .
\end{aligned}
$$

Averaging over the initial state neutralino spins in the same way gives

$$
\begin{aligned}
\frac{1}{2} \sum_{\lambda_{i}, \lambda_{j}}|\mathcal{M}|^{2} & =\frac{1}{2}|Y|^{2}\left(\operatorname{Tr}\left[k_{j} \cdot \sigma p_{i} \cdot \bar{\sigma}\right]+\operatorname{Tr}\left[k_{j} \cdot \bar{\sigma} p_{i} \cdot \sigma\right]\right)+\operatorname{Re}\left[Y^{2}\right] m_{\widetilde{N}_{i}} m_{\widetilde{N}_{j}} \operatorname{Tr}[1] \\
& =-2|Y|^{2} p_{i} \cdot k_{j}+2 \operatorname{Re}\left[Y^{2}\right] m_{\widetilde{N}_{i}} m_{\widetilde{N}_{j}} \\
& =|Y|^{2}\left(m_{\widetilde{N}_{i}}^{2}+m_{\widetilde{N}_{j}}^{2}-m_{\phi^{0}}^{2}\right)+2 \operatorname{Re}\left[Y^{2}\right] m_{\widetilde{N}_{i}} m_{\widetilde{N}_{j}}
\end{aligned}
$$

where we have used eq. (2.1.43) to obtain the second equality. The total decay rate is therefore

$$
\begin{aligned}
\Gamma\left(\widetilde{N}_{i} \rightarrow \phi^{0} \widetilde{N}_{j}\right)= & \frac{1}{16 \pi m_{\widetilde{N}_{i}}^{3}} \lambda^{1 / 2}\left(m_{\widetilde{N}_{i}}^{2}, m_{\phi^{0}}^{2}, m_{\widetilde{N}_{j}}^{2}\right)\left(\frac{1}{2} \sum_{\lambda_{i}, \lambda_{j}}|\mathcal{M}|^{2}\right) \\
= & \frac{m_{\widetilde{N}_{i}}}{16 \pi} \lambda^{1 / 2}\left(1, r_{\phi}, r_{j}\right)\left\{\left|Y^{\phi^{0} \chi_{i}^{0} \chi_{j}^{0}}\right|^{2}\left(1+r_{j}-r_{\phi}\right)\right. \\
& \left.+2 \operatorname{Re}\left[\left(Y^{\phi^{0} \chi_{i}^{0} \chi_{j}^{0}}\right)^{2}\right] \sqrt{r_{j}}\right\},
\end{aligned}
$$

where the triangle function $\lambda^{1 / 2}$ is defined in eq. (5.1.13), $r_{j} \equiv m_{\widetilde{N}_{j}}^{2} / m_{\widetilde{N}_{i}}^{2}$ and $r_{\phi} \equiv$ $m_{\phi^{0}}^{2} / m_{\widetilde{N}_{i}}^{2}$. The results for $\phi^{0}=h^{0}, H^{0}, A^{0}$ can now be obtained by using eqs. (4.15) and (4.16) in eq. (4.17). In comparing eq. (5.5.5) with the original calculation in ref. [21], it is helpful to employ eqs. (4.51) and (4.53) of ref. 22. The results agree.

\section{6. $\widetilde{N}_{i} \rightarrow Z^{0} \widetilde{N}_{j}$}

For this two-body decay there are two tree-level Feynman diagrams, shown in Fig. 5.6.1 with the definitions of the helicities and the momenta. The two amplitudes are given byd

$$
\begin{aligned}
i \mathcal{M}_{1} & =i \frac{g}{c_{W}} \mathcal{O}_{j i}^{\prime L} x_{i} \sigma^{\mu} x_{j}^{\dagger} \varepsilon_{\mu}^{*}, \\
i \mathcal{M}_{2} & =-i \frac{g}{c_{W}} \mathcal{O}_{i j}^{\prime \prime L} y_{i}^{\dagger} \bar{\sigma}^{\mu} y_{j} \varepsilon_{\mu}^{*},
\end{aligned}
$$

where we have used the fifth Feynman rule of Fig. 4.2 in its $-\sigma$ and $\bar{\sigma}$ forms, and the external wave functions are $x_{i}=x\left(\overrightarrow{\boldsymbol{p}}_{i}, \lambda_{i}\right), y_{i}^{\dagger}=y^{\dagger}\left(\overrightarrow{\boldsymbol{p}}_{i}, \lambda_{i}\right), x_{j}^{\dagger}=x^{\dagger}\left(\overrightarrow{\boldsymbol{k}}_{j}, \lambda_{j}\right), y_{j}=y\left(\overrightarrow{\boldsymbol{k}}_{j}, \lambda_{j}\right)$,

\footnotetext{
${ }^{\mathrm{d}}$ When comparing with the 4-component Feynman rule in ref. [1] note that $\mathcal{O}_{i j}^{\prime \prime L}=-\mathcal{O}_{i j}^{\prime \prime R *}$ [cf. eq. 4.9] above].
} 

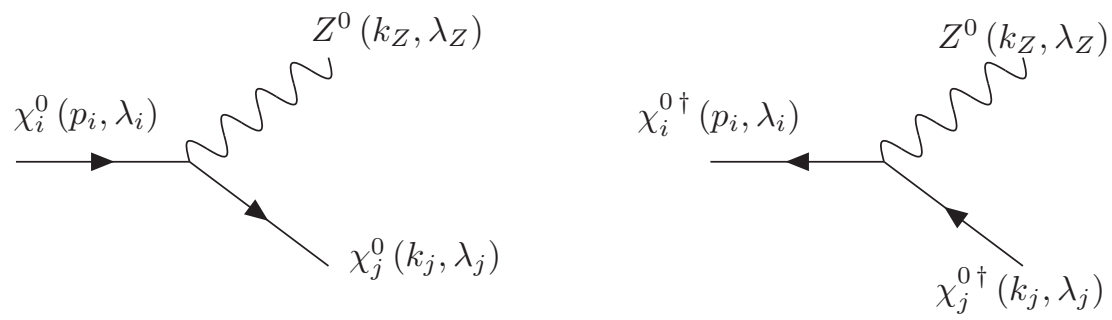

Fig. 5.6.1: The Feynman diagrams for $\widetilde{N}_{i} \rightarrow \widetilde{N}_{j} Z^{0}$ in the MSSM.

and $\varepsilon_{\mu}^{*}=\varepsilon_{\mu}\left(\overrightarrow{\boldsymbol{k}}_{Z}, \lambda_{Z}\right)^{*}$. Noting that $\mathcal{O}_{j i}^{\prime \prime L}=\mathcal{O}_{i j}^{\prime \prime L *}[$ see eq. (4.9)], and applying eqs. (2.1.31) and (2.1.32), we find that the squared matrix element is:

$$
\begin{aligned}
|\mathcal{M}|^{2}= & \frac{g^{2}}{c_{W}^{2}} \varepsilon_{\mu}^{*} \varepsilon_{\nu}\left[\left|\mathcal{O}_{i j}^{\prime \prime L}\right|^{2}\left(x_{i} \sigma^{\mu} x_{j}^{\dagger} x_{j} \sigma^{\nu} x_{i}^{\dagger}+y_{i}^{\dagger} \bar{\sigma}^{\mu} y_{j} y_{j}^{\dagger} \bar{\sigma}^{\nu} y_{i}\right)\right. \\
& \left.-\left(\mathcal{O}_{i j}^{\prime \prime L}\right)^{2} y_{i}^{\dagger} \bar{\sigma}^{\mu} y_{j} x_{j} \sigma^{\nu} x_{i}^{\dagger}-\left(\mathcal{O}_{i j}^{\prime \prime L *}\right)^{2} x_{i} \sigma^{\mu} x_{j}^{\dagger} y_{j}^{\dagger} \bar{\sigma}^{\nu} y_{i}\right] .
\end{aligned}
$$

Summing over the final state neutralino spin using eqs. (2.3.3)-(2.3.6) yields:

$$
\begin{aligned}
\sum_{\lambda_{j}}|\mathcal{M}|^{2}= & \frac{g^{2}}{c_{W}^{2}} \varepsilon_{\mu}^{*} \varepsilon_{\nu}\left[-\left|\mathcal{O}_{i j}^{\prime \prime L}\right|^{2}\left(x_{i} \sigma^{\mu} k_{j} \cdot \bar{\sigma} \sigma^{\nu} x_{i}^{\dagger}+y_{i}^{\dagger} \bar{\sigma}^{\mu} k_{j} \cdot \sigma \bar{\sigma}^{\nu} y_{i}\right)\right. \\
& \left.+\left(\mathcal{O}_{i j}^{\prime \prime L}\right)^{2} m_{\widetilde{N}_{j}} y_{i}^{\dagger} \bar{\sigma}^{\mu} \sigma^{\nu} x_{i}^{\dagger}+\left(\mathcal{O}_{i j}^{\prime \prime L *}\right)^{2} m_{\widetilde{N}_{j}} x_{i} \sigma^{\mu} \bar{\sigma}^{\nu} y_{i}\right] .
\end{aligned}
$$

Averaging over the initial state neutralino spin in the same way gives

$$
\begin{aligned}
\frac{1}{2} \sum_{\lambda_{i}, \lambda_{j}}|\mathcal{M}|^{2}= & \frac{g^{2}}{2 c_{W}^{2}} \varepsilon_{\mu}^{*} \varepsilon_{\nu}\left[\left|\mathcal{O}_{i j}^{\prime \prime L}\right|^{2}\left(\operatorname{Tr}\left[\sigma^{\mu} k_{j} \cdot \bar{\sigma} \sigma^{\nu} p_{i} \cdot \bar{\sigma}\right]+\operatorname{Tr}\left[\bar{\sigma}^{\mu} k_{j} \cdot \sigma \bar{\sigma}^{\nu} p_{i} \cdot \sigma\right]\right)\right. \\
& \left.-\left(\mathcal{O}_{i j}^{\prime \prime L}\right)^{2} m_{\widetilde{N}_{i}} m_{\widetilde{N}_{j}} \operatorname{Tr}\left[\bar{\sigma}^{\mu} \sigma^{\nu}\right]-\left(\mathcal{O}_{i j}^{\prime \prime L *}\right)^{2} m_{\widetilde{N}_{i}} m_{\widetilde{N}_{j}} \operatorname{Tr}\left[\sigma^{\mu} \bar{\sigma}^{\nu}\right]\right] \\
= & \frac{2 g^{2}}{c_{W}^{2}} \varepsilon_{\mu}^{*} \varepsilon_{\nu}\left\{\left|\mathcal{O}_{i j}^{\prime \prime L}\right|^{2}\left(k_{j}^{\mu} p_{i}^{\nu}+p_{i}^{\mu} k_{j}^{\nu}-p_{i} \cdot k_{j} g^{\mu \nu}\right)\right. \\
& \left.+\operatorname{Re}\left[\left(\mathcal{O}_{i j}^{\prime \prime L}\right)^{2}\right] m_{\widetilde{N}_{i}} m_{\widetilde{N}_{j}} g^{\mu \nu}\right\}
\end{aligned}
$$

where in the last equality we have applied eqs. (2.1.43)-(2.1.45). Using

$$
\sum_{\lambda_{Z}} \varepsilon^{\mu *} \varepsilon^{\nu}=g^{\mu \nu}+k_{Z}^{\mu} k_{Z}^{\nu} / m_{Z}^{2}
$$

we obtain

$$
\begin{aligned}
\frac{1}{2} \sum_{\lambda_{i}, \lambda_{j}, \lambda_{Z}}|\mathcal{M}|^{2}= & \frac{2 g^{2}}{c_{W}^{2}}\left\{\left|\mathcal{O}_{i j}^{\prime \prime L}\right|^{2}\left(-p_{i} \cdot k_{j}+2 p_{i} \cdot k_{Z} k_{j} \cdot k_{Z} / m_{Z}^{2}\right)\right. \\
& \left.+3 m_{\widetilde{N}_{i}} m_{\widetilde{N}_{j}} \operatorname{Re}\left[\left(\mathcal{O}_{i j}^{\prime \prime L}\right)^{2}\right]\right\} .
\end{aligned}
$$


Using $2 k_{j} \cdot k_{Z}=-m_{\widetilde{N}_{i}}^{2}+m_{\widetilde{N}_{j}}^{2}+m_{Z}^{2}, 2 p_{i} \cdot k_{j}=-m_{\widetilde{N}_{i}}^{2}-m_{\widetilde{N}_{j}}^{2}+m_{Z}^{2}$, and $2 p_{i} \cdot k_{Z}=-m_{\widetilde{N}_{i}}^{2}+$ $m_{\widetilde{N}_{j}}^{2}-m_{Z}^{2}$, we obtain the total decay width:

$$
\begin{aligned}
\Gamma\left(\widetilde{N}_{i}\right. & \left.\rightarrow Z^{0} \widetilde{N}_{j}\right)=\frac{1}{16 \pi m_{\widetilde{N}_{i}}^{3}} \lambda^{1 / 2}\left(m_{\widetilde{N}_{i}}^{2}, m_{Z}^{2}, m_{\widetilde{N}_{j}}^{2}\right)\left(\frac{1}{2} \sum_{\lambda_{i}, \lambda_{j}, \lambda_{Z}}|\mathcal{M}|^{2}\right) \\
= & \frac{g^{2} m_{\widetilde{N}_{i}}}{16 \pi c_{W}^{2}} \lambda^{1 / 2}\left(1, r_{Z}, r_{j}\right)\left[\left|\mathcal{O}_{i j}^{\prime \prime L}\right|^{2}\left(1+r_{j}-2 r_{Z}+\left(1-r_{j}\right)^{2} / r_{Z}\right)\right. \\
& \left.+6 \operatorname{Re}\left[\left(\mathcal{O}_{i j}^{\prime \prime L}\right)^{2}\right] \sqrt{r_{j}}\right],
\end{aligned}
$$

where

$$
r_{j} \equiv m_{\widetilde{N}_{j}}^{2} / m_{\widetilde{N}_{i}}^{2}, \quad r_{Z} \equiv m_{Z}^{2} / m_{\widetilde{N}_{i}}^{2},
$$

and the triangle function $\lambda^{1 / 2}$ is defined in eq. (5.1.13). The result obtained in eq. (5.6.9) agrees with the original calculation in ref. [21].

\section{7. $e^{-} e^{+} \rightarrow \widetilde{N}_{i} \widetilde{N}_{j}$}

Next we consider the pair production of neutralinos via $e^{-} e^{+}$annihilation. There are four Feynman graphs for $s$-channel $Z^{0}$ exchange, shown in Fig. 5.7.1, and four for $t / u$ channel selectron exchange, shown in Fig. 5.7.2. The momenta and polarizations are as labeled in the graphs. We denote the neutralino masses as $m_{\widetilde{N}_{i}}, m_{\widetilde{N}_{j}}$ and the selectron masses as $m_{\widetilde{e}_{L}}$ and $m_{\widetilde{e}_{R}}$. The electron mass will again be neglected. The kinematic variables are then given by

$$
\begin{aligned}
& s=-2 p_{1} \cdot p_{2}=m_{\widetilde{N}_{i}}^{2}+m_{\widetilde{N}_{j}}^{2}-2 k_{i} \cdot k_{j}, \\
& t=m_{\widetilde{N}_{i}}^{2}+2 p_{1} \cdot k_{i}=m_{\widetilde{N}_{j}}^{2}+2 p_{2} \cdot k_{j}, \\
& u=m_{\widetilde{N}_{i}}^{2}+2 p_{2} \cdot k_{i}=m_{\widetilde{N}_{j}}^{2}+2 p_{1} \cdot k_{j} .
\end{aligned}
$$

By applying the third and fourth Feynman rules of Figure 3.1 and the fifth of Figure 4.2. we obtain for the sum of the $s$-channel diagrams in Fig. 5.7.1.

$$
\begin{aligned}
i \mathcal{M}_{Z}=\frac{i g^{\mu \nu}}{D_{Z}} & {\left[-\frac{i g\left(s_{W}^{2}-\frac{1}{2}\right)}{c_{W}} x_{1} \sigma_{\mu} y_{2}^{\dagger}-\frac{i g s_{W}^{2}}{c_{W}} y_{1}^{\dagger} \bar{\sigma}_{\mu} x_{2}\right] } \\
& {\left[-\frac{i g}{c_{W}} O_{i j}^{\prime \prime L} x_{i}^{\dagger} \bar{\sigma}_{\nu} y_{j}+\frac{i g}{c_{W}} O_{j i}^{\prime \prime L} y_{i} \sigma_{\nu} x_{j}^{\dagger}\right], }
\end{aligned}
$$

where $O_{i j}^{\prime \prime}$ is given in eq. (4.9), and $D_{Z} \equiv s-m_{Z}^{2}+i \Gamma_{Z} m_{Z}$. The fermion spinors are denoted by $x_{1} \equiv x\left(\overrightarrow{\boldsymbol{p}}_{1}, \lambda_{1}\right), y_{2}^{\dagger} \equiv y^{\dagger}\left(\overrightarrow{\boldsymbol{p}}_{2}, \lambda_{2}\right), x_{i}^{\dagger} \equiv x^{\dagger}\left(\overrightarrow{\boldsymbol{k}}_{i}, \lambda_{i}\right), y_{j} \equiv y\left(\overrightarrow{\boldsymbol{k}}_{j}, \lambda_{j}\right)$, etc. The matrix elements of the four diagrams have been combined by factorizing with respect to the common boson propagator. For the four $t / u$-channel diagrams, we obtain, by applying 

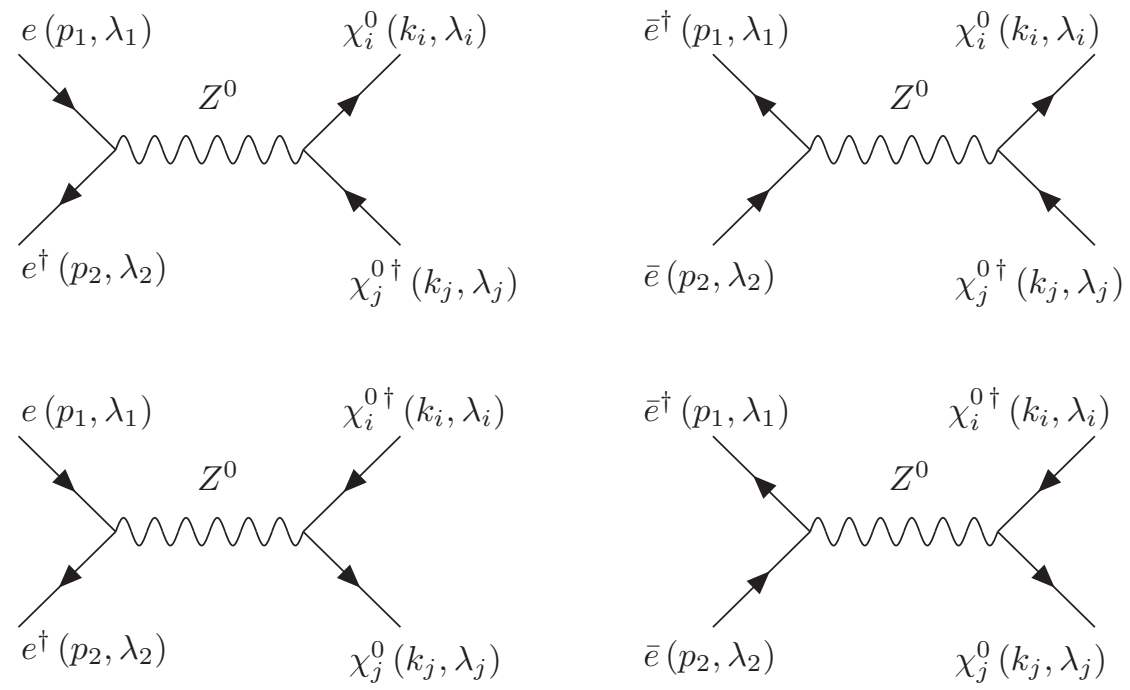

Fig. 5.7.1: The four Feynman diagrams for $e^{-} e^{+} \rightarrow \widetilde{N}_{i} \widetilde{N}_{j}$ via $s$-channel $Z^{0}$ exchange.
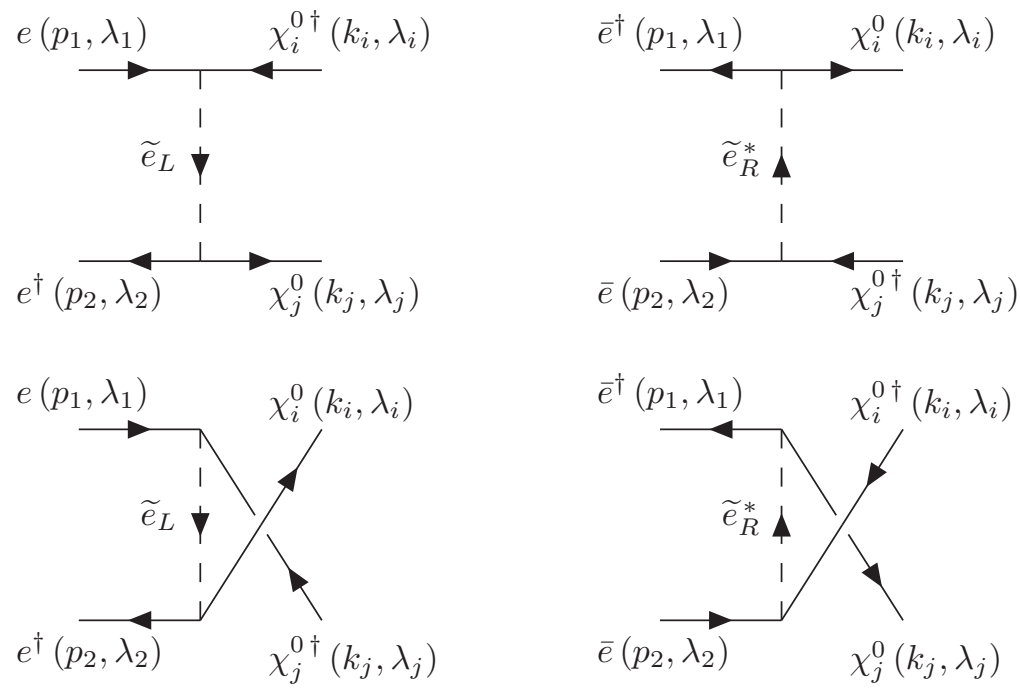

Fig. 5.7.2: The four Feynman diagrams for $e^{-} e^{+} \rightarrow \widetilde{N}_{i} \widetilde{N}_{j}$ via $t / u$-channel selectron exchange.

the first two rules of Fig. 4.7,

$$
\begin{aligned}
i \mathcal{M}_{\widetilde{e}_{L}}^{(t)}= & (-1)\left[\frac{i}{t-m_{\widetilde{e}_{L}}^{2}}\right]\left[\frac{i g}{\sqrt{2}}\left(N_{i 2}^{*}+\frac{s_{W}}{c_{W}} N_{i 1}^{*}\right)\right] \\
& {\left[\frac{i g}{\sqrt{2}}\left(N_{j 2}+\frac{s_{W}}{c_{W}} N_{j 1}\right)\right] x_{1} y_{i} y_{2}^{\dagger} x_{j}^{\dagger}, } \\
i \mathcal{M}_{\widetilde{e}_{L}}^{(u)}= & {\left[\frac{i}{u-m_{\widetilde{e}_{L}}^{2}}\right]\left[\frac{i g}{\sqrt{2}}\left(N_{j 2}^{*}+\frac{s_{W}}{c_{W}} N_{j 1}^{*}\right)\right] }
\end{aligned}
$$




$$
\begin{gathered}
{\left[\frac{i g}{\sqrt{2}}\left(N_{i 2}+\frac{s_{W}}{c_{W}} N_{i 1}\right)\right] x_{1} y_{j} y_{2}^{\dagger} x_{i}^{\dagger},} \\
i \mathcal{M}_{\widetilde{e}_{R}}^{(t)}=(-1) \frac{i}{t-m_{\widetilde{e}_{R}}^{2}}\left(-i \sqrt{2} g \frac{s_{W}}{c_{W}} N_{i 1}\right)\left(-i \sqrt{2} g \frac{s_{W}}{c_{W}} N_{j 1}^{*}\right) y_{1}^{\dagger} x_{i}^{\dagger} x_{2} y_{j}, \\
i \mathcal{M}_{\widetilde{e}_{R}}^{(u)}=\frac{i}{u-m_{\widetilde{e}_{R}}^{2}}\left(-i \sqrt{2} g \frac{s_{W}}{c_{W}} N_{j 1}\right)\left(-i \sqrt{2} g \frac{s_{W}}{c_{W}} N_{i 1}^{*}\right) y_{1}^{\dagger} x_{j}^{\dagger} x_{2} y_{i} .
\end{gathered}
$$

The first factors of $(-1)$ in each of eqs. (5.7.5) and (5.7.7) are present because the order of the spinors in each case is an odd permutation of the ordering $(1,2, i, j)$ established by the $s$-channel contribution. The other contributions have spinors in an even permutation of that ordering.

The $s$-channel diagram contribution of eq. (5.7.4) can be profitably rearranged using the Fierz identities of eqs. (2.1.55) and (2.1.56). Then, combining the result with the $t / u$-channel and $s$-channel contributions, we have for the total:

$$
\mathcal{M}=c_{1} x_{1} y_{j} y_{2}^{\dagger} x_{i}^{\dagger}+c_{2} x_{1} y_{i} y_{2}^{\dagger} x_{j}^{\dagger}+c_{3} y_{1}^{\dagger} x_{i}^{\dagger} x_{2} y_{j}+c_{4} y_{1}^{\dagger} x_{j}^{\dagger} x_{2} y_{i},
$$

where

$$
\begin{aligned}
c_{1}= & \frac{g^{2}}{c_{W}^{2}}\left[\left(1-2 s_{W}^{2}\right) O_{i j}^{\prime \prime L} / D_{Z}\right. \\
& \left.-\frac{1}{2}\left(c_{W} N_{i 2}+s_{W} N_{i 1}\right)\left(c_{W} N_{j 2}^{*}+s_{W} N_{j 1}^{*}\right) /\left(u-m_{\widetilde{e}_{L}}^{2}\right)\right], \\
c_{2}= & \frac{g^{2}}{c_{W}^{2}}\left[\left(2 s_{W}^{2}-1\right) O_{j i}^{\prime \prime L} / D_{Z}\right. \\
& \left.+\frac{1}{2}\left(c_{W} N_{i 2}^{*}+s_{W} N_{i 1}^{*}\right)\left(c_{W} N_{j 2}+s_{W} N_{j 1}\right) /\left(t-m_{\widetilde{e}_{L}}^{2}\right)\right], \\
c_{3}= & \frac{2 g^{2} s_{W}^{2}}{c_{W}^{2}}\left[-O_{i j}^{\prime \prime L} / D_{Z}+N_{i 1} N_{j 1}^{*} /\left(t-m_{\widetilde{e}_{R}}^{2}\right)\right], \\
c_{4}= & \frac{2 g^{2} s_{W}^{2}}{c_{W}^{2}}\left[O_{j i}^{\prime \prime L} / D_{Z}-N_{i 1}^{*} N_{j 1} /\left(u-m_{\widetilde{e}_{R}}^{2}\right)\right] .
\end{aligned}
$$

Squaring the amplitude and averaging over electron and positron spins, only terms involving $x_{1} x_{1}^{\dagger}$ or $y_{1} y_{1}^{\dagger}$, and $x_{2} x_{2}^{\dagger}$ or $y_{2} y_{2}^{\dagger}$ survive in the massless electron limit. Thus,

$$
\begin{aligned}
\sum_{\lambda_{1}, \lambda_{2}}|\mathcal{M}|^{2}=\sum_{\lambda_{1}, \lambda_{2}} & \left(\left|c_{1}\right|^{2} y_{j}^{\dagger} x_{1}^{\dagger} x_{1} y_{j} x_{i} y_{2} y_{2}^{\dagger} x_{i}^{\dagger}+\left|c_{2}\right|^{2} y_{i}^{\dagger} x_{1}^{\dagger} x_{1} y_{i} x_{j} y_{2} y_{2}^{\dagger} x_{j}^{\dagger}\right. \\
& +\left|c_{3}\right|^{2} x_{i} y_{1} y_{1}^{\dagger} x_{i}^{\dagger} y_{j}^{\dagger} x_{2}^{\dagger} x_{2} y_{j}+\left|c_{4}\right|^{2} x_{j} y_{1} y_{1}^{\dagger} x_{j}^{\dagger} y_{i}^{\dagger} x_{2}^{\dagger} x_{2} y_{i} \\
& +2 \operatorname{Re}\left[c_{1} c_{2}^{*} y_{i}^{\dagger} x_{1}^{\dagger} x_{1} y_{j} x_{j} y_{2} y_{2}^{\dagger} x_{i}^{\dagger}\right] \\
& \left.+2 \operatorname{Re}\left[c_{3} c_{4}^{*} x_{j} y_{1} y_{1}^{\dagger} x_{i}^{\dagger} y_{i}^{\dagger} x_{2}^{\dagger} x_{2} y_{j}\right]\right) \\
=\left|c_{1}\right|^{2} y_{j}^{\dagger} p_{1} \cdot \bar{\sigma} y_{j} x_{i} p_{2} \cdot \sigma x_{i}^{\dagger}+\left|c_{2}\right|^{2} y_{i}^{\dagger} p_{1} \cdot \bar{\sigma} y_{i} x_{j} p_{2} \cdot \sigma x_{j}^{\dagger} & +\left|c_{3}\right|^{2} x_{i} p_{1} \cdot \sigma x_{i}^{\dagger} y_{j}^{\dagger} p_{2} \cdot \bar{\sigma} y_{j}+\left|c_{4}\right|^{2} x_{j} p_{1} \cdot \sigma x_{j}^{\dagger} y_{i}^{\dagger} p_{2} \cdot \bar{\sigma} y_{i} \\
+ & 2 \operatorname{Re}\left[c_{1} c_{2}^{*} y_{i}^{\dagger} p_{1} \cdot \bar{\sigma} y_{j} x_{j} p_{2} \cdot \sigma x_{i}^{\dagger}\right] \\
+ & 2 \operatorname{Re}\left[c_{3} c_{4}^{*} x_{j} p_{1} \cdot \sigma x_{i}^{\dagger} y_{i}^{\dagger} p_{2} \cdot \bar{\sigma} y_{j}\right]
\end{aligned}
$$


after employing the results of eqs. (2.3.3)- 2.3.6).

We now perform the remaining spin sums using eqs. (2.3.3)-(2.3.6) again, obtaining:

$$
\begin{aligned}
\sum_{\lambda_{1}, \lambda_{2}, \lambda_{i}, \lambda_{j}}|\mathcal{M}|^{2}= & \left|c_{1}\right|^{2} \operatorname{Tr}\left[p_{1} \cdot \bar{\sigma} k_{j} \cdot \sigma\right] \operatorname{Tr}\left[p_{2} \cdot \sigma k_{i} \cdot \bar{\sigma}\right] \\
& +\left|c_{2}\right|^{2} \operatorname{Tr}\left[p_{1} \cdot \bar{\sigma} k_{i} \cdot \sigma\right] \operatorname{Tr}\left[p_{2} \cdot \sigma k_{j} \cdot \bar{\sigma}\right] \\
& +\left|c_{3}\right|^{2} \operatorname{Tr}\left[p_{1} \cdot \sigma k_{i} \cdot \bar{\sigma}\right] \operatorname{Tr}\left[p_{2} \cdot \bar{\sigma} k_{j} \cdot \sigma\right] \\
& +\left|c_{4}\right|^{2} \operatorname{Tr}\left[p_{1} \cdot \sigma k_{j} \cdot \bar{\sigma}\right] \operatorname{Tr}\left[p_{2} \cdot \bar{\sigma} k_{i} \cdot \sigma\right] \\
& +2 \operatorname{Re}\left[c_{1} c_{2}^{*}\right] m_{\widetilde{N}_{i}} m_{\widetilde{N}_{j}} \operatorname{Tr}\left[p_{2} \cdot \sigma p_{1} \cdot \bar{\sigma}\right] \\
& +2 \operatorname{Re}\left[c_{3} c_{4}^{*}\right] m_{\widetilde{N}_{i}} m_{\widetilde{N}_{j}} \operatorname{Tr}\left[p_{1} \cdot \sigma p_{2} \cdot \bar{\sigma}\right] .
\end{aligned}
$$

Applying the trace identity of eq. (2.1.43) to this yields

$$
\begin{aligned}
\sum_{\text {spins }}|\mathcal{M}|^{2}= & \left(\left|c_{1}\right|^{2}+\left|c_{4}\right|^{2}\right) 4 p_{1} \cdot k_{j} p_{2} \cdot k_{i}+\left(\left|c_{2}\right|^{2}+\left|c_{3}\right|^{2}\right) 4 p_{1} \cdot k_{i} p_{2} \cdot k_{j} \\
& -4 \operatorname{Re}\left[c_{1} c_{2}^{*}+c_{3} c_{4}^{*}\right] m_{\widetilde{N}_{i}} m_{\widetilde{N}_{j}} p_{1} \cdot p_{2} \\
= & \left(\left|c_{1}\right|^{2}+\left|c_{4}\right|^{2}\right)\left(u-m_{\widetilde{N}_{i}}^{2}\right)\left(u-m_{\widetilde{N}_{j}}^{2}\right) \\
& +\left(\left|c_{2}\right|^{2}+\left|c_{3}\right|^{2}\right)\left(t-m_{\widetilde{N}_{i}}^{2}\right)\left(t-m_{\widetilde{N}_{j}}^{2}\right) \\
& +2 \operatorname{Re}\left[c_{1} c_{2}^{*}+c_{3} c_{4}^{*}\right] m_{\widetilde{N}_{i}} m_{\widetilde{N}_{j}} s .
\end{aligned}
$$

The differential cross-section then follows:

$$
\frac{d \sigma}{d t}=\frac{1}{16 \pi s^{2}}\left(\frac{1}{4} \sum_{\text {spins }}|\mathcal{M}|^{2}\right) .
$$

This agrees with the first complete calculation presented in ref. [23]. For the case of pure photino pair production, i.e. $N_{i 1} \rightarrow c_{W} \delta_{i 1}$ and $N_{i 2} \rightarrow s_{W} \delta_{i 1}$ and for degenerate selectron masses this also agrees with eq. (E9) of the erratum of [1].

Defining $\cos \theta=\hat{\boldsymbol{p}}_{\mathbf{1}} \cdot \hat{\boldsymbol{k}}_{\boldsymbol{i}}$ (the cosine of the angle between the initial state electron and one of the neutralinos in the center-of-momentum frame), the Mandelstam variables $t, u$ are given by

$$
\begin{aligned}
t & =\frac{1}{2}\left[m_{\widetilde{N}_{i}}^{2}+m_{\widetilde{N}_{j}}^{2}-s+\lambda^{1 / 2}\left(s, m_{\widetilde{N}_{i}}^{2}, m_{\widetilde{N}_{j}}^{2}\right) \cos \theta\right], \\
u & =\frac{1}{2}\left[m_{\widetilde{N}_{i}}^{2}+m_{\widetilde{N}_{j}}^{2}-s-\lambda^{1 / 2}\left(s, m_{\widetilde{N}_{i}}^{2}, m_{\widetilde{N}_{j}}^{2}\right) \cos \theta\right],
\end{aligned}
$$

where the triangle function $\lambda^{1 / 2}$ is defined in eq. (5.1.13). Taking into account the identical fermions in the final state when $i=j$, the total cross-section is

$$
\sigma=\frac{1}{1+\delta_{i j}} \int_{t_{-}}^{t_{+}} \frac{d \sigma}{d t} d t
$$

where $t_{-}$and $t_{+}$are obtained by inserting $\cos \theta=\mp 1$ in eq. (5.7.20), respectively.

5.8. $e^{-} e^{+} \rightarrow \widetilde{C}_{i}^{-} \widetilde{C}_{j}^{+}$

Next we consider the pair production of charginos in electron-positron collisions. The $s$-channel Feynman diagrams are shown in Fig. 5.8.1, where we have also introduced 

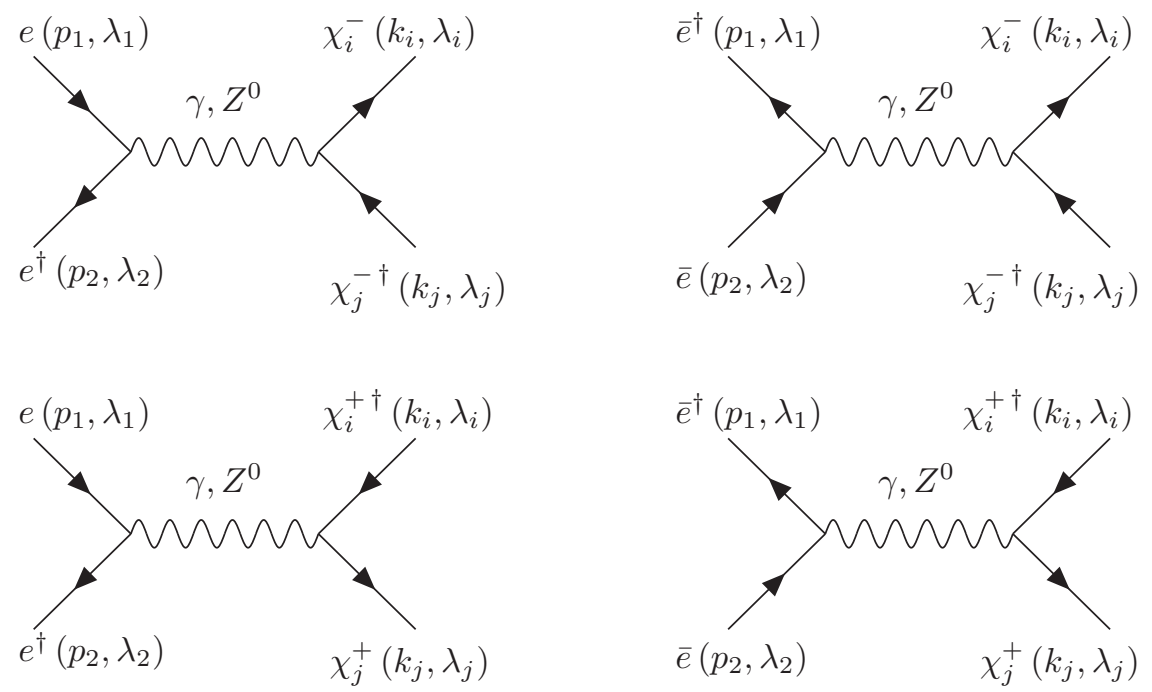

Fig. 5.8.1: Feynman diagrams for $e^{-} e^{+} \rightarrow \widetilde{C}_{i}^{-} \widetilde{C}_{j}^{+}$via $s$-channel $\gamma$ and $Z^{0}$ exchange.

the notation for the fermion momenta and polarizations. The Mandelstam variables are given by

$$
\begin{aligned}
& s=-2 p_{1} \cdot p_{2}=m_{\widetilde{C}_{i}}^{2}+m_{\widetilde{C}_{j}}^{2}-2 k_{i} \cdot k_{j}, \\
& t=m_{\widetilde{C}_{i}}^{2}+2 p_{1} \cdot k_{i}=m_{\widetilde{C}_{j}}^{2}+2 p_{2} \cdot k_{j}, \\
& u=m_{\widetilde{C}_{i}}^{2}+2 p_{2} \cdot k_{i}=m_{\widetilde{C}_{j}}^{2}+2 p_{1} \cdot k_{j} .
\end{aligned}
$$

The negatively charged chargino carries momentum and polarization $\left(k_{i}, \lambda_{i}\right)$, while the positively charged one carries $\left(k_{j}, \lambda_{j}\right)$.

Using the first four Feynman rules of Figure 3.1 and the first four of Figure 4.2, the sum of the photon-exchange diagrams is given by:

$$
i \mathcal{M}_{\gamma}=\frac{i g^{\mu \nu}}{s}\left(i e x_{1} \sigma_{\mu} y_{2}^{\dagger}+i e y_{1}^{\dagger} \bar{\sigma}_{\mu} x_{2}\right)\left(-i e \delta_{i j} y_{i} \sigma_{\nu} x_{j}^{\dagger}-i e \delta_{i j} x_{i}^{\dagger} \bar{\sigma}_{\nu} y_{j}\right)
$$

and the $Z$-exchange diagrams yield:

$$
\begin{gathered}
i \mathcal{M}_{Z}=\frac{i g^{\mu \nu}}{D_{Z}}\left[-\frac{i g}{c_{W}}\left(s_{W}^{2}-\frac{1}{2}\right) x_{1} \sigma_{\mu} y_{2}^{\dagger}-\frac{i g s_{W}^{2}}{c_{W}} y_{1}^{\dagger} \bar{\sigma}_{\mu} x_{2}\right] \\
{\left[\frac{i g}{c_{W}} O_{j i}^{\prime L} y_{i} \sigma_{\nu} x_{j}^{\dagger}+\frac{i g}{c_{W}} O_{j i}^{\prime R} x_{i}^{\dagger} \bar{\sigma}_{\nu} y_{j}\right],}
\end{gathered}
$$

where $D_{Z} \equiv s-m_{Z}^{2}+i \Gamma_{Z} m_{Z}$. The $t$-channel Feynman diagram via sneutrino exchange is shown in Fig. 5.8.2. Applying the eighth rule of Fig. 4.6 and its conjugate with arrows reversed, we find:

$$
i \mathcal{M}_{\widetilde{\nu}_{e}}=(-1) \frac{i}{t-m_{\widetilde{\nu}_{e}}^{2}}\left(-i g V_{i 1}^{*} x_{1} y_{i}\right)\left(-i g V_{j 1} y_{2}^{\dagger} x_{j}^{\dagger}\right)
$$




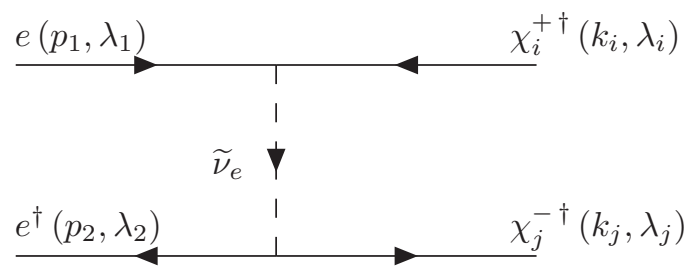

Fig. 5.8.2: The Feynman diagram for $e^{-} e^{+} \rightarrow \widetilde{C}_{i}^{-} \widetilde{C}_{j}^{+}$via the $t$-channel exchange of a sneutrino.

The Fermi-Dirac factor $(-1)$ in this equation arises because the spinors appear in an order which is an odd permutation of the order used in all of the $s$-channel diagram results.

One can now apply the Fierz transformation identities eqs. (2.1.55)-(2.1.57) to eqs. (5.8.4) and (5.8.5) to remove the $\sigma$ and $\bar{\sigma}$ matrices. The result can be combined with the $t$-channel contribution to obtain a total matrix element $\mathcal{M}$ with exactly the same form as eq. (5.7.9), but now with:

$$
\begin{aligned}
& c_{1}=2 \frac{e^{2} \delta_{i j}}{s}-\frac{g^{2}}{c_{W}^{2} D_{Z}}\left(1-2 s_{W}^{2}\right) O_{j i}^{\prime R}, \\
& c_{2}=\frac{2 e^{2} \delta_{i j}}{s}-\frac{g^{2}}{c_{W}^{2} D_{Z}}\left(1-2 s_{W}^{2}\right) O_{j i}^{\prime L}+\frac{g^{2} V_{i 1}^{*} V_{j 1}}{t-m_{\widetilde{\nu}_{e}}^{2}}, \\
& c_{3}=\frac{2 e^{2} \delta_{i j}}{s}+\frac{2 g^{2} s_{W}^{2}}{c_{W}^{2} D_{Z}} O_{j i}^{\prime R}, \\
& c_{4}=\frac{2 e^{2} \delta_{i j}}{s}+\frac{2 g^{2} s_{W}^{2}}{c_{W}^{2} D_{Z}} O_{j i}^{L} .
\end{aligned}
$$

The rest of this calculation is identical in form to eqs. (5.7.9)-(5.7.18), so that the result is:

$$
\begin{aligned}
\sum_{\text {spins }}|\mathcal{M}|^{2}= & \left(\left|c_{1}\right|^{2}+\left|c_{4}\right|^{2}\right)\left(u-m_{\widetilde{C}_{i}}^{2}\right)\left(u-m_{\widetilde{C}_{j}}^{2}\right) \\
+ & \left(\left|c_{2}\right|^{2}+\left|c_{3}\right|^{2}\right)\left(t-m_{\widetilde{C}_{i}}^{2}\right)\left(t-m_{\widetilde{C}_{j}}^{2}\right) \\
& +2 \operatorname{Re}\left[c_{1} c_{2}^{*}+c_{3} c_{4}^{*}\right] m_{\widetilde{C}_{i}} m_{\widetilde{C}_{j}} s .
\end{aligned}
$$

The differential cross-section then follows:

$$
\frac{d \sigma}{d t}=\frac{1}{16 \pi s^{2}}\left(\frac{1}{4} \sum_{\text {spins }}|\mathcal{M}|^{2}\right) .
$$

As in the previous subsection, we define $\cos \theta=\hat{\boldsymbol{p}}_{\mathbf{1}} \cdot \hat{\boldsymbol{k}}_{\boldsymbol{i}}$ (where $\theta$ is the angle between the initial state electron and $\widetilde{C}_{i}^{-}$in the center-of-momentum frame). The Mandelstam variables $t, u$ are given by

$$
\begin{aligned}
& t=\frac{1}{2}\left[m_{\widetilde{C}_{i}}^{2}+m_{\widetilde{C}_{j}}^{2}-s+\lambda^{1 / 2}\left(s, m_{\widetilde{C}_{i}}^{2}, m_{\widetilde{C}_{j}}^{2}\right) \cos \theta\right], \\
& u=\frac{1}{2}\left[m_{\widetilde{C}_{i}}^{2}+m_{\widetilde{C}_{j}}^{2}-s-\lambda^{1 / 2}\left(s, m_{\widetilde{C}_{i}}^{2}, m_{\widetilde{C}_{j}}^{2}\right) \cos \theta\right] .
\end{aligned}
$$



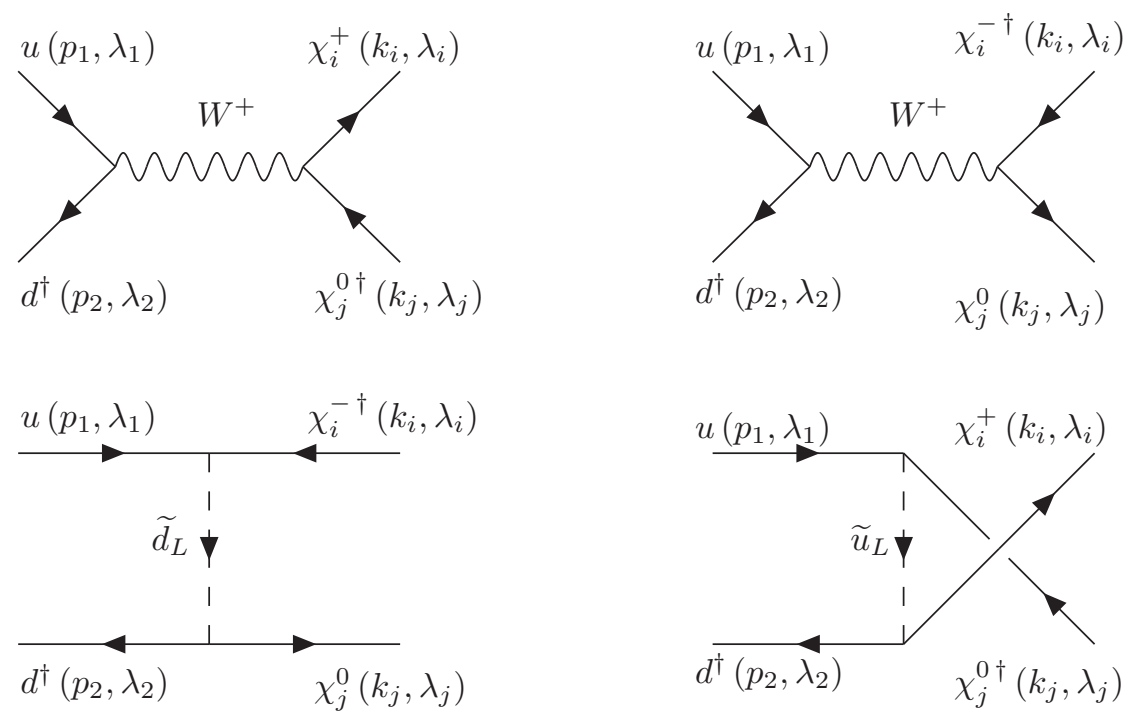

Fig. 5.9.1: The four tree-level Feynman diagrams for $u \bar{d} \rightarrow \widetilde{C}_{i}^{+} \widetilde{N}_{j}$.

The total cross-section can now be computed as

$$
\sigma=\int_{t_{-}}^{t_{+}} \frac{d \sigma}{d t} d t
$$

where $t_{-}$and $t_{+}$are obtained with $\cos \theta=-1$ and +1 in eq. (5.8.13), respectively. This agrees with the original first complete calculation in ref. 24. An extended calculation for the production of polarized charginos is given in ref. [25].

\section{9. $u \bar{d} \rightarrow \widetilde{C}_{i}^{+} \widetilde{N}_{j}$}

Next we consider the associated production of a chargino and a neutralino in quark, antiquark collisions. The leading order Feynman diagrams are shown in Fig. 5.9.1, where we have also defined the momenta and the helicities. The corresponding Mandelstam variables are

$$
\begin{aligned}
& s=-2 p_{1} \cdot p_{2}=m_{\widetilde{C}_{i}}^{2}+m_{\widetilde{N}_{j}}^{2}-2 k_{i} \cdot k_{j}, \\
& t=m_{\widetilde{C}_{i}}^{2}+2 p_{1} \cdot k_{i}=m_{\widetilde{N}_{j}}^{2}+2 p_{2} \cdot k_{j}, \\
& u=m_{\widetilde{C}_{i}}^{2}+2 p_{2} \cdot k_{i}=m_{\widetilde{N}_{j}}^{2}+2 p_{1} \cdot k_{j} .
\end{aligned}
$$

The matrix elements for the $s$-channel diagrams are obtained by applying the fifth Feynman rule of Figure 3.1 and the last two of Figure 4.2;

$$
i \mathcal{M}_{s}=\frac{i g^{\mu \nu}}{s-m_{W}^{2}}\left(-\frac{i g}{\sqrt{2}} x_{1} \sigma_{\mu} y_{2}^{\dagger}\right)\left(-i g O_{j i}^{L *} x_{i}^{\dagger} \bar{\sigma}_{\nu} y_{j}-i g O_{j i}^{R *} y_{i} \sigma_{\nu} x_{j}^{\dagger}\right)
$$

The external spinors are denoted by $x_{1} \equiv x\left(\overrightarrow{\boldsymbol{p}}_{1}, \lambda_{1}\right), y_{2}^{\dagger} \equiv y^{\dagger}\left(\overrightarrow{\boldsymbol{p}}_{2}, \lambda_{2}\right), x_{i}^{\dagger} \equiv x^{\dagger}\left(\overrightarrow{\boldsymbol{k}}_{i}, \lambda_{i}\right)$, $y_{j} \equiv y\left(\overrightarrow{\boldsymbol{k}}_{j}, \lambda_{j}\right)$, etc. The matrix elements for the $t$ and $u$ graphs follow from the first rule 
of Figure 4.7 and the first two of Figure 4.6.

$$
\begin{aligned}
i \mathcal{M}_{t} & =(-1) \frac{i}{t-m_{\widetilde{d}_{L}}^{2}}\left(-i g U_{i 1}^{*}\right)\left(\frac{i g}{\sqrt{2}}\left[N_{j 2}-\frac{s_{W}}{3 c_{W}} N_{j 1}\right]\right) x_{1} y_{i} y_{2}^{\dagger} x_{j}^{\dagger}, \\
i \mathcal{M}_{u} & =\frac{i}{u-m_{\widetilde{u}_{L}}^{2}}\left(-i g V_{i 1}\right)\left(\frac{i g}{\sqrt{2}}\left[-N_{j 2}^{*}-\frac{s_{W}}{3 c_{W}} N_{j 1}^{*}\right]\right) x_{1} y_{j} y_{2}^{\dagger} x_{i}^{\dagger} .
\end{aligned}
$$

The first factor of $(-1)$ in eq. (5.9.5) is required because the order of the spinors $(1, i, 2, j)$ is in an odd permutation of the order $(1,2, i, j)$ used in the $s$-channel and $u$-channel results.

Now we can use the Fierz relations eqs. (2.1.55) and (2.1.57) to rewrite the $s$-channel amplitude in a form without $\sigma$ or $\bar{\sigma}$ matrices. Combining the result with the $t$-channel and $u$-channel contributions yields a total $\mathcal{M}$ with exactly the same form as eq. (5.7.9), but now with

$$
\begin{aligned}
& c_{1}=-\sqrt{2} g^{2}\left[\frac{O_{j i}^{L *}}{s-m_{W}^{2}}+\left(\frac{1}{2} N_{j 2}^{*}+\frac{s_{W}}{6 c_{W}} N_{j 1}^{*}\right) \frac{V_{i 1}}{u-m_{\widetilde{u}_{L}}}\right], \\
& c_{2}=-\sqrt{2} g^{2}\left[\frac{O_{j i}^{R *}}{s-m_{W}^{2}}+\left(\frac{1}{2} N_{j 2}^{*}-\frac{s_{W}}{6 c_{W}} N_{j 1}^{*}\right) \frac{U_{i 1}^{*}}{t-m_{\widetilde{d}_{L}}}\right], \\
& c_{3}=c_{4}=0 .
\end{aligned}
$$

The rest of this calculation is identical in form to that of eqs. (5.7.9)-(5.7.18), leading to:

$$
\begin{aligned}
\sum_{\text {spins }}|\mathcal{M}|^{2}= & \left|c_{1}\right|^{2}\left(u-m_{\widetilde{C}_{i}}^{2}\right)\left(u-m_{\widetilde{N}_{j}}^{2}\right)+\left|c_{2}\right|^{2}\left(t-m_{\widetilde{C}_{i}}^{2}\right)\left(t-m_{\widetilde{N}_{j}}^{2}\right) \\
& +2 \operatorname{Re}\left[c_{1} c_{2}^{*}\right] m_{\widetilde{C}_{i}} m_{\widetilde{N}_{j}} s .
\end{aligned}
$$

From this, one obtains:

$$
\frac{d \sigma}{d t}=\frac{1}{16 \pi s^{2}}\left(\frac{1}{3 \cdot 4} \sum_{\text {spins }}|\mathcal{M}|^{2}\right),
$$

where we have included a factor of $1 / 3$ from the color average for the incoming quarks. As in the previous two subsections, eq. (5.9.11) can be expressed in terms of the angle between the $u$ quark and the chargino in the center-of-momentum frame, using

$$
\begin{aligned}
& t=\frac{1}{2}\left[m_{\widetilde{C}_{i}}^{2}+m_{\widetilde{N}_{j}}^{2}-s+\lambda^{1 / 2}\left(s, m_{\widetilde{C}_{i}}^{2}, m_{\widetilde{N}_{j}}^{2}\right) \cos \theta\right], \\
& u=\frac{1}{2}\left[m_{\widetilde{C}_{i}}^{2}+m_{\widetilde{N}_{j}}^{2}-s-\lambda^{1 / 2}\left(s, m_{\widetilde{C}_{i}}^{2}, m_{\widetilde{N}_{j}}^{2}\right) \cos \theta\right] .
\end{aligned}
$$

This process occurs in proton-antiproton and proton-proton collisions, where $\sqrt{s}$ is not fixed, and the angle $\theta$ is different than the lab frame angle. The observable crosssection depends crucially on experimental cuts. The result in eq. (5.9.11) agrees with the computation in ref. [26]. 

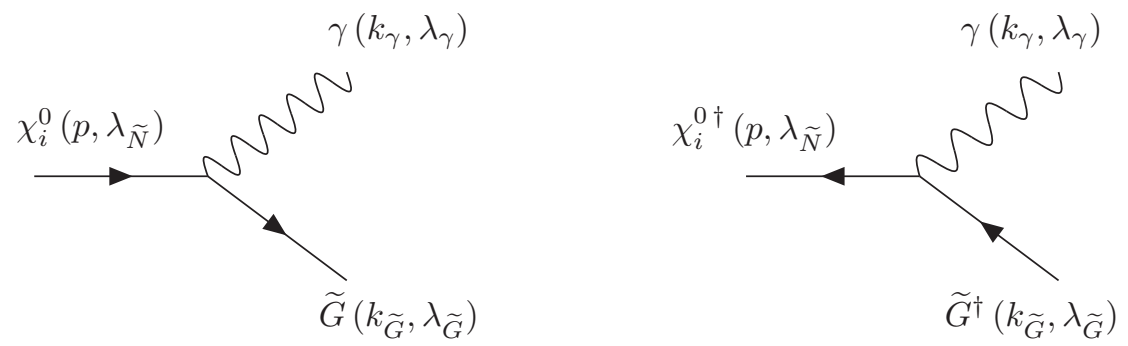

Fig. 5.10.1: The two Feynman diagrams for $\widetilde{N}_{i} \rightarrow \gamma \widetilde{G}$ in supersymmetric models with a light Goldstino.

\subsection{Neutralino decay to photon and Goldstino: $\widetilde{N}_{i} \rightarrow \gamma \widetilde{G}$}

The Goldstino $\widetilde{G}$ is a massless Weyl fermion that couples to the neutralino and photon fields according to a non-renormalizable Lagrangian term [27]:

$$
\mathscr{L}=\frac{a_{i}}{2}\left(\chi_{i}^{0} \sigma^{\mu} \bar{\sigma}^{\rho} \sigma^{\nu} \partial_{\mu} \widetilde{G}^{\dagger}\right)\left(\partial_{\nu} A_{\rho}-\partial_{\rho} A_{\nu}\right)+\text { h.c. }
$$

Here $\chi_{i}^{0}$ is the left-handed 2-component fermion field that corresponds to the neutralino $\widetilde{N}_{i}$ particle, $\widetilde{G}$ is the 2-component fermion field corresponding to the (nearly) massless Goldstino, and the effective coupling is

$$
a_{i} \equiv \frac{1}{\sqrt{2}\langle F\rangle}\left(N_{i 1}^{*} \cos \theta_{W}+N_{i 2}^{*} \sin \theta_{W}\right)
$$

where $N_{i j}$ the mixing matrix for the neutralinos [see eq. (4.3)], and $\langle F\rangle$ is the $F$-term expectation value associated with supersymmetry breaking. Therefore $\widetilde{N}_{i}$ can decay to $\gamma$ plus $\widetilde{G}$ through the diagrams shown in Fig. 5.10.1, with amplitudes:

$$
\begin{aligned}
& i \mathcal{M}_{1}=-i \frac{a_{i}}{2} x_{\widetilde{N}} k_{\widetilde{G}} \cdot \sigma\left(\varepsilon^{*} \cdot \bar{\sigma} k_{\gamma} \cdot \sigma-k_{\gamma} \cdot \bar{\sigma} \varepsilon^{*} \cdot \sigma\right) x_{\widetilde{G}}^{\dagger}, \\
& i \mathcal{M}_{2}=i \frac{a_{i}^{*}}{2} y_{\widetilde{N}}^{\dagger} k_{\widetilde{G}} \cdot \bar{\sigma}\left(\varepsilon^{*} \cdot \sigma k_{\gamma} \cdot \bar{\sigma}-k_{\gamma} \cdot \sigma \varepsilon^{*} \cdot \bar{\sigma}\right) y_{\widetilde{G}} .
\end{aligned}
$$

Here $x_{\widetilde{N}} \equiv x\left(\overrightarrow{\boldsymbol{p}}, \lambda_{\widetilde{N}}\right), y_{\widetilde{N}}^{\dagger} \equiv y^{\dagger}\left(\overrightarrow{\boldsymbol{p}}, \lambda_{\widetilde{N}}\right)$, and $x_{\widetilde{G}}^{\dagger} \equiv x^{\dagger}\left(\overrightarrow{\boldsymbol{k}}_{\widetilde{G}}, \lambda_{\widetilde{G}}\right), y_{\widetilde{G}} \equiv y\left(\overrightarrow{\boldsymbol{k}}_{\widetilde{G}}, \lambda_{\widetilde{G}}\right)$, and $\varepsilon^{*}=\varepsilon^{*}\left(\overrightarrow{\boldsymbol{k}}_{\gamma}, \lambda_{\gamma}\right)$ are the external wave function factors for the neutralino, Goldstino, and photon, respectively.

Using the on-shell condition $k_{\gamma} \cdot \varepsilon^{*}=0$, we have $k_{\gamma} \cdot \sigma \varepsilon^{*} \cdot \bar{\sigma}=-\varepsilon^{*} \cdot \sigma k_{\gamma} \cdot \bar{\sigma}$ and $k_{\gamma} \cdot \bar{\sigma} \varepsilon^{*} \cdot \sigma=$ $-\varepsilon^{*} \cdot \bar{\sigma} k_{\gamma} \cdot \sigma$ from eqs. (2.1.38) and (2.1.39). So we can rewrite the total amplitude as

$$
\mathcal{M}=\mathcal{M}_{1}+\mathcal{M}_{2}=x_{\widetilde{N}} A x_{\widetilde{G}}^{\dagger}+y_{\widetilde{N}}^{\dagger} B y_{\widetilde{G}}
$$

where

$$
\begin{aligned}
& A=-a_{i} k_{\widetilde{G}} \cdot \sigma \varepsilon^{*} \cdot \bar{\sigma} k_{\gamma} \cdot \sigma, \\
& B=a_{i}^{*} k_{\widetilde{G}} \cdot \bar{\sigma} \varepsilon^{*} \cdot \sigma k_{\gamma} \cdot \bar{\sigma} .
\end{aligned}
$$

The complex square of the matrix element is therefore

$$
\begin{aligned}
|\mathcal{M}|^{2}= & x_{\widetilde{N}} A x_{\widetilde{G}}^{\dagger} x_{\widetilde{G}} \hat{A} x_{\widetilde{N}}^{\dagger}+y_{\widetilde{N}}^{\dagger} B y_{\widetilde{G}} y_{\widetilde{G}}^{\dagger} \hat{B} y_{\widetilde{N}} \\
& +x_{\widetilde{N}} A x_{\widetilde{G}}^{\dagger} y_{\widetilde{G}}^{\dagger} \hat{B} y_{\widetilde{N}}+y_{\widetilde{N}}^{\dagger} B y_{\widetilde{G}} x_{\widetilde{G}} \hat{A} x_{\widetilde{N}}^{\dagger},
\end{aligned}
$$


where $\hat{A}$ and $\hat{B}$ are obtained from $A$ and $B$ by reversing the order of the $\sigma$ and $\bar{\sigma}$ matrices and taking the complex conjugates of $a_{i}$ and $\varepsilon$ [cf. eq. (2.5.2) and the associated text].

Summing over the Goldstino spins using eqs. (2.3.3)-(2.3.6) now yields:

$$
\sum_{\lambda_{\widetilde{G}}}|\mathcal{M}|^{2}=-x_{\widetilde{N}} A k_{\widetilde{G}} \cdot \bar{\sigma} \hat{A} x_{\widetilde{N}}^{\dagger}-y_{\widetilde{N}}^{\dagger} B k_{\widetilde{G}} \cdot \sigma \hat{B} y_{\widetilde{N}}
$$

(The $A, \hat{B}$ and $\hat{A}, B$ cross terms vanish because of $m_{\widetilde{G}}=0$.) Averaging over the neutralino spins using eqs. (2.3.3) and (2.3.4), we find

$$
\begin{aligned}
\frac{1}{2} \sum_{\lambda_{\widetilde{N}}, \lambda_{\widetilde{G}}}|\mathcal{M}|^{2}= & \frac{1}{2} \operatorname{Tr}\left[A k_{\widetilde{G}} \cdot \bar{\sigma} \hat{A} p \cdot \bar{\sigma}\right]+\frac{1}{2} \operatorname{Tr}\left[B k_{\widetilde{G}} \cdot \sigma \hat{B} p \cdot \sigma\right] \\
= & \frac{1}{2}\left|a_{i}\right|^{2} \operatorname{Tr}\left[\varepsilon^{*} \cdot \bar{\sigma} k_{\gamma} \cdot \sigma k_{\widetilde{G}} \cdot \bar{\sigma} k_{\gamma} \cdot \sigma \varepsilon \cdot \bar{\sigma} k_{\widetilde{G}} \cdot \sigma p \cdot \bar{\sigma} k_{\widetilde{G}} \cdot \sigma\right] \\
& +(\sigma \leftrightarrow \bar{\sigma}) .
\end{aligned}
$$

We now use

$$
\begin{aligned}
k_{\gamma} \cdot \sigma k_{\widetilde{G}} \cdot \bar{\sigma} k_{\gamma} \cdot \sigma & =-2 k_{\widetilde{G}} \cdot k_{\gamma} k_{\gamma} \cdot \sigma, \\
k_{\widetilde{G}} \cdot \sigma p \cdot \bar{\sigma} k_{\widetilde{G}} \cdot \sigma & =-2 k_{\widetilde{G}} \cdot p k_{\widetilde{G}} \cdot \sigma,
\end{aligned}
$$

which follow from eq. (2.1.40), and the corresponding identities with $\sigma \leftrightarrow \bar{\sigma}$, to obtain:

$$
\begin{aligned}
\frac{1}{2} \sum_{\lambda_{\widetilde{N}}, \lambda_{\widetilde{G}}}|\mathcal{M}|^{2}= & 2\left|a_{i}\right|^{2}\left(k_{\widetilde{G}} \cdot k_{\gamma}\right)\left(k_{\widetilde{G}} \cdot p\right) \operatorname{Tr}\left[\varepsilon^{*} \cdot \bar{\sigma} k_{\gamma} \cdot \sigma \varepsilon \cdot \bar{\sigma} k_{\widetilde{G}} \cdot \sigma\right] \\
& +(\sigma \leftrightarrow \bar{\sigma}) .
\end{aligned}
$$

Applying the photon spin-sum identity

$$
\sum_{\lambda_{\gamma}} \varepsilon^{\mu} \varepsilon^{\nu *}=g^{\mu \nu},
$$

and the trace identities eq. (2.1.44) and (2.1.45), we get

$$
\frac{1}{2} \sum_{\lambda_{\gamma}, \lambda_{\widetilde{N}}, \lambda_{\widetilde{G}}}|\mathcal{M}|^{2}=-16\left|a_{i}\right|^{2}\left(k_{\widetilde{G}} \cdot k_{\gamma}\right)^{2}\left(k_{\widetilde{G}} \cdot p\right)=2\left|a_{i}\right|^{2} m_{\widetilde{N}_{i}}^{6} .
$$

So, the decay rate is [28, 29]:

$$
\begin{aligned}
\Gamma\left(\widetilde{N}_{i} \rightarrow \gamma \widetilde{G}\right) & =\frac{1}{16 \pi m_{\widetilde{N}_{i}}}\left(\frac{1}{2} \sum_{\lambda_{\gamma}, \lambda_{\widetilde{N}}, \lambda_{\widetilde{G}}}|\mathcal{M}|^{2}\right) \\
& =\left|N_{i 1} \cos \theta_{W}+N_{i 2} \sin \theta_{W}\right|^{2} \frac{m_{\widetilde{N}_{i}}^{5}}{16 \pi|\langle F\rangle|^{2}} .
\end{aligned}
$$

\subsection{Gluino pair production from gluon fusion: $g g \rightarrow \widetilde{g} \widetilde{g}$}

In this subsection we will compute the cross-section for the process $g g \rightarrow \widetilde{g} \widetilde{g}$. The relevant Feynman diagrams are shown in Fig. 5.11.1. The initial state gluons have $S U(3)_{c}$ adjoint representation indices $a$ and $b$, with momenta $p_{1}$ and $p_{2}$ and polarization vectors $\varepsilon_{1}^{\mu}=\varepsilon^{\mu}\left(\overrightarrow{\boldsymbol{p}}_{1}, \lambda_{1}\right)$ and $\varepsilon_{2}^{\mu}=\varepsilon^{\mu}\left(\overrightarrow{\boldsymbol{p}}_{2}, \lambda_{2}\right)$, respectively. The final state gluinos carry adjoint 

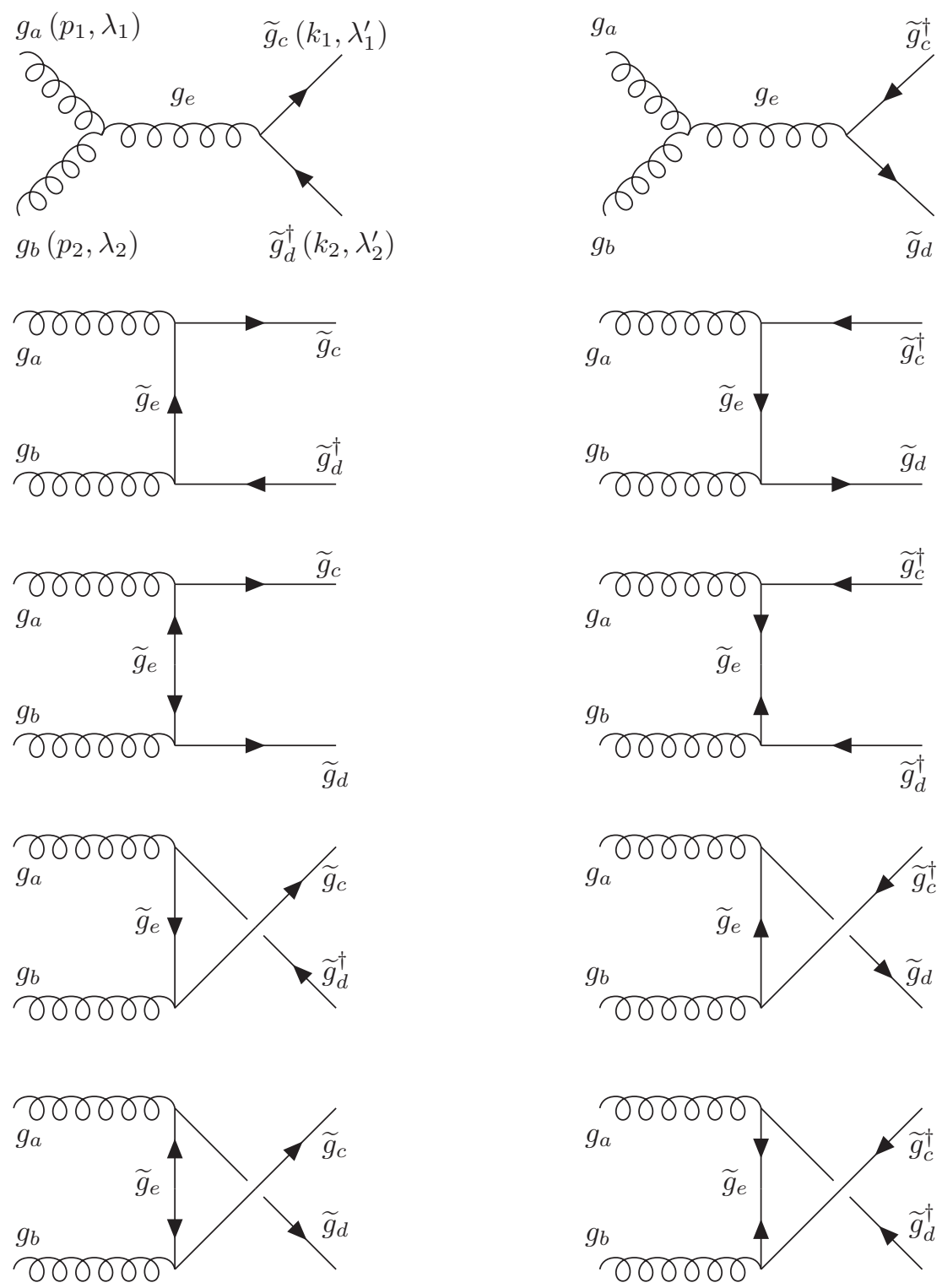

Fig. 5.11.1: The ten Feynman diagrams for $g g \rightarrow \widetilde{g} \widetilde{g}$. The momentum and spin polarization assignments are indicated on the first diagram.

representation indices $c$ and $d$, with momenta $k_{1}$ and $k_{2}$ and wave function spinors $x_{1}^{\dagger}=x^{\dagger}\left(\overrightarrow{\boldsymbol{k}}_{1}, \lambda_{1}^{\prime}\right)$ or $y_{1}=y\left(\overrightarrow{\boldsymbol{k}}_{1}, \lambda_{1}^{\prime}\right)$ and $x_{2}^{\dagger}=x^{\dagger}\left(\overrightarrow{\boldsymbol{k}}_{2}, \lambda_{2}^{\prime}\right)$ or $y_{2}=y\left(\overrightarrow{\boldsymbol{k}}_{2}, \lambda_{2}^{\prime}\right)$, respectively.

The Feynman rule for the gluon coupling to gluinos in the supersymmetric extension of QCD was given in Figure 4.1. For the two s-channel amplitudes, we obtain:

$$
\begin{aligned}
i \mathcal{M}_{s}= & \left(-g_{3} f^{a b e}\left[g_{\mu \nu}\left(p_{1}-p_{2}\right)_{\rho}+g_{\nu \rho}\left(p_{1}+2 p_{2}\right)_{\mu}-g_{\mu \rho}\left(2 p_{1}+p_{2}\right)_{\nu}\right]\right) \\
& \left(\frac{i g^{\rho \kappa}}{s}\right) \varepsilon_{1}^{\mu} \varepsilon_{2}^{\nu}\left[\left(g_{3} f^{c d e}\right) x_{1}^{\dagger} \bar{\sigma}_{\kappa} y_{2}+\left(-g_{3} f^{d c e}\right) y_{1} \sigma_{\kappa} x_{2}^{\dagger}\right] .
\end{aligned}
$$

The first factor is the Feynman rule for the three-gluon interaction of standard QCD, 
and the second factor is the gluon propagator. The next four ( $t$-channel) diagrams have a total amplitude:

$$
\begin{aligned}
i \mathcal{M}_{t}= & \left(g_{3} f^{c e a} \varepsilon_{1}^{\mu}\right)\left(g_{3} f^{e d b} \varepsilon_{2}^{\nu}\right) x_{1}^{\dagger} \bar{\sigma}_{\mu}\left[\frac{i\left(k_{1}-p_{1}\right) \cdot \sigma}{\left(k_{1}-p_{1}\right)^{2}+m_{\widetilde{g}}^{2}}\right] \bar{\sigma}_{\nu} y_{2} \\
& +\left(-g_{3} f^{e c a} \varepsilon_{1}^{\mu}\right)\left(-g_{3} f^{d e b} \varepsilon_{2}^{\nu}\right) y_{1} \sigma_{\mu}\left[\frac{i\left(k_{1}-p_{1}\right) \cdot \bar{\sigma}}{\left(k_{1}-p_{1}\right)^{2}+m_{\widetilde{g}}^{2}}\right] \sigma_{\nu} x_{2}^{\dagger} \\
& +\left(g_{3} f^{c e a} \varepsilon_{1}^{\mu}\right)\left(-g_{3} f^{d e b} \varepsilon_{2}^{\nu}\right) x_{1}^{\dagger} \bar{\sigma}_{\mu}\left[\frac{-i m_{\widetilde{g}}}{\left(k_{1}-p_{1}\right)^{2}+m_{\widetilde{g}}^{2}}\right] \sigma_{\nu} x_{2}^{\dagger} \\
& +\left(-g_{3} f^{e c a} \varepsilon_{1}^{\mu}\right)\left(g_{3} f^{e d b} \varepsilon_{2}^{\nu}\right) y_{1} \sigma_{\mu}\left[\frac{-i m_{\widetilde{g}}}{\left(k_{1}-p_{1}\right)^{2}+m_{\widetilde{g}}^{2}}\right] \bar{\sigma}_{\nu} y_{2} .
\end{aligned}
$$

Finally, the $u$-channel Feynman diagrams result in:

$$
\begin{aligned}
i \mathcal{M}_{u}= & \left(g_{3} f^{e d a} \varepsilon_{1}^{\mu}\right)\left(g_{3} f^{c e b} \varepsilon_{2}^{\nu}\right) x_{1}^{\dagger} \bar{\sigma}_{\nu}\left[\frac{i\left(k_{1}-p_{2}\right) \cdot \sigma}{\left(k_{1}-p_{2}\right)^{2}+m_{\widetilde{g}}^{2}}\right] \bar{\sigma}_{\mu} y_{2} \\
& +\left(-g_{3} f^{d e a} \varepsilon_{1}^{\mu}\right)\left(-g_{3} f^{e c b} \varepsilon_{2}^{\nu}\right) y_{1} \sigma_{\nu}\left[\frac{i\left(k_{1}-p_{2}\right) \cdot \bar{\sigma}}{\left(k_{1}-p_{2}\right)^{2}+m_{\widetilde{g}}^{2}}\right] \sigma_{\mu} x_{2}^{\dagger} \\
& +\left(-g_{3} f^{d e a} \varepsilon_{1}^{\mu}\right)\left(g_{3} f^{c e b} \varepsilon_{2}^{\nu}\right) x_{1}^{\dagger} \bar{\sigma}_{\nu}\left[\frac{-i m_{\widetilde{g}}}{\left(k_{1}-p_{2}\right)^{2}+m_{\widetilde{g}}^{2}}\right] \sigma_{\mu} x_{2}^{\dagger} \\
& +\left(g_{3} f^{e d a} \varepsilon_{1}^{\mu}\right)\left(-g_{3} f^{e c b} \varepsilon_{2}^{\nu}\right) y_{1} \sigma_{\nu}\left[\frac{-i m_{\widetilde{g}}}{\left(k_{1}-p_{2}\right)^{2}+m_{\widetilde{g}}^{2}}\right] \bar{\sigma}_{\mu} y_{2} .
\end{aligned}
$$

We choose to work with real transverse polarization vectors $\varepsilon_{1}, \varepsilon_{2}$. These vectors must both be orthogonal to the initial state collision axis in the center-of-momentum frame. Hence,

$$
\begin{aligned}
& \varepsilon_{1} \cdot \varepsilon_{1}=\varepsilon_{2} \cdot \varepsilon_{2}=1 \\
& \varepsilon_{1} \cdot p_{1}=\varepsilon_{2} \cdot p_{1}=\varepsilon_{1} \cdot p_{2}=\varepsilon_{2} \cdot p_{2}=0, \\
& \varepsilon_{1} \cdot k_{2}=-\varepsilon_{1} \cdot k_{1} \\
& \varepsilon_{2} \cdot k_{2}=-\varepsilon_{2} \cdot k_{1}
\end{aligned}
$$

for each choice of $\lambda_{1}, \lambda_{2}$. The sums over gluon polarizations are performed using:

$$
\sum_{\lambda_{1}} \varepsilon_{1}^{\mu} \varepsilon_{1}^{\nu}=\sum_{\lambda_{2}} \varepsilon_{2}^{\mu} \varepsilon_{2}^{\nu}=g^{\mu \nu}+2\left(p_{1}^{\mu} p_{2}^{\nu}+p_{2}^{\mu} p_{1}^{\nu}\right) / s
$$

In QCD processes with two or more external gluons, the term $2\left(p_{1}^{\mu} p_{2}^{\nu}+p_{2}^{\mu} p_{1}^{\nu}\right) / s$ in eq. (5.11.8) cannot in general be dropped [30]. This is to be contrasted to the photon polarization sum [cf. eq. (5.10.14)], where this latter term can always be neglected (due to a Ward identity of quantum electrodynamics).

Before taking the complex square of the amplitude, it is convenient to rewrite the last two terms in each of eqs. (5.11.2) and (5.11.3) by using the identities [see eq. (2.3.10)]:

$$
m_{\widetilde{g}} x_{1}^{\dagger}=-y_{1}\left(k_{1} \cdot \sigma\right), \quad m_{\widetilde{g}} y_{1}=-x_{1}^{\dagger}\left(k_{1} \cdot \bar{\sigma}\right) .
$$


Using eqs. (2.1.40) and (2.1.41), the resulting total matrix element is then reduced to a sum of terms that each contain exactly one $\sigma$ or $\bar{\sigma}$ matrix. We define convenient factors:

$$
\begin{aligned}
G_{s} & \equiv g_{3}^{2} f^{a b e} f^{c d e} / s \\
G_{t} & \equiv g_{3}^{2} f^{a c e} f^{b d e} /\left(t-m_{\widetilde{g}}^{2}\right), \\
G_{u} & \equiv g_{3}^{2} f^{a d e} f^{b c e} /\left(u-m_{\widetilde{g}}^{2}\right) .
\end{aligned}
$$

where the usual Mandelstam variables are:

$$
\begin{aligned}
& s=-\left(p_{1}+p_{2}\right)^{2}=-\left(k_{1}+k_{2}\right)^{2}, \\
& t=-\left(k_{1}-p_{1}\right)^{2}=-\left(k_{2}-p_{2}\right)^{2}, \\
& u=-\left(k_{1}-p_{2}\right)^{2}=-\left(k_{2}-p_{1}\right)^{2} .
\end{aligned}
$$

Then the total amplitude is (noting that the gluon polarizations $\varepsilon_{1}, \varepsilon_{2}$ were chosen real):

$$
\mathcal{M}=\mathcal{M}_{s}+\mathcal{M}_{t}+\mathcal{M}_{u}=x_{1}^{\dagger} a \cdot \bar{\sigma} y_{2}+y_{1} a^{*} \cdot \sigma x_{2}^{\dagger},
$$

where

$$
\begin{aligned}
a^{\mu} \equiv & -\left(G_{t}+G_{s}\right) \varepsilon_{1} \cdot \varepsilon_{2} p_{1}^{\mu}-\left(G_{u}-G_{s}\right) \varepsilon_{1} \cdot \varepsilon_{2} p_{2}^{\mu}-2 G_{t} k_{1} \cdot \varepsilon_{1} \varepsilon_{2}^{\mu} \\
& -2 G_{u} k_{1} \cdot \varepsilon_{2} \varepsilon_{1}^{\mu}-i \epsilon^{\mu \nu \rho \kappa} \varepsilon_{1 \nu} \varepsilon_{2 \rho}\left(G_{t} p_{1}-G_{u} p_{2}\right)_{\kappa} .
\end{aligned}
$$

Squaring the amplitude using eqs. (2.1.31) and (2.1.32), we get:

$$
\begin{aligned}
|\mathcal{M}|^{2}= & x_{1}^{\dagger} a \cdot \bar{\sigma} y_{2} y_{2}^{\dagger} a^{*} \cdot \bar{\sigma} x_{1}+y_{1} a^{*} \cdot \sigma x_{2}^{\dagger} x_{2} a \cdot \sigma y_{1}^{\dagger} \\
& +x_{1}^{\dagger} a \cdot \bar{\sigma} y_{2} x_{2} a \cdot \sigma y_{1}^{\dagger}+y_{1} a^{*} \cdot \sigma x_{2}^{\dagger} y_{2}^{\dagger} a^{*} \cdot \bar{\sigma} x_{1} .
\end{aligned}
$$

Summing over the gluino spins using eqs. (2.3.3)-(2.3.6), we find:

$$
\begin{aligned}
\sum_{\lambda_{1}^{\prime}, \lambda_{2}^{\prime}}|\mathcal{M}|^{2}= & \operatorname{Tr}\left[a \cdot \bar{\sigma} k_{2} \cdot \sigma a^{*} \cdot \bar{\sigma} k_{1} \cdot \sigma\right]+\operatorname{Tr}\left[a^{*} \cdot \sigma k_{2} \cdot \bar{\sigma} a \cdot \sigma k_{1} \cdot \bar{\sigma}\right] \\
& -m_{\widetilde{g}}^{2} \operatorname{Tr}[a \cdot \bar{\sigma} a \cdot \sigma]-m_{\widetilde{g}}^{2} \operatorname{Tr}\left[a^{*} \cdot \sigma a^{*} \cdot \bar{\sigma}\right] .
\end{aligned}
$$

Performing the traces with eqs. (2.1.43) $-(2.1 .45)$ then yields:

$$
\begin{aligned}
\sum_{\lambda_{1}^{\prime}, \lambda_{2}^{\prime}}|\mathcal{M}|^{2}= & 8 \operatorname{Re}\left[a \cdot k_{1} a^{*} \cdot k_{2}\right]-4 a \cdot a^{*} k_{1} \cdot k_{2} \\
& -4 i \epsilon^{\mu \nu \rho \kappa} k_{1 \mu} k_{2 \nu} a_{\rho} a_{\kappa}^{*}+4 m_{\widetilde{g}}^{2} \operatorname{Re}\left[a^{2}\right] .
\end{aligned}
$$

Inserting the explicit form for $a^{\mu}$ [eq. (5.11.17)] into the above result:

$$
\begin{aligned}
\sum_{\lambda_{1}^{\prime}, \lambda_{2}^{\prime}}|\mathcal{M}|^{2}= & 2\left(t-m_{\tilde{g}}^{2}\right)\left(u-m_{\tilde{g}}^{2}\right)\left[\left(G_{t}+G_{u}\right)^{2}\right. \\
& \left.+4\left(G_{s}+G_{t}\right)\left(G_{s}-G_{u}\right)\left(\varepsilon_{1} \cdot \varepsilon_{2}\right)^{2}\right]-16\left(G_{t}+G_{u}\right)\left[G_{s}(t-u)\right. \\
& \left.+G_{t}\left(t-m_{\tilde{g}}^{2}\right)+G_{u}\left(u-m_{\tilde{g}}^{2}\right)\right]\left(\varepsilon_{1} \cdot \varepsilon_{2}\right)\left(k_{1} \cdot \varepsilon_{1}\right)\left(k_{1} \cdot \varepsilon_{2}\right) \\
& -32\left(G_{t}+G_{u}\right)^{2}\left(k_{1} \cdot \varepsilon_{1}\right)^{2}\left(k_{1} \cdot \varepsilon_{2}\right)^{2} .
\end{aligned}
$$

The sums over gluon polarizations can be done using eq. (5.11.8), which implies:

$$
\begin{aligned}
& \sum_{\lambda_{1}, \lambda_{2}} 1=4, \quad \sum_{\lambda_{1}, \lambda_{2}}\left(\varepsilon_{1} \cdot \varepsilon_{2}\right)^{2}=2, \\
& \sum_{\lambda_{1}, \lambda_{2}}\left(\varepsilon_{1} \cdot \varepsilon_{2}\right)\left(k_{1} \cdot \varepsilon_{1}\right)\left(k_{1} \cdot \varepsilon_{2}\right)=-m_{\widetilde{g}}^{2}+\left(t-m_{\widetilde{g}}^{2}\right)\left(u-m_{\widetilde{g}}^{2}\right) / s, \\
& \sum_{\lambda_{1}, \lambda_{2}}\left(k_{1} \cdot \varepsilon_{1}\right)^{2}\left(k_{1} \cdot \varepsilon_{2}\right)^{2}=\left(m_{\widetilde{g}}^{2}-\left(t-m_{\widetilde{g}}^{2}\right)\left(u-m_{\widetilde{g}}^{2}\right) / s\right)^{2} .
\end{aligned}
$$


Summing over colors using $f^{a b e} f^{c d e} f^{a b e^{\prime}} f^{c d e^{\prime}}=2 f^{a b e} f^{c d e} f^{a c e^{\prime}} f^{b d e^{\prime}}=N_{c}^{2}\left(N_{c}^{2}-1\right)=72$,

$$
\begin{array}{ll}
\sum_{\text {colors }} G_{s}^{2}=\frac{72 g_{3}^{4}}{s^{2}}, & \sum_{\text {colors }} G_{t}^{2}=\frac{72 g_{3}^{4}}{\left(t-m_{\widetilde{g}}^{2}\right)^{2}}, \\
\sum_{\text {colors }} G_{u}^{2}=\frac{72 g_{3}^{4}}{\left(u-m_{\widetilde{g}}^{2}\right)^{2}}, & \sum_{\text {colors }} G_{s} G_{t}=\frac{36 g_{3}^{4}}{s\left(t-m_{\widetilde{g}}^{2}\right)}, \\
\sum_{\text {colors }} G_{s} G_{u}=-\frac{36 g_{3}^{4}}{s\left(u-m_{\widetilde{g}}^{2}\right)}, & \sum_{\text {colors }} G_{t} G_{u}=\frac{36 g_{3}^{4}}{\left(t-m_{\widetilde{g}}^{2}\right)\left(u-m_{\widetilde{g}}^{2}\right)} .
\end{array}
$$

Putting all the factors together, and averaging over the initial state colors and spins, we have:

$$
\begin{aligned}
\frac{d \sigma}{d t}= & \frac{1}{16 \pi s^{2}}\left(\frac{1}{64} \sum_{\text {colors }} \frac{1}{4} \sum_{\text {spins }}|\mathcal{M}|^{2}\right) \\
= & \frac{9 \pi \alpha_{s}^{2}}{4 s^{4}}\left[2\left(t-m_{\widetilde{g}}^{2}\right)\left(u-m_{\widetilde{g}}^{2}\right)-3 s^{2}-4 m_{\widetilde{g}}^{2} s\right. \\
& \left.+\frac{s^{2}\left(s+2 m_{\widetilde{g}}^{2}\right)^{2}}{\left(t-m_{\widetilde{g}}^{2}\right)\left(u-m_{\widetilde{g}}^{2}\right)}-\frac{4 m_{\widetilde{g}}^{4} s^{4}}{\left(t-m_{\widetilde{g}}^{2}\right)^{2}\left(u-m_{\widetilde{g}}^{2}\right)^{2}}\right],
\end{aligned}
$$

which agrees with the result of refs. [31,32] (after some rearrangement). In the centerof-momentum frame, the Mandelstam variable $t$ is related to the scattering angle $\theta$ between an initial state gluon and a final state gluino by:

$$
t=m_{\widetilde{g}}^{2}+\frac{s}{2}\left(\cos \theta \sqrt{1-4 m_{\widetilde{g}}^{2} / s}-1\right) .
$$

Since the final state has identical particles, the total cross-section can now be obtained by:

$$
\sigma=\frac{1}{2} \int_{t_{-}}^{t_{+}} \frac{d \sigma}{d t} d t
$$

where $t_{ \pm}$are obtained by inserting $\cos \theta= \pm 1$ into eq. (5.11.29).

\section{Conclusion}

The preceding notes have some important omissions; there is nothing in the way of history or proper attribution, no derivations or proofs, and no discussion of anomaly cancellation or other loop diagrams involving fermions. For these and many more details see ref. [12], on which these notes are based.

Even more glaring, of course, is the lack of mention of the current status of the search for supersymmetry, the ostensible subject. There are a couple of reasons for this. First, the experimental progress is so rapid that anything I could write would be obsolete on a time scale of weeks. Second, at this writing (April 2012), there is nothing at all to discuss as far as hints of possible superpartner discovery signals. I think most theorists who have worked on supersymmetry extensively are surprised by this; either we have been wrong all along about supersymmetry at the weak scale, or else we were just too 
optimistic about its early discovery. If indeed there is no supersymmetry to be had within the reach of the LHC, then perhaps there will be some other surprises, hopefully of the kind that nobody has dreamed of yet. That would be a great outcome; muchneeded humility lessons for many of us older folks, and new hard puzzles to be worked out by the young!

Personally, I remain guardedly optimistic about supersymmetry, however. The reason is that the hierarchy problem associated with the smallness of the electroweak scale is still there. The squared mass parameter of the Higgs field is quadratically sensitive, through radiative corrections, to every other larger mass scale to which it couples, directly or indirectly. Note that there are good hints for several such mass scales, besides the Planck scale. The quantization of weak hypercharge, the way that the fermion representations of the Standard Model fit into $S U(5)$ and $S O(10)$ multiplets, and the renormalization group running convergence of gauge couplings all hint at some sort of full or partial unification of forces, the scale of which (if it exists) must be very high to evade proton decay and other bounds. Of course, this might be just a coincidence, and the hierarchy problem definitely should not be viewed as hinging on the existence of unification. Other affirmative, and perhaps stronger, hints of the existence of mass scales far above the electroweak scale include: the presence of neutrino masses, which are most naturally explained with the seesaw mechanism; the puzzle of the origin of baryogenesis, which cannot be explained in the Standard Model alone because of the lack of sufficient CP violation; the solution of the strong $\mathrm{CP}$ problem, which can be explained by axions, but only if the Peccei-Quinn breaking scale is very high; and the existence of dark matter.

There is a hint of a $\sim 125 \mathrm{GeV}$ Higgs boson, which is compatible with LHC-scale supersymmetry (even in its minimal form, if the top squarks are rather heavy or highly mixed). Moreover, a large range of heavier Higgs bosons not compatible with supersymmetry have now been ruled out by the LHC, if they are at all Standard-Model like. The lack of LHC hints for exotic physics at this writing suggests that none of the other theories that have been proposed to address the hierarchy problem seem to be in any better shape than supersymmetry is. So, my best guess is that the superpartners are still out there and will eventually be found at the LHC. Fortunately, we shall see.

\section{Acknowledgments}

I am grateful to Herbi Dreiner and Howie Haber, my collaborators in ref. [12, on which these notes are entirely based. I thank the program co-directors Konstantin Matchev and Tim Tait and the local organizers Tom DeGrand and K.T. Mahanthappa, for the opportunity to lecture at the beautifully organized TASI 2011, as well as the students for their attention and insightful questions. This work was supported in part by the National Science Foundation grant number PHY-1068369.

\section{References}

1. H.E. Haber and G.L. Kane, "The Search for Supersymmetry: Probing Physics Beyond the Standard Model," Phys. Rept. 117, 75 (1985). 
2. M.F. Sohnius, "Introducing Supersymmetry," Phys. Rept. 128, 39 (1985).

3. J. Wess and J. Bagger, Supersymmetry and Supergravity, (Princeton Univ. Press, 1992).

4. S. Weinberg, The Quantum Theory of Fields, Vol. 3: Supersymmetry, (Cambridge University Press, 2000).

5. M. Drees, R. Godbole and P. Roy, Theory and Phenomenology of Sparticles, (World Scientific, 2004).

6. H. Baer and X. Tata, Weak Scale Supersymmetry, (Cambridge University Press, 2006).

7. P. Binetruy, Supersymmetry, (Oxford University Press, 2006).

8. J. Terning, Modern Supersymmetry: Dynamics and Duality (Oxford University Press, 2006).

9. M. Dine, Supersymmetry and String Theory: Beyond the Standard Model (Cambridge University Press, 2007)

10. E. Poppitz and S.P. Trivedi, Ann. Rev. Nucl. Part. Sci. 48, 307 (1998) hep-th/9803107]. Y. Shadmi and Y. Shirman, Rev. Mod. Phys. 72, 25 (2000) hep-th/9907225]. K.A. Intriligator and N. Seiberg, Class. Quant. Grav. 24, S741 (2007) hep-ph/0702069. M. Dine and J.D. Mason, Rept. Prog. Phys. 74, 056201 (2011) [1012.2836 [hep-th]].

11. S.P. Martin, "A supersymmetry primer," hep-ph/9709356 (version 6, December 2011).

12. H.K. Dreiner, H.E. Haber and S.P. Martin, "Two-component spinor techniques and Feynman rules for quantum field theory and supersymmetry," Phys. Rept. 494, 1 (2010) [0812.1594 [hep-ph]].

13. W.A. Bardeen, A.J. Buras, D.W. Duke and T. Muta, Free Phys. Rev. D18 (1978) 3998.

14. W. Siegel, Phys. Lett. 84B (1979) 193; D.M. Capper, D.R.T. Jones and P. van Nieuwenhuizen, Nucl. Phys. B167 (1980) 479.

15. W. Siegel, Phys. Lett. 94B (1980) 37.

16. L.V. Avdeev, Theor. Math. Phys. 58 (1984) 203; L.V. Avdeev and A.A. Vladimirov, Nucl. Phys. B219 (1983) 262.

17. M. Blatter, Helv. Phys. Acta 65 (1992) 1011.

18. See e.g. M.S. Chanowitz, M. Furman and I. Hinchliffe, Nucl. Phys. B159 (1979) 225, and references therein.

19. I. Jack and D. R. T. Jones, "Regularization of supersymmetric theories," hep-ph/9707278.

20. J.F. Gunion, H.E. Haber, G. Kane and S. Dawson, The Higgs Hunter's Guide (Westview Press, Boulder, CO, 2000).

21. J.F. Gunion and H.E. Haber, Phys. Rev. D37 (1988) 2515.

22. J.F. Gunion and H.E. Haber, Nucl. Phys. B272 (1986) 1 [Erratum-ibid. B402 (1993) 567].

23. A. Bartl, H. Fraas and W. Majerotto, Nucl. Phys. B278 (1986) 1.

24. A. Bartl, H. Fraas and W. Majerotto, Z. Phys. C30 (1986) 441.

25. S.Y. Choi, A. Djouadi, H.K. Dreiner, J. Kalinowski and P.M. Zerwas, Eur. Phys. J. C7 (1999) 123 hep-ph/9806279.

26. W. Beenakker, M. Klasen, M. Kramer, T. Plehn, M. Spira and P.M. Zerwas, Phys. Rev. Lett. 83 (1999) 3780 hep-ph/9906298].

27. P. Fayet, Phys. Lett. 70B (1977) 461; 86B (1979) 272; R. Casalbuoni, S. De Curtis, D. Dominici, F. Feruglio and R. Gatto, Phys. Lett. B215 (1988) 313; Phys. Rev. D39 (1989) 2281.

28. N. Cabibbo, G.R. Farrar and L. Maiani, Phys. Lett. 105B (1981) 155; D.R. Stump, M. Wiest and C.P. Yuan, Phys. Rev. D54 (1996) 1936 hep-ph/9601362]; S. Ambrosanio, G.L. Kane, G.D. Kribs, S.P. Martin and S. Mrenna, Phys. Rev. Lett. 76 (1996) 3498 hep-ph/9602239; Phys. Rev. D54 (1996) 5395 hep-ph/9605398].

29. S. Dimopoulos, M. Dine, S. Raby and S.D. Thomas, Phys. Rev. Lett. 76 (1996) 3494 hep-ph/9601367.

30. R. Cutler and D.W. Sivers, Phys. Rev. D17 (1978) 196.

31. P.R. Harrison and C.H. Llewellyn Smith, Nucl. Phys. B213 (1983) 223 [Erratum-ibid. B223 (1983) $542]$.

32. S. Dawson, E. Eichten and C. Quigg, Phys. Rev. D31 (1985) 1581. 